\title{
Modelling the chemical evolution of the Milky Way
}

\section{Francesca Matteucci $^{1}$}

Received: 10 February 2021 / Accepted: 31 May 2021 / Published online: 27 August 2021

(C) The Author(s) 2021. This article is published with open access at Springerlink.com

\begin{abstract}
In this review, I will discuss the comparison between model results and observational data for the Milky Way, the predictive power of such models as well as their limits. Such a comparison, known as Galactic archaeology, allows us to impose constraints on stellar nucleosynthesis and timescales of formation of the various Galactic components (halo, bulge, thick disk and thin disk).
\end{abstract}

Keywords The Galaxy · Chemical evolution · Chemical abundances

\section{Contents}

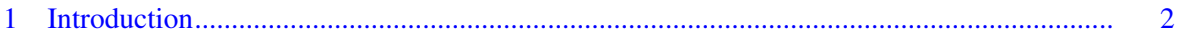

2 Basic ingredients to build chemical models................................................................... 7

2.1 Initial conditions ..............................................................................................

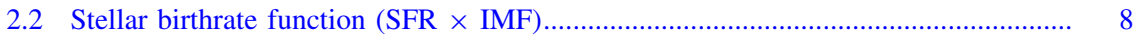

2.2.1 Parametrization of the SFR.................................................................... 8

2.2.2 Parametrization of the IMF............................................................................. 8

2.3 Stellar nucleosynthesis..................................................................................... 9

2.3.1 The yield per stellar generation............................................................ 11

2.4 Gas flows: infall, outflow, radial flows ................................................................. 12

3 Analytical models of chemical evolution....................................................................... 13

3.1 Basic assumptions and solution of the Simple Model ............................................. 13

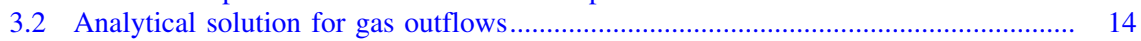

3.3 Analytical solution for gas infall .................................................................... 15

3.4 Analytical solution for gas infall plus outflow .................................................... 15

3.5 Analytical solution for biased galactic outflow plus infall........................................ 15

3.6 More complex analytical solutions .................................................................... 16

4 Numerical chemical evolution models of the Milky Way ............................................... 18

4.1 Basic equations of chemical evolution ................................................................. 19

Francesca Matteucci

francesca.matteucci@inaf.it

1 Department of Physics, Trieste University, Trieste, Italy 
4.2 The supernova rates ....................................................................................... 20

4.2.1 Rate of CC-SNe ............................................................................... 20

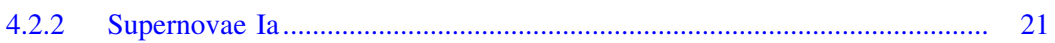

4.2.3 Novae ....................................................................................... 23

4.2.4 Rate of MNS (kilonovae) …............................................................... 24

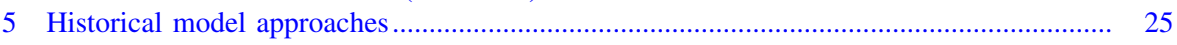

5.1 Serial approach and time delay model ……............................................................. 26

5.2 Parallel approach ................................................................................... 27

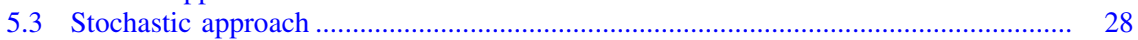

5.4 The classical two-infall model .............................................................................. $\quad 30$

5.4.1 A revised two-infall model and the bimodality in the $[\alpha / \mathrm{Fe}]$ ratios........... 33

5.5 A three-infall model .................................................................................... 34

6 The evolution of heavy elements in halo and disks ...................................................... 34

6.1 Very heavy elements: s- and r-processes ............................................................ 39

6.1.1 s-process elements....................................................................... 39

6.1.2 r-process elements ................................................................ 40

7 Abundance gradients along the thin disk .................................................................. 42

7.1 The $[\alpha / \mathrm{Fe}]$ ratio bimodality at different Galactocentric distances ........................... 43

8 A different paradigm: stellar migration........................................................................ 48

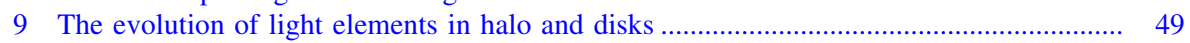

9.1 The chemical evolution of Li.............................................................................. 51

9.2 The chemical evolution of $\mathrm{D}$.................................................................... 53

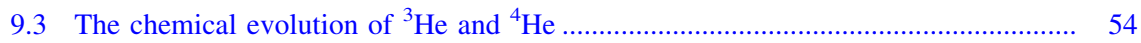

10 The chemical evolution of the Galactic bulge ................................................................ 55

10.1 The evolution of $\alpha$-elements in the bulge ……...................................................... 55

10.2 The evolution of $\mathrm{n}$-capture elements in the bulge ................................................ 58

10.3 Chemical evolution of the Galactic centre ............................................................... 59

11 The chemical evolution of the Galactic halo .................................................................. 60

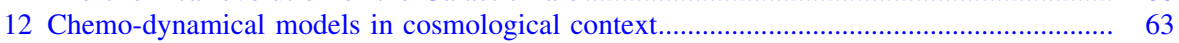

12.1 The Milky Way in a cosmological context ....................................................... 64

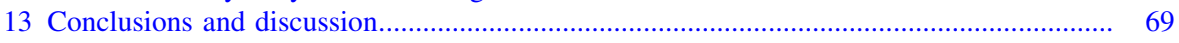

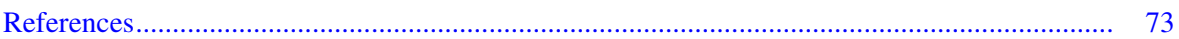

\section{Introduction}

Our Galaxy, the Milky Way, is by far the best studied stellar system. At the present time there is a large flood of data coming from large spectroscopic Galactic surveys, such as Gaia-ESO Public Spectroscopic Survey (GES, Gilmore et al. 2012), the Apache Point Observatory Galactic Evolution Experiment (APOGEE, Majewski et al. 2017) and the GALactic Archaeology with HERMES (GALAH, De Silva et al. 2015), providing data for thousands of stars in all Milky Way stellar components: halo, thick disk, thin disk and bulge. At the same time, a great deal of chemical evolution models, trying to reproduce the observed abundance patterns, have appeared. Galactic chemical evolution tries to explain how the chemical elements have formed and their abundances have evolved in time and distributed in space. It is well known that during the Big Bang only light elements $\left(\mathrm{H}, \mathrm{D},{ }^{3} \mathrm{He}\right.$, ${ }^{4} \mathrm{He},{ }^{7} \mathrm{Li}$ ) were formed, whereas all the elements from ${ }^{12} \mathrm{C}$ to uranium and beyond have been formed inside stars. In particular, chemical evolution models aim at following the evolution of the gas and its chemical composition inside galaxies. 
Such an evolution depends on the star formation history, stellar nucleosynthesis and possible gas flows. The very first seminal paper on chemical evolution is from Schmidt (1963), followed by Lynden-Bell (1975), Pagel and Patchett (1975), and Tinsley (1980). The simplest model for the chemical evolution is called the Simple Model because of its basic (although unrealistic) assumptions, such as the fact that the studied system behaves as a closed-box. The solution of this model can be obtained analytically if the stellar lifetimes are neglected, and provides a relation between the global gas metallicity $Z$ (all the elements heavier than $\mathrm{He}$ ) and the fraction of gas in the studied system. The first pioneering chemical papers from Lynden-Bell (1975) and Pagel and Patchett (1975) discussed how to solve the $G$ dwarf problem, namely, the excess of low metallicity stars, as predicted by the Simple Model, when compared to observations. The G-dwarfs are those in the solar vicinity, a cylinder centered in the Sun with $1 \mathrm{kpc}$ radius, and belong to the thin disk. The most accepted solution to the G-dwarf problem consists in the fact that the solar vicinity should not have evolved as a closed-box, but rather suffered gas flows, in particular gas infall. Tinsley (1980) produced a seminal review paper on galactic chemical evolution which represents the foundation of this field: in that paper, she summarizes the basic ingredients and the basic equations to build chemical evolution models. Since these fundamental papers, a great deal of work has appeared on this subject and in particular concerning the Milky Way. Many models solving numerically the basic equations of chemical evolution appeared, and in this way it was possible to relax the so-called instantaneous recycling approximation (I.R.A.), which neglects the stellar lifetimes by assuming that stars of masses $M \geq 1 M_{\odot}$ die instantaneously, while those with $M<1 M_{\odot}$ live forever. In fact, by relaxing I.R.A. we can follow the evolution of the abundance of every single chemical species. This is very important, since different chemical elements are produced on different timescales by stars of different masses. Among the first numerical models relaxing I.R.A., we recall those of Chiosi (1980) and Tosi (1982). In general, in such models, it is possible to follow the chemical enrichment by different supernova $(\mathrm{SN})$ types and by single stars ending their lives as white dwarfs (WDs). The first attempt to include the chemical enrichment by Type Ia $\mathrm{SNe}$ (assumed to be WDs in binary systems) was by Greggio and Renzini (1983); Matteucci and Tornambe (1985) and Matteucci and Greggio (1986): in particular, in the latter two papers the SNIa rate was included in a detailed chemical evolution model relaxing I.R.A., and following the evolution of several chemical species, such as $\mathrm{He}, \mathrm{C}, \mathrm{N}, \mathrm{O}, \alpha$-elements and Fe. This allowed the authors to predict the evolution of several $[\alpha / \mathrm{Fe}]^{1}$ ratios $(\alpha=\mathrm{O}, \mathrm{Si}, \mathrm{Mg})$ resulting positive and around $\sim+0.4$ dex in halo stars (with $[\mathrm{Fe} / \mathrm{H}]<-1.0 \mathrm{dex}$ ), and decreasing in disk stars until reaching the solar value $([\mathrm{Fe} / \mathrm{H}]=0 \mathrm{dex})$. At that time, there were data only for $[\mathrm{O} / \mathrm{Fe}]$ in halo stars, and so the results for $\mathrm{Si}$ and $\mathrm{Mg}$ were just predictions. Those predictions were then confirmed by François (1986), who found indeed overabundances of $\mathrm{Si}$ and $\mathrm{Mg}$ relative to $\mathrm{Fe}$ in halo stars. The interpretation of the behaviour of the $[\alpha / \mathrm{Fe}]$ ratios was that in the early phases of Galactic evolution only $\alpha$-elements are produced by short living massive stars (core-collapse $\mathrm{SNe}$, hereafter $\mathrm{CC}-\mathrm{SNe}$ ), thus reflecting the

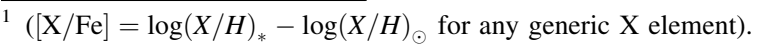


production ratios of these elements by massive stars, then when supernovae Ia (SNeIa) start to die, with a time delay relative to $\mathrm{CC}-\mathrm{SNe}$, and produce the bulk of $\mathrm{Fe}$, the $[\alpha / \mathrm{Fe}]$ ratios decrease, during the disk phase, until they reach the solar value. This interpretation is now known as time-delay model.

In the late 1980s and 1990s, other papers appeared and they were dealing with the chemical evolution of the Milky Way by means of numerical models (Tosi 1988a; Matteucci and Francois 1989, 1992; Prantzos et al. 1993; Chiappini et al. 1997; Boissier and Prantzos 1999; among others). It is worth reminding also a review paper by Matteucci (1996) and two monographs by Pagel (1997) and Matteucci (2001) on chemical evolution. Most of the models in the 1980s and 1990s were assuming that the Galactic disk formed by infall of gas, and to reproduce the G-dwarf metallicity distribution they all suggested infall timescales larger than 3-4 Gyr at the solar vicinity. Matteucci and Francois (1989) computed a model in which the disk of the Milky Way formed inside out, namely, on much shorter timescales in the inner than the outer regions, to reproduce the negative abundance gradients found along the thin disk. Such a mechanism has been found later also in complex cosmological simulations of galaxy formation (Samland and Gerhard 2003; Kobayashi and Nakasato 2011; Minchev et al. 2014; Grand et al. 2017; Domínguez-Tenreiro et al. 2017; Vincenzo and Kobayashi 2020). The inside-out disk formation was originally suggested by Larson (1976) by means of chemodynamical models: he suggested that the disk forms by gas accretion occurring faster in the inner than in outer regions.

Chiappini et al. (1997) proposed the so-called "two-infall" model, where the Galaxy formed by means of two major episodes of gas infall, the first giving rise to the stellar halo and thick disk, while the second forming the thin disk on a much longer timescale (7-8 Gyr in the solar neighbourhood); moreover, the model predicts a gap in the star formation due to an assumed gas threshold, as suggested by Kennicutt (1989). The two-infall scenario was inspired by the $[\alpha / \mathrm{Fe}]$ vs. $[\mathrm{Fe} / \mathrm{H}]$ relation observed in solar vicinity stars by Gratton et al. (1996, 2000), and subsequently confirmed by Fuhrmann (1998). In such studies, a gap appeared in the star formation process between the formation of the halo and disk stars. The long infall timescale for the assembly of the thin disk at the solar ring, was later confirmed by subsequent papers, such as Boissier and Prantzos (1999), Chiappini et al. (2001) and Alibés et al. (2001). This long timescale contrasted with the conclusions of the seminal paper of Eggen et al. (1962), where the authors inferred, from the motions and chemical composition of stars, the mechanism of the formation of the Milky Way and suggested that it happened by gas collapse on a timescale no longer than $300 \mathrm{Myr}$. However, the more precise data and in particular the G-dwarf metallicity distribution appeared in the 1980s, 1990s and early 2000s did not allow us to suggest such a short collapse timescale. On the other hand, Searle and Zinn (1978) had already challenged the short timescale of Eggen et al. (1962) for the formation of the outer stellar halo, by suggesting that it should have assembled on a timescale larger than the inner halo. Moreover, the timescale of 300 Myr is too short even for the formation of the inner halo-thick disk, which appears to be rather $\sim 1$ Gyr as suggested by Matteucci and Greggio (1986) and Chiappini et al. (1997). In recent times, it has been possible to distinguish not only among halo 
and disk stars but also among thick and thin disk stars. The existence of a structure called "thick disk" was suggested by Yoshii (1982) and Gilmore and Reid (1983), who found two distinct stellar density distributions both exponential and related to the thick and thin disk, respectively. The stars in the thick disk are characterized by high $[\alpha / \mathrm{Fe}]$ ratios, similar to halo stars, but kinematically separated either from the halo or the thin disk stars. Models including the thick disk explicitely (in Chiappini et al. 1997 model the thick disk was considered together with the halo) have appeared such as that of Pardi et al. (1995) and then Micali et al. (2013); these latter suggested a "three-infall model" with the halo forming on a time scale of $\sim 0.2$ Gyr, the thick disk on $\sim 1.25 \mathrm{Gyr}$ and the thin disk at the solar vicinity on $\sim 6$ Gyr. In this model and all of the previous ones, the star formation rate (SFR) in halo and thick disk is assumed to be more intense than in thin disk (for an extensive discussion of the models of the 2000s, see Prantzos 2008a and Matteucci 2012). Other approaches to the chemical evolution of the Milky Way halo, such as the stochastic inhomogeneous mixing and the accretion of extant stellar systems will be discussed in Sect. 5.

More recently, it has been evident that the $[\alpha / \mathrm{Fe}]$ vs. $[\mathrm{Fe} / \mathrm{H}]$ diagram shows a clear bimodal distribution between stars with high and low $[\alpha / \mathrm{Fe}]$ ratios; the former are attributed to the thick disk while the latter to the thin disk. Such a bimodality is particularly evident in APOGEE data (Anders et al. 2014; Nidever et al. 2014; Hayden et al. 2015; Queiroz et al. 2020). By analyzing the High Accuracy Radial velocity Planet Searcher (HARPS) spectra of local solar twin stars, Nissen et al. (2020) found that the age-metallicity distribution has two distinct populations with a clear age dissection. The authors suggested that these two sequences may be interpreted as evidence of two episodes of accretion of gas onto the Galactic disk with quenching of star formation in between them. To explain that, the two-infall model has been applied to the thick and thin disk. In this scenario, the thick disk forms first by accretion of primordial gas with a high star formation efficiency, whereas the thin disk forms with a time delay, due to the stop in the star formation induced by the threshold in the gas density, on a much longer timescale and from accretion of fresh primordial gas together with the gas leftover from the thick disk formation (Grisoni et al. 2017; Spitoni et al. 2019, 2020). In particular, in Grisoni et al. (2017), aiming at reproducing AMBRE (Archéologie avec Matisse Bosée sur les aRchives de l'ESO) data (de Laverny et al. 2013), the gap in the star formation between the assembly of the two disks occurs on a timescale no longer than $1 \mathrm{Gyr}$, whereas in Spitoni et al. (2019), aimed at reproducing the chemical and age data of Silva Aguirre et al. (2018), a gap of 4.3 Gyr is suggested. On the other hand, Grisoni et al. (2017) presented also a parallel scenario, where the two Galactic components formed and evolved in parallel in a completely independent way. In both the approaches presented by Grisoni et al. (2017) (two-infall and parallel), the thick disk formed on a timescale of $\sim 0.1 \mathrm{Gyr}$ and the thin disk at the solar ring of $\sim 7$ Gyr.

Cosmological models have also suggested infall of gas as a solution to explain the observed bimodality in thick and thin disk stars (e.g., Calura and Menci 2009; Buck 2020). However, it is worth noting that the existing differences in data of different surveys may indeed lead to different conclusions. What appears as a 
common feature to all chemical models is that the thick disk should have formed quickly and with intense star formation, to produce $\alpha$-enhanced stars.

Concerning the formation and evolution of the stellar halo, we remind the pioneering work of Hartwick (1976), who studied the metallicity distribution function (MDF) of globular clusters (GCs) and found that is different from the G-dwarf metallicity distribution, since it contains more metal poor objects. He devised a simple model for the Galactic halo to reproduce the MDF for GCs, where removal of gas from the halo and consequent formation of the disk was assumed. In the following years, it was suggested that the inner and outer halo might have formed on different timescales (Searle and Zinn 1978) and that part of the halo could have formed by accretion of stars from small satellites of the Galaxy, such as dwarf sheroidals (dSphs) or dwarf irregulars (DIs) or Ultra faint Dwarfs (UfDs). In particular, the accretion of satellite galaxies is predicted by the $1 \mathrm{CDM}$ paradigm, assuming that dark matter halos form hierarchically via a series of mergers with smaller halos. As a consequence, it is expected that the stellar Galactic halo might have formed from disrupted and accreted satellites (Johnston et al. 1996; Helmi and White 1999; Bullock et al. 2001; Font et al. 2005; Robertson et al. 2005; Helmi 2008, amongst others). The comparison between abundances and abundance patterns between Galactic halo stars and stars in dwarf satellites can help in understanding the formation of the halo (see Geisler et al. 2007; Matteucci 2012; Spitoni et al. 2016). In particular, all halo stars show overabundances of $\alpha$-elements relative to $\mathrm{Fe}$, while stars in $\mathrm{dSphs}$ and UfDs present a small fraction of $\alpha$-enhanced stars and many stars with low $[\alpha / \mathrm{Fe}]$ ratios at low metallicity (e.g., Tolstoy et al. 2009). Therefore, the situation is still unclear. Very recently, Helmi et al. (2018) have suggested that the inner Galactic halo is dominated by debris from an object that infalled 10 Gyr ago and it was as large as the Small Magellanic Cloud (SMC) and they called this object Gaia-Enceladus. This conclusion was deduced from the chemical and dynamical analysis of two large stellar surveys (Gaia-ESO and APOGEE). These studies hinted that this accretion event might have been responsible also for the formation of the thick disk.

The Galactic bulge is made mainly by old stars with a large range of metallicities, but with lack of very metal poor objects. Its characteristics are intermediate between a "classical bulge" and a "pseudo-bulge". There is evidence that in the bulge there are at least two stellar populations (e.g., Hill et al. 2011; Zoccali et al. 2017), one showing the characteristics of a classical bulge and the other compatible with secular evolution of the inner disk through the formation of a bar and boxy/peanut structure. Other studies (Bensby et al. 2011, 2013, 2017; Ness and Freeman 2016) suggested even multiple stellar populations in the bulge.

The first detailed chemical evolution model of the Galactic bulge was by Matteucci and Brocato (1990), who predicted that the bulk of bulge stars should have formed quickly during a burst of star formation, and should exhibit $[\alpha /$ $\mathrm{Fe}]>0$ dex for a large range of metallicities, as expected from the time-delay model, a result later confirmed by the first very detailed abundances derived by McWilliam and Rich (1994). In the following years, a great deal of chemical models appeared and they all confirmed, after comparison with the data, the fast formation of the majority of bulge stars (Ballero et al. 2007; Cescutti and Matteucci 2011; 
Grieco et al. 2012b; Matteucci et al. 2019, among others). Chemo-dynamical models based on accretion of substructures in the framework of $\Lambda \mathrm{CDM}$, have also concluded that a fast bulge formation should be preferred (Samland and Gerhard 2003; Immeli et al. 2004; Kobayashi and Nakasato 2011). In particular, Samland and Gerhard (2003) predicted that the bulge should contain two stellar populations, an old one formed during the proto-Galactic collapse and a young bar population. Other, dynamical simulations have suggested that the old bulge population originated from the thick disk stars and those belonging to the peanut structure (the bar population) from the thin disk (e.g. Di Matteo et al. 2015; Bekki and Tsujimoto 2011). Abundance data for stars in the very inner bulge have also become available (Ryde et al. 2016) and the derived abundance patterns seem to confirm a fast formation with intense SFR of this part of the bulge, when compared to theoretical models. Finally, we recall a recent and exhaustive review on cosmic chemical evolution by Maiolino and Mannucci (2019).

In this review, in Sect. 2, we will remind the basic assumptions and the main ingredients necessary to build chemical evolution models. In Sect. 3, we will present analytical solutions for chemical evolution models. In Sect. 4, we will discuss the basic equations of numerical models for the chemical evolution of the Milky Way. Section 5 will be dedicated to the description of historical model approaches. Section 6 will present the results of numerical models for the evolution of heavy elements in halo and disk. Section 7 will contain results on abundance and abundance ratio gradients along the thin disk. In Sect. 8, we will discuss stellar migration, and in Sect. 9, the evolution of light elements. Section 10 will present results for the Galactic bulge, while Sect. 11 will describe the results for the Galactic stellar halo. Section 12 will be dedicated to the chemo-dynamical models of the Milky Way in a cosmological context and comparison with pure chemical models. Finally, in Sect. 13 we will present a discussion and conclusions.

\section{Basic ingredients to build chemical models}

The basic ingredients necessary to build a chemical evolution model are:

\subsection{Initial conditions}

As initial conditions, we can assume an open or closed model, namely, if the total mass of the system is constant or variable in time. In other words, we can assume that all the gas, out of which stars will form, is present at the time $t=0$, or that it will be accreted in time. Moreover, we should assume whether the initial gas has a primordial (only light elements from the Big Bang) or metal enriched chemical composition. This latter case is known as prompt initial enrichment (PIE), and it can be created by an initial generation of massive zero metal stars (Population III stars). 


\subsection{Stellar birthrate function $($ SFR $\times$ IMF)}

The stellar birthrate function is the history of star formation in a galaxy and it can be expressed as the product of the SFR times the initial mass function (IMF). In other words, the stellar birthrate function, namely, the number of stars formed in the time interval, $(t, t+\mathrm{d} t)$ and in the mass interval $(m, m+\mathrm{d} m)$, can be written as

$$
B(m, t) \mathrm{d} m \mathrm{~d} t=\psi(t) \cdot \phi(m) \mathrm{d} m \mathrm{~d} t,
$$

where the function $\psi(t)$ represents the SFR and it is generally assumed to be only a function of time, whereas $\phi(m)$ is the IMF which is assumed to be only a function of mass. The SFR represents how many solar masses go into stars per unit time, while the IMF describes the distribution of stars at birth as a function of stellar mass. Clearly, these hypotheses are semplifications and we do not know whether the SFR is independent of mass and the IMF independent of time. Besides that, there is a sort of indetermination principle in the definition of $B(m, t)$, since in order to know the SFR we need to assume an IMF, and viceversa.

\subsubsection{Parametrization of the SFR}

The most common parametrization is the Schmidt (1959) law, where the SFR is proportional to some power $k$ of the gas volume density. Kennicutt (1998) suggested a star formation law depending on the surface gas density, as deduced by data relative to local star forming galaxies. Other important parameters such as gas temperature, viscosity and magnetic field are usually ignored.

In general, the SFR can be written as

$$
\psi(t)=v \sigma_{\mathrm{gas}}(t)^{k}
$$

where $\sigma_{\text {gas }}^{k}$ is the gas surface mass density and $v$ is the efficiency of star formation, namely, the SFR per unit mass of gas, and is expressed in units of $t^{-1}$.

In particular, the formula suggested by Kennicutt is

$$
\psi(t)=(2.5 \pm 0.7) \times 10^{-4}\left[\sigma_{\mathrm{gas}}(t)\right]^{1.4 \pm 0.15} M_{\odot} \mathrm{yr}^{-1} \mathrm{kpc}^{-2},
$$

where the efficiency parameter is derived from the fit to the SFR of local star forming galaxies.

\subsubsection{Parametrization of the IMF}

The IMF, namely, the number of stars born in the mass interval, $m-m+d m$, is generally expressed as a power law. It is possible to measure the IMF only in the solar vicinity, since one needs to count the stars as functions of their magnitudes and it is not yet possible to do this in external galaxies. Therefore, the only observational information we have is relative to the solar region of our Galaxy.

A typical expression of the IMF is 


$$
\phi(m) \mathrm{d} m=C m^{(1+x)} \mathrm{d} m,
$$

where $C$ is the so-called normalization constant obtained from the following condition:

$$
\int_{0.1}^{100} m \phi(m) \mathrm{d} m=1 .
$$

The well-known Salpeter (1955) IMF has $x=1.35$ over the whole range of stellar masses $\left(0.1-100 M_{\odot}\right)$ and is widely used in galactic evolutionary models. It should be noted that also other mass ranges, including stars up to $120 M_{\odot}$ and down to $0.05 M_{\odot}$, have been adopted in Prantzos et al. (2018) and Kobayashi et al. (2020a), respectively. However, more realistic IMFs derived for the solar vicinity have two or three slopes in the whole range of masses, such as those in Figs. 1 and 2 (Kroupa et al. 1993; Chabrier 2003; Kroupa 2001).

\subsection{Stellar nucleosynthesis}

The chemical enrichment in galaxies is due to the masses of chemical elements produced by stars of different initial mass and restored to the interstellar medium

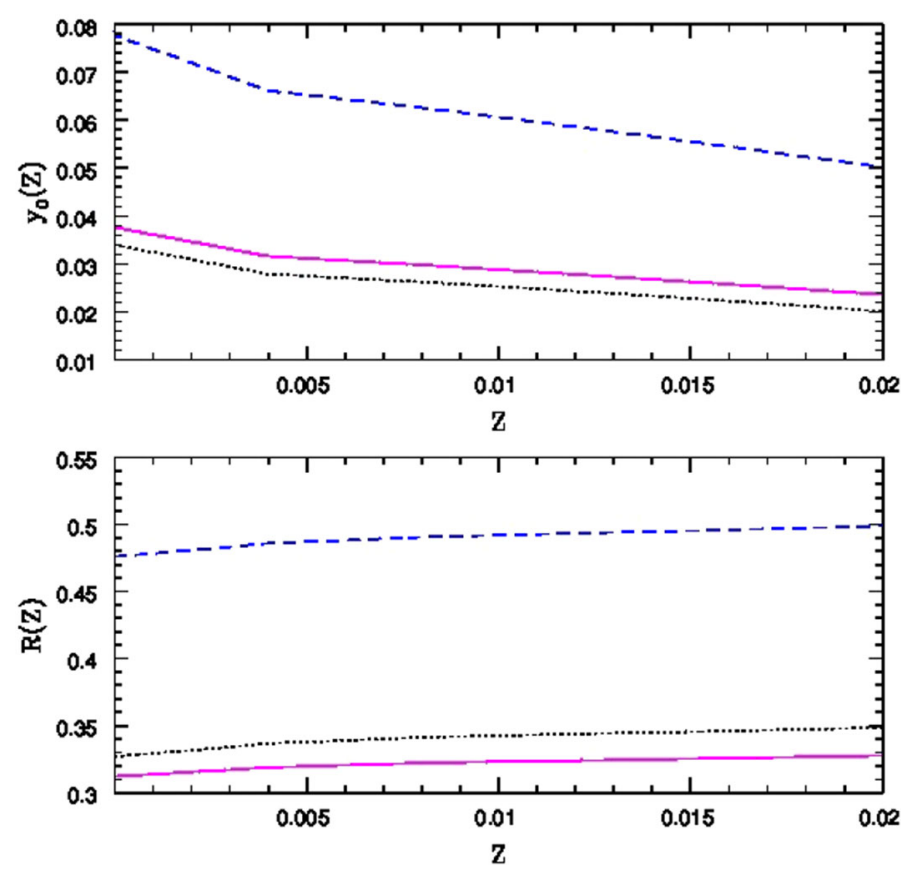

Fig. 1 Upper panel: the yield of oxygen per stellar generation computed for different metallicities and IMFs. The blue dotted line refers to Chabrier (2003) IMF, the magenta line is the Salpeter (1955) IMF and the black line is the Kroupa et al. (1993) IMF. Lower panel: returned fraction R as a function of IMF and metallicity. Image reproduced with permission from Vincenzo et al. (2016), copyright by the authors 


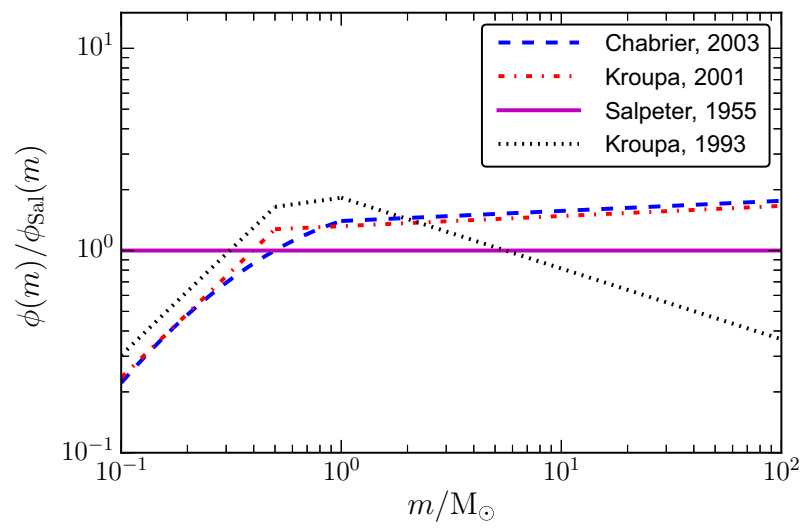

Fig. 2 Comparison between the different IMFs adopted in Fig. 1, normalized to the Salpeter IMF. Image reproduced with permission from Vincenzo et al. (2016), copyright by the authors

(ISM) when they die. These masses represent both the newly formed elements and the elements already present in the star at its formation and restored into the ISM without being reprocessed.

In particular, we can define the mass of a newly formed element in a star of mass $m$ as

$$
M_{i m}=\int_{0}^{\tau_{m}} \dot{M}_{\text {lost }} \cdot\left[X(i)-X_{o}(i)\right] \mathrm{d} t,
$$

where $\tau_{m}$ is the lifetime of a star of mass $m, X_{o}(i)$ is the original abundance of the element $i, X(i)$ is the final one and $\dot{M}_{\text {lost }}$ is the mass loss rate of the star.

With this quantity we can define what is called stellar yield, namely:

$$
p_{\text {im }}=M_{\text {im }} / m \text {. }
$$

To obtain the total stellar mass ejected, we should add to Eq. (6) the mass ejected without being processed, namely:

$$
M_{\text {imo }}=X_{o}(i) \cdot M_{\mathrm{lost}},
$$

where $M_{\text {imo }}$ is the stellar mass in the form of the element $i$, already present in the star at birth. With $M_{\text {lost }}$ we intend the total mass ejected by the star into the ISM during its lifetime.

Each stellar mass can produce and eject different chemical elements and the yields are a function of the stellar mass but also of the original stellar metal content that we will indicate with $Z$. These yields are computed by means of detailed nucleosynthesis calculations taking into account all the main nuclear reactions in stars.

Here, we summarize briefly the element production in stars: (i) stars with $M<0.8 M_{\odot}$ do not contribute to galactic chemical enrichment, since they have lifetimes longer than the Hubble time. (ii) Low and intermediate mass stars $(0.8-$ $8 M_{\odot}$ ) produce $\mathrm{He}, \mathrm{N}, \mathrm{C}$ and heavy s-process elements (e.g., Ba, Y, Sr). They die as 
C-O WDs when single, and can die as Type Ia SNe when in binaries. Type Ia SNe are, in fact, believed to originate in WDs in binary systems. (iii) Massive stars $\left(M>8-10 M_{\odot}\right)$ produce mainly alpha-elements $(\mathrm{O}, \mathrm{Ne}, \mathrm{Mg}, \mathrm{S}, \mathrm{Si}, \mathrm{Ca})$, some $\mathrm{Fe}$, light s-process elements and perhaps r-process elements and explode as CC-SNe. However, r-process elements originating in neutron binary mergers seems to represent one of the most promising channel for r-process element production (Korobkin et al. 2012; Eichler et al. 2015), especially after the GW170817 gravitational-wave event connected to merging neutron stars and the detection of heavy elements (e.g., Evans et al. 2017; Pian et al. 2017; Tanvir et al. 2017; Troja et al. 2017). On the other hand, Côté et al. (2019) and Kobayashi et al. (2020a) concluded that magneto-rotational supernovae could be the main site of the $r$ process in the Galaxy. (iv) Novae are binary systems made of a WD plus a Main Sequence or Red Giant low mass star. These systems suffer outbursts which do not destroy the WD, but create new elements through explosive H-burning. These new elements are: $\mathrm{CNO}$ isotopes $\left({ }^{13} \mathrm{C},{ }^{15} \mathrm{~N},{ }^{17} \mathrm{O}\right)$, perhaps ${ }^{7} \mathrm{Li}$, plus some radioactive elements (e.g., ${ }^{22} \mathrm{Na},{ }^{26} \mathrm{Al}$ ) (see José and Hernanz 2007).

To summarize: CC-SNe do produce the bulk of $\alpha$-elements and only part of Fe on timescales negligible relative to a Hubble time, whereas Type Ia $\mathrm{SNe}$ do produce the bulk of Fe plus traces of elements from $\mathrm{C}$ to $\mathrm{Si}$ on a large range of timescales, going from $35 \mathrm{Myr}$ to a Hubble time. Low and intermediate mass stars dying in the same range of times as Type Ia $\mathrm{SNe}$, do produce the bulk of heavy s-process elements, ${ }^{14} \mathrm{~N}$ and part of $\mathrm{He}$ and ${ }^{12} \mathrm{C}$. Novae can contribute to the enrichment of $\mathrm{CNO}$ isotopes, ${ }^{7} \mathrm{Li}$ and radioactive elements on long timescales, whereas merging neutron stars (MNS) can contribute substantially to r-process elements (e.g., Eu) either on short or moderately long merging timescales (see later).

\subsubsection{The yield per stellar generation}

To understand the chemical enrichment by a simple stellar population, namely, stars born at the same time and with the same chemical composition, we define the yield per stellar generation of a single chemical element, as in Tinsley (1980)

$$
y_{i}=\frac{\int_{1}^{\infty} m p_{i m} \phi(m) \mathrm{d} m}{(1-R)},
$$

where $p_{i m}$ is the stellar yield of the newly produced and ejected element $i$ by a star of mass $m$, as defined before, and $R$ is the returned fraction (see Eq. 10). Therefore, the yield $y_{i}$ is the mass fraction of the element $i$ newly produced by a generation of stars, relative to the fraction of mass locked up in remnants (white dwarfs, neutron stars and black holes) and brown dwarfs $\left(M<0.1 M_{\odot}\right)$.

We define returned fraction the fraction of mass ejected into the ISM by an entire stellar generation, namely: 


$$
R=\frac{\int_{1}^{\infty}\left(m-M_{\mathrm{rem}}\right) \phi(m) \mathrm{d} m}{\int_{0.1}^{\infty} m \phi(m) \mathrm{d} m}
$$

where $M_{\text {rem }}$ is the remnant mass, which can be either a WD or a neutron star or a black hole.

The term fraction originates from the fact that $R$ is divided by the normalization integral of the IMF, which is equal to unity (Eq. 5). The upper mass limits here is indicated by $\infty$ but normally is assumed to be $100 M_{\odot}$.

To define $y_{i}$ and $R$, we have made a very specific assumption: the instantaneous recycling approximation (I.R.A.), stating that all stars more massive than $1 M_{\odot}$ die instantaneously, while all stars less massive than $1 M_{\odot}$ live forever. This assumption allows us to solve analytically the chemical evolution equations, but it is a very poor approximation for chemical elements produced partly or entirely on long timescales, such as $\mathrm{C}, \mathrm{N}$ and Fe. On the other hand, for oxygen, which is almost entirely produced by short lived CC-SNe, I.R.A. can be an acceptable approximation. In Fig. 1, we report the yield per stellar generation of oxygen, $y_{O}$, as well as the returned fraction, $R$, computed for different initial stellar metallicities and three different IMFs (Chabrier 2003; Salpeter 1955; Kroupa et al. 1993). As one can see, the variation of $y_{O}$ and $R$ with metallicity $Z$ is negligible, whereas the dependence on the assumed IMF is strong. In particular, the Chabrier (2003) IMF predicts the largest differences in these two quantities, and the reason can be found in the larger number of massive stars in this IMF, relative to the other ones (see Fig. 2). In Fig. 1, the Kroupa (2001) universal IMF, suggesting that the IMF in stellar clusters is an universal one, does not appear and the reason is that is very similar to that of Chabrier (2003), as one can see in Fig. 2. Finally, the IMF of Kroupa et al. (1993), obtained for the solar vicinity, contains less massive stars than all the other IMFs adopted by Vincenzo et al. (2016). The adopted stellar yields to compute $y_{O}$ and $R$ are those of Romano et al. (2010) their model 15.

\subsection{Gas flows: infall, outflow, radial flows}

To build a realistic galaxy one has to assume the presence of gas flows both in and out. The gas inflows are considered either as gas accretion or radial gas flows and they are influencing the chemical evolution of galaxies: in the case of accretion, usually assumed to occur at a constant rate or exponentially decreasing in time, the main effect is to dilute the metal content, except if the metallicity of the infalling gas is equal or larger than that of the pre-existing gas, but this is a rather unlikely situation.

The most common parametrization of the gas infall is

$$
A(t)=\Lambda e^{-t / \tau},
$$

where $A(t)$ is the gas accretion rate, namely, how many solar masses are accreted per unit time, $\Lambda$ (adimensional) and $\tau$ are two free parameters. In particular, $\tau$ is the timescale for gas accretion, namely, the time necessary to accumulate half of the mass of the system. In some analytical chemical models (e.g., Matteucci and Chiosi 
1983), it has been assumed that $A(t) \propto \psi(t)$, but this is a questionable assumption, since is indeed the SFR which is affected by $A(t)$ but not viceversa.

In the case of outflows or galactic winds (when the mass is lost from the galactic potential well), the effect is also that of decreasing the metal concentration by simply decreasing the gas which is available for star formation.

Galactic outflows are generally assumed to occur at a rate proportional to the SFR, such as

$$
W(t)=-\lambda \psi(t)
$$

where $W(t)$ is the wind rate, namely, how many solar masses are lost per unit time from the galaxy, $\lambda$ is a free adimensional parameter and $\psi(t)$ is the SFR.

In the case of radial gas flows, the most common assumption is that they are directed inward, as a dynamical consequence of gas infalling onto the disk. In fact, the infalling gas has a lower angular momentum than the circular motion in the disk, and mixing with the gas in the disk produces a net radial inflow. Such an inflow can favour the formation of abundance gradients as long as its speed is $<2 \mathrm{~km} / \mathrm{s}$ (Tinsley 1980). Many models for chemical evolution of the Milky Way have assumed inward radial gas flows in the disk (Mayor and Vigroux 1981; Lacey and Fall 1985; Goetz and Koeppen 1992; Portinari and Chiosi 2000; Schönrich and Binney 2009; Spitoni and Matteucci 2011; Grisoni et al. 2018; Vincenzo and Kobayashi 2020).

\section{Analytical models of chemical evolution}

First of all, we discuss the so-called Simple Model for the chemical evolution of the solar neighbourhood. We note that the solar neighbourhood is defined as a region centered in the Sun and extending roughly $1 \mathrm{kpc}$ in all directions.

\subsection{Basic assumptions and solution of the Simple Model}

In this paragraph we follow the definition of the Simple Model as given in Tinsley (1980); in particular, the Simple Model is based on the following assumptions:

1. the system is one-zone and closed, namely, there are no inflows or outflows,

2. the initial gas is primordial,

3. $\varphi(m)$ is constant in time,

4. the gas is well mixed at any time.

The well known solution of the Simple Model is

$$
Z=y_{Z} \ln \left(\frac{1}{\mu}\right) \text {, }
$$

where $\mu=\frac{M_{\mathrm{gas}}}{M_{\mathrm{tot}}}$ is the gas mass fraction ( $M_{\mathrm{tot}}$ is the mass of stars plus gas), and $y_{Z}$ is the yield per stellar generation of the metals. This solution is obtained after 
assuming I.R.A. and integrating the equation describing the evolution of $Z$ between $M_{\text {gas }}(0)=M_{\text {tot }}$ and $Z(0)=0$ and $Z(t)$.

The yield which appears in Eq. (13) is known as effective yield, and is simply defined as the yield $y_{Z_{\text {eff }}}$ that would be deduced if the system were assumed to be described by the Simple Model. Therefore

$$
y_{Z_{\mathrm{eff}}}=\frac{Z}{\ln (1 / \mu)} .
$$

The effective yield represents the highest degree of chemical enrichment for a given IMF. In fact, systems, where the hypothesis 1) is relaxed (i.e., infall and/or outflow) have true yields lower than the effective yield (see later). The Simple Model for describing the evolution of the solar vicinity was discarded, since it predicts too many long living stars (G-dwarfs) at low metallicities, the well known "G-dwarf problem", which was solved by assuming gas infall for the formation of the solar vicinity. Moreover, this model and also all the analytical chemical models, cannot follow, because of the I.R.A., the evolution of elements restored into the ISM on long timescales, such as Fe, which is formed mainly in Type Ia SNe and is the main tracer of stellar metallicity. On the other hand, for elements formed on short timescales by massive stars, such as $\mathrm{O}$, the analytical solutions assuming I.R.A. can be acceptable.

The solution of Eq. (13) is valid for a primary element, namely, an element formed directly from $\mathrm{H}$ and $\mathrm{He}$, as opposed to a secondary element which is formed from metals already present in the star at birth. For the abundance of a secondary element $X_{S}$, such as ${ }^{14} \mathrm{~N}$, which is produced during the CNO cycle, although it can have also a primary origin if the $\mathrm{C}$ and $\mathrm{O}$ out of which is formed have been synthesized in the star (cases of dredge-up in AGB stars and rotation in massive stars), the solution of the Simple Model is (Tinsley 1980)

$$
X_{S}=\frac{1}{2}\left(\frac{y_{S}}{y_{Z} Z_{\odot}}\right) Z^{2},
$$

where $y_{S}$ is the yield per stellar generation for the generic secondary element $S$. As one can see from Eq. (15), the ratio between the abundance of a secondary element and the abundance of its primary progenitor evolves proportionally to the abundance of the progenitor (e.g., $X_{S} / Z \propto Z$ ).

\subsection{Analytical solution for gas outflows}

A more realistic situation would involve gas flows in the studied system, in particular outflow and infall. The situation in which there is only gas outflow can be described by the following solution (Matteucci and Chiosi 1983)

$$
Z=\frac{y_{Z}}{(1+\lambda)} \ln \left[(1+\lambda) \mu^{-1}-\lambda\right]
$$

After assuming a wind rate of the form 


$$
W(t)=-\lambda(1-R) \psi(t),
$$

where $\lambda \neq 0$ is the adimensional wind parameter, and integrating between 0 and $\mathrm{t}$ and between $M_{\text {tot }}=M_{\text {gas }}(0)$ and $M_{\text {gas }}(t)$.

It is clear that for $\lambda=0$ Eq. (16) becomes the solution of the Simple Model (Eq. 13). The meaning of Eq. (16) is immediately clear, the true yield is lower than the effective yield in presence of only outflows.

\subsection{Analytical solution for gas infall}

In the case of only gas infall, the solution for a primordial chemical composition $\left(Z_{\text {inf }}=0\right)$ of the infalling gas is (Matteucci and Chiosi 1983)

$$
Z=\frac{y_{Z}}{\Lambda}\left[1-\left(\Lambda-(\Lambda-1) \mu^{-1}\right)^{-\Lambda /(1-\Lambda)}\right]
$$

where the accretion rate has been chosen to be:

$$
A(t)=\Lambda(1-R) \psi(t)
$$

with $\Lambda$ a positive constant different from zero and from 1 . In addition, in this case, the true yield in Eq. (18) is lower than the Simple Model effective yield, and by imposing $\Lambda=0$ the Eq. (18) becomes the solution of the Simple Model.

If $\Lambda=1$ the solution is

$$
Z=y_{Z}\left[1-e^{-\left(\mu^{-1}-1\right)}\right],
$$

which is the well-known solution for the extreme infall case (Larson 1972; Tinsley 1980), where the amount of gas remains constant in time.

\subsection{Analytical solution for gas infall plus outflow}

If $A(t)=\Lambda(1-R) \psi(t)$ and $W(t)=-\lambda(1-R) \psi(t)$ are both active, the analytical solution is (Recchi et al. 2008)

$$
Z=\frac{\left(\Lambda Z_{\text {inf }}+y_{Z}\right)}{\Lambda}\left[1-(\Lambda-\lambda)-(\Lambda-\lambda-1) \mu^{-1}\right]^{\frac{\Lambda}{\Lambda-\lambda-1}},
$$

for $\Lambda \neq 0 \neq 1$ and $\lambda \neq 0$. This general solution allows us to consider also an enriched infall (i.e., $Z_{\text {inf }} \neq 0$ ). A similar situation has been studied by Lilly et al. (2013) and called "bathtube model".

\subsection{Analytical solution for biased galactic outflow plus infall}

Both theory (e.g., Vader 1986; Recchi et al. 2001, 2008) and observations (e.g., Martin et al. 2002) have suggested that galactic outflow can be metal-enhanced, in the sense that metals produced by supernovae are lost more easily from a galaxy than the total gas made mainly by $\mathrm{H}$ and He. Recchi et al. (2008) found an 
analytical solution for galactic winds carrying out mostly metals. In such a case, the wind rate is defined as

$$
W(t) Z^{o}=-\alpha_{e f} Z \lambda(1-R) \psi(t),
$$

where $Z^{o}$ is the metallicity of the outflowing gas which, in this case, can be different from the metallicity $Z$ present in the galactic gas and can be defined as:

$$
Z^{o}=\alpha_{\mathrm{ef}} Z
$$

with $\alpha_{\mathrm{ef}}>1$ being the ejection efficiency.

The equation for metals in this case is

$$
\frac{\mathrm{d}\left(Z M_{\mathrm{gas}}\right)}{\mathrm{d} t}=(1-R) \psi(t)\left[\Lambda Z_{\mathrm{inf}}+y_{Z}-\left(\lambda \alpha_{\mathrm{ef}}+1\right) Z\right],
$$

where $Z_{\text {inf }}, \Lambda$ and $\lambda$ are the same parameters as defined above. The solution of this equation is

$$
Z=\frac{\Lambda Z_{\text {inf }}+y_{Z}}{\Lambda+\left(\alpha_{\mathrm{ef}}-1\right) \lambda}\left\{1-\left[(\Lambda-\lambda)-(\Lambda-\lambda-1) \mu^{-1}\right]^{\frac{\Lambda+\left(\alpha_{e f}-1\right) \lambda}{1-\lambda-1}}\right\} .
$$

One can immediately see that if we assume $\alpha_{\mathrm{ef}}=1$, we obtain again Eq. (21).

\subsection{More complex analytical solutions}

Interesting and more refined analytical solutions are those proposed by Spitoni et al. (2017) which follow the evolution of the gas mass, total mass and metallicity of a given galaxy. In particular, these solutions are obtained for an exponential infall gas law, such as in Eq. (11) $\left(A(t)=\Lambda e^{-t / \tau}\right)$, with $\tau$ being the timescale of the infall and $\Lambda$ a normalization constant obtained from the following condition:

$$
\int_{0}^{t_{G}} \Lambda e^{-t / \tau} \mathrm{d} t=M_{\text {infall }}
$$

where $t_{G}$ is the galactic lifetime and $M_{\text {infall }}$ is the mass that is accumulated by gas infall over the time $t_{G}$.

A galactic outflow law is also taken into account with the expression:

$$
W(t)=-\lambda \psi(t),
$$

which is similar to that of Eq. (17) except for the factor $(1-R)$.

In the Spitoni et al. (2017) model formulation, there is also an explicit consideration of the SFR law, in particular:

$$
\psi(t)=v M_{\mathrm{gas}}(t),
$$

where $v$ is the efficiency of star formation, as previously defined in Eq. (2) and $M_{\text {gas }}$ is the gas mass at the time $t$.

The basic equations of this model are 


$$
\begin{gathered}
\frac{\mathrm{d} M_{\mathrm{tot}}}{\mathrm{d} t}=\Lambda e^{-t / \tau}-\lambda \psi(t) \\
\frac{\mathrm{d} M_{\mathrm{gas}}}{\mathrm{d} t}=-(1-R) \psi(t)+\Lambda e^{-t / \tau}-\lambda \psi(t) \\
\frac{\mathrm{d} M_{Z}}{\mathrm{~d} t}=\left[-Z(t)+y_{Z}\right](1-R) \psi(t)-\lambda Z(t) \psi(t)+Z_{\text {inf }} \Lambda e^{-t / \tau} .
\end{gathered}
$$

The quantity $Z_{\text {inf }}$ represents the metallicity of the infalling gas.

The equation for metals can be written as

$$
\dot{Z}(t)=y_{Z}(1-R) v+\frac{\Lambda\left[Z_{\mathrm{inf}}-Z(t)\right] e^{-t / \tau}}{M_{\mathrm{gas}}(t)} .
$$

The integration of the above equations is performed with the following initial conditions: at $t=0 M_{\mathrm{tot}}(0)=M_{\mathrm{gas}}(0)$. The initial metallicity $Z(0)=0$ and the chemical composition of the infalling gas is primordial $\left(Z_{\text {inf }}=0\right)$. The solution for the gas mass is

$$
M_{\mathrm{gas}}(t)=e^{-\alpha t}\left[\frac{\Lambda /\left(e^{-t / \tau+\alpha t}-1\right) \tau}{\alpha \tau-1}+M_{\mathrm{gas}}(0)\right],
$$

and for the global gas metallicity $Z$ :

$$
Z(t)=\frac{y_{Z} v(1-R)}{\alpha \tau-1} \cdot \frac{M_{\mathrm{gas}}(0) t(\alpha \tau-1)^{2}+\Lambda \tau\left[t-\tau(1+\alpha t)+\tau e^{-\alpha t-t / \tau}\right]}{\Lambda \tau\left(e^{\alpha t-t / \tau}-1\right)+M_{\mathrm{gas}}(0)(\alpha \tau-1)}
$$

In the two equations above it has been assumed $\alpha=(1+\lambda-R) v$.

It is interesting to compute also the average metallicity of stars $\left(\left\langle Z_{*}(t)\right\rangle\right)$ which can be expressed as

$$
\left\langle Z_{*}(t)\right\rangle=\int_{0}^{t} \mathrm{~d} t^{\prime} Z\left(t^{\prime}\right) \psi\left(t^{\prime}\right) / \int_{0}^{t} \mathrm{~d} t^{\prime} \psi\left(t^{\prime}\right) .
$$

The above analytical solutions (Eqs. 33 and 34) can be very useful to study the Mass-Metallicity relation in either star forming or passive galaxies with the necessary condition of considering the global metallicity $Z$, which is dominated by the abundance of oxygen for which the I.R.A. is a good approximation. Clearly, these solutions cannot be applied to the study of the evolution of elements produced on long timescales, such as iron.

In Fig. 3, we show the effects of galactic gas outflows with wind parameter $\lambda=0.5$ and gas infall as in Eq. (11) with infall timescale $\tau=2$ Gyr. It is evident the effect of galactic winds taking out of the galaxy a large fraction of gas and, therefore, metals. 

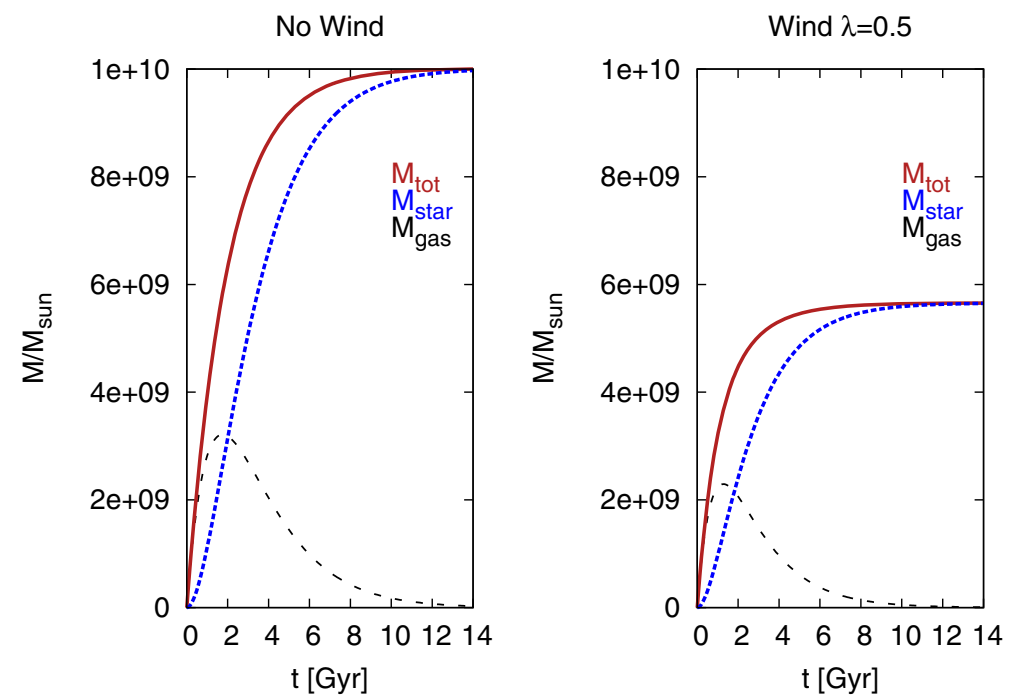

Fig. 3 Effects of galactic winds on the predicted time evolution of the galaxy gas mass $M_{\text {gas }}$ (gray dashed lines), stellar mass $M_{\star}$ (blue dotted lines), and total mass $M_{\text {tot }}=M_{\text {gas }}+M_{\star}$ (red solid lines). The model assumes an infall timescale $\tau=2 \mathrm{Gyr}$ and infall mass $M_{\text {infall }}=10^{10} M_{\odot}$, and a star formation efficiency $v=1 \mathrm{Gyr}^{-1}$. Left panel: model without galactic winds $(\lambda=0)$; Right panel: model with wind parameter $\lambda=0.5$. Image reproduced with permission from Spitoni et al. (2017), copyright by ESO

\section{Numerical chemical evolution models of the Milky Way}

We have seen in the previous section, that if we assume the I.R.A. the equations for gas and metals can be solved analytically. However, the I.R.A. is a good approximation only for the chemical elements which are produced on timescales negligible relative to the age of the Universe, but is a very bad approximation for elements produced on long timescales. The former are basically the $\alpha$-elements which are mainly produced in $\mathrm{CC}-\mathrm{SNe}$ (i.e., $\mathrm{O}$ and $\mathrm{Mg}$ ), although some $\alpha$-elements (e.g., $\mathrm{Si}$ and $\mathrm{Ca}$ ) are also produced in a non-negligible quantity by long living stars (Type Ia SNe). The latter are elements, such as $\mathrm{Fe}$ and $\mathrm{N}$, which are mainly produced by low and intermediate mass stars either by single stars (e.g., N) or binary systems (e.g., Fe from SNe Ia).

If we assume any of the analytical solutions derived before and we apply them to two single chemical elements, instead of $Z$, then we can state that the ratio of the abundances of such elements is a constant and equal to the ratio of the corresponding yields per stellar generation (see Fig. 1). Therefore, if we take for example $\mathrm{O}$ and $\mathrm{Fe}$, then we conclude that the ratio $X_{\mathrm{O}} / X_{\mathrm{Fe}}$ is constant during the entire Galactic lifetime. We know from studying the abundance ratios in the main Galactic components that this is not true. In fact, the $[\mathrm{O} / \mathrm{Fe}]$ ratio in halo stars is larger than solar and it decreases towards the solar value $([\mathrm{O} / \mathrm{Fe}]=0 \mathrm{dex})$ in disk stars (see later). To study in detail and in the correct way the evolution of the abundances of single elements we must relax I.R.A. and solve the chemical evolution equations numerically. 


\subsection{Basic equations of chemical evolution}

A suitable compact form for the complete chemical evolution equations is the following, where we write one equation for each chemical element, such as

$$
\dot{\sigma}_{i}(t)=-\psi(t) X_{i}(t)+\dot{R}_{i}(t)+\dot{\sigma}_{i, \text { inf }}(t)-\dot{\sigma}_{i, w}(t),
$$

where $\sigma_{i}(t)=\sigma_{\text {gas }}(t) X_{i}(t)$ is the surface mass density of the element $i$ in the ISM at the time $t$. The quantity $X_{i}(t)=\sigma_{i}(t) / \sigma_{\text {gas }}(t)$ is the abundance by mass of the element $i$ and $\sigma_{\text {gas }}(t)$ is the surface mass density of the ISM. The quantity $\psi(t)$ is the SFR, the quantity $\dot{R}_{i}(t)$ is the rate of variation of the returned mass in form of new and old chemical elements. The quantities $\dot{\sigma}_{i, i n f}(t)$ and $\dot{\sigma}_{i, w}(t)$ are the infall and outflow rate, respectively, and are expressed in terms of $M_{\odot} \mathrm{pc}^{-2} \mathrm{Gyr}^{-1}$.

The term $\dot{R}_{i}(t)$ can be written as

$$
\dot{R}_{i}(t)=\int_{m(t)}^{m_{\max }} \psi\left(t-\tau_{m}\right) Q m_{i}(t)\left(t-\tau_{m}\right) \phi(m) \mathrm{d} m,
$$

where $m(t)$ is the minimum mass dying at the time $t, \phi(m)$ is the IMF, $\psi\left(t-\tau_{m}\right)$ is the SFR at the time at which the star of mass $m$ was born $\left(\tau_{m}\right)$. The quantity $Q m_{i}(t)$ contains all the stellar nucleosynthesis (old and newly produced element $i$ by a star of mass $m$ ). The dependence of $Q m_{i}$ upon $\left(t-\tau_{m}\right)$ is relative to the abundance of the element $i$ already present at the star birth (the fraction of $i$ restored without being processed). This integral contains the products of all stars, such as single stars dying as WDs, SNeIa and CC-SNe.

Concerning the SFR, a common parametrization is the Schmidt-Kennicutt law expressed in Eq. (2).

The gas infall rate is generally described by an exponential law, as expressed by Eq. (11). Such a law should be written for each chemical element in the following way:

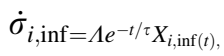

with $\sigma_{i \text {,inf }}$ being the surface gas density of the element $i$ present in the infalling gas and $X_{i \text { inf }}$ its abundance. Normally, the chemical composition of the infalling gas is assumed to be primordial (no metals). In reality, the infall metallicity is likely not primordial, but models have shown that it must be quite low (e.g., Tosi 1988b) and consistent with the metallicity observed in the Magellanic Stream, for example.

The parameter $\Lambda$ is derived by imposing the condition of Eq. (26) which gives

$$
\Lambda=\frac{\sigma_{\mathrm{inf}}\left(t_{G}\right)}{\tau\left(1-e^{-t_{G} / \tau}\right)},
$$

where $t_{G} \sim 13.7 \mathrm{Gyr}$, and $\sigma\left(t_{G}\right)_{\text {inf }}$ is the present time total surface mass density accumulated by infall (infall mass if the chemical equation is written in terms of masses and not surface densities) of the studied system. If we study the solar vicinity the total present time surface mass density is $\sim 48 \pm 8 M_{\odot} \mathrm{pc}^{-2}$ (Kuijken 
and Gilmore 1989). A more recent estimate from McKee et al. (2015) suggests $\sim 41 \pm 3.4 M_{\odot} \mathrm{pc}^{-2}$.

Finally, the rate of outflow has generally the form:

$$
\dot{\sigma}_{i, w}(t)=-w_{i} \psi(t) X_{i}(t),
$$

where $w_{i}$ is the so-called mass loading factor or wind efficiency parameter relative to the specific element $i$. It can be different for different elements (i.e., differential galactic winds).

In summary, Eq. (36) can be solved only numerically and the most complex computation is due to the term $\dot{R}_{i}(t)$. This quantity appears as an integral in Eq. (36) involving the SFR and the IMF, and it grows with the integration time, since more and more stars die at any successive timestep. It contains the detailed nucleosynthesis occurring in each star contributing to the chemical enrichment.

\subsection{The supernova rates}

The galactic chemical enrichment (the term $\dot{R}_{i}(t)$ in Eq. (36) occurs mainly by means of supernovae of all Types (Ia, Ib, Ic and II), C-O WDs, novae and MNS. Massive stars end their lives as SNe II, Ib, Ic and SNe Ib,c are originating from the upper mass range: some of these $\mathrm{SNe}$ can have a very high explosion energy ( $\sim 10^{52} \mathrm{erg}$ ), while normally is $\sim 10^{51} \mathrm{erg}$, and are called hypernovae. Therefore, it is necessary to compute in detail the SN, nova and MNS rates to estimate their chemical pollution. In the following we summarize how to compute all these rates.

\subsubsection{Rate of CC-SNe}

The rate of CC-SNe (Type II, Ib, Ic) can be computed by assuming either that they originate from single massive stars or massive binaries. In particular, the SNeII are exploding single massive stars in the mass range $\left(8 \leq M / M_{\odot} \leq M_{\mathrm{WR}}\right)$, although the upper limit, namely, the limiting mass for the formation of a Wolf-Rayet (WR) star, is very uncertain depending upon mass loss, rotation and other physical assumptions of the stellar models.

The $\mathrm{SNe} \mathrm{Ib}$ and Ic are either the explosions of single stars with $M>M_{\mathrm{WR}}$ or stars in binary systems in the mass range $12-20 M_{\odot}$ (Baron 1992) or 14.8-45 $M_{\odot}$ (Yoon et al. 2010). Finally, a fraction od CC-SNe are called "hypernovae". These SNe originate from masses $>M_{\mathrm{WR}}$ but with explosion energy $\left(\sim 10^{52} \mathrm{erg}\right)$ a factor of 10 higher than the other SNe.

The rate of Type II SNe can be written as

$$
R_{\mathrm{SNII}}(t)=\int_{8}^{M_{\mathrm{WR}}} \psi\left(t-\tau_{m}\right) \phi(m) \mathrm{d} m,
$$

while the rate for Type $\mathrm{Ib}$, Ic is given by the previous equation, where the integration goes from $M_{\mathrm{WR}}$ to $M_{\mathrm{max}}$, which can be any mass from 70 to $120 M_{\odot}$, plus the rate of explosion of massive binaries in a given range 


$$
R_{\mathrm{SNIb}, \mathrm{c}}(t)=(1-\gamma) \int_{M_{\mathrm{WR}}}^{M_{\mathrm{max}}} \psi\left(t-\tau_{m}\right) \phi(m) \mathrm{d} m+\gamma \int_{14.8}^{45} \psi\left(t-\tau_{m}\right) \phi(m) \mathrm{d} m,
$$

where $\gamma$ is a free parameter indicating how many massive binary systems are present in the range 14.8-45 $M_{\odot}$, and is normalized to obtain the present time rate of Type Ib,c SNe in galaxies $(\gamma=0.15-0.30$, Grieco et al. 2012a).

\subsubsection{Supernovae la}

The rate of SNe Ia is very important, since these SNe are believed to be the major producers of $\mathrm{Fe}$ in the Universe, which is the main tracer of stellar metallicity. Two basic scenarios for Type Ia SNe have been proposed in the last years: (i) the single degenerate (SD), where a WD in a binary system explodes after accretion of matter from a companion, which fills its Roche lobe when becoming a red giant (but it could be also a slightly evolved main sequence star), and reaches and overcomes the Chandrasekhar mass (Whelan and Iben 1973), and (ii) the double degenerate (DD), where two WDs merge after losing angular momentum because of gravitational wave emission, and explode after reaching the Chandrasekhar mass (Iben and Tutukov 1984; Han and Podsiadlowski 2004). The Chandrasekhar mass in absence of $\mathrm{H}$ is $M_{\mathrm{Ch}} \sim 1.44 M_{\odot}$ and represents the limiting mass for the stability of a $\mathrm{C}-\mathrm{O}$ WD. In the last years, many variations to these two basic scenarios have been suggested (see Hillebrandt et al. 2013; Ruiter 2020 for reviews and Kobayashi et al. 2020b), including sub-Chandrasekhar masses for the exploding WD. The alternative scenarios have been proposed to explain the so-called peculiar SNeIa. Concerning the SD scenario, a point of concern has always been the requested precise rate of mass accretion from the donor. To overcome this problem, Hachisu et al. (1999) suggested a scenario, where a wind from the WD stabilizes the accretion from the donor and provides a wider channel to the occurrence of SNeIa.

Greggio (2005) proposed a general formulation for the rate of Type Ia SNe, which can include any possible progenitor model for such supernovae, if expressed in analytical form. In this way, the SNIa rate is given by the product of the SFR and the function describing the distribution of the explosion times (delay time distribution, hereafter DTD).

In particular, we can write

$$
R_{\mathrm{SNIa}}(t)=\kappa_{\alpha} \int_{\tau_{i}}^{\min \left(t, \tau_{x}\right)} A(t-\tau) \psi(t-\tau) \operatorname{DTD}(\tau) \mathrm{d} \tau,
$$

where $\psi(t-\tau)$ is the SFR, $\tau$ is the total delay time, namely, the nuclear stellar lifetime of the secondary star plus a possible delay due for example to the gravitational time delay in the DD model; this time is defined in the range $\left(\tau_{i}, \tau_{x}\right)$, so that

$$
\int_{\tau_{i}}^{\tau_{x}} \operatorname{DTD}(\tau) \mathrm{d} \tau=1
$$

The function $\operatorname{DTD}(t)$ can be any function able to describe the sequence of SNIa explosions, the time $\tau_{i}$ is the minimum time requested for the explosion of SNeIa. In 
the SD scenario, $\tau_{i}$ is the lifetime of a $\sim 8 M_{\odot}$ star, which is considered the maximum mass for the formation of a C-O WD, although the precise value of this mass depends on the stellar evolution model prescriptions. In the DD scenario, $\tau$ is the lifetime of a $\sim 8 M_{\odot}$ star plus the minimum gravitational time delay ( $\sim 1$ Myr, Greggio 2005). The time $\tau_{x}$ is the maximum time for the explosion of a SNIa and in the SD model is the Hubble time corresponding to the lifetime of a $\sim 0.8 M_{\odot}$, whereas in the DD model this value can be several Hubble times, depending on the initial separation of the two WDs which determines the timescale for merging.

The parameter $A(t-\tau)$ is the fraction of binary systems, in the IMF, possessing the right characteristics to produce a SN Ia. It is normally considered as a constant but in principle it could vary with time. In general, $A$ is derived by reproducing the present time observed Type Ia SN rate of the object under study.

The quantity $\kappa_{\alpha}$ contains the IMF, and is given by

$$
\kappa_{\alpha}=\int_{m_{L}}^{m_{U}} \phi(m) \mathrm{d} m,
$$

where $m_{L}=0.1 M_{\odot}$ and $m_{U}=100 M_{\odot}$. The normalization of the IMF is the usual one (see Eq. 5).

The advantage of the DTD formulation is that we can test both theoretical and empirical Type Ia SN rates. These empirical rates can be approximated by analytical expressions such as that of Totani et al. (2008) which goes like $\operatorname{DTD}(t) \propto t^{-1}$. Another empirical Type Ia SN rate is that suggested by Mannucci et al. (2006): here, the Type Ia rate is bimodal and predicts that $50 \%$ of SNe Ia explode before 100 Myr and they are called prompt SNeIa, while the other $50 \%$ explode on timescales $>100 \mathrm{Myr}$ and they are called tardy SNeIa. It should be noted that the DTD of the empirical rate of Totani et al. (2008) well follows the theoretical DD rate of Greggio (2005).

In Fig. 4, we show several DTDs, both theoretical and empirical. For an extensive review about the empirically derived DTD functions for SNIa progenitors, we address the reader to Maoz and Mannucci (2012); in this paper, they conclude that a variety of methods to derive the DTD converges on a DTD such as that of Totani et al. (2008), at least in the range of $1<\tau<10 \mathrm{Gyr}$, thus suggesting the DD scenario as the preferred one. However, we cannot exclude that the SD can also work, if the problems related to the accretion rate from the donors are solved, as discussed above, and it can therefore be consistent with the empirical one (see Kobayashi et al. 2020b). It has been shown that to reproduce the abundance patterns in the solar vicinity the best scenarios are the SD and the DD ones, which predict a low number of prompt SNeIa, as opposed for example to the Mannucci et al. (2006) model. In particular, in the SD and DD model the DTD function contains $<20 \%$ of prompt SNeIa. If the prompt SNeIa represent a fraction larger than 30\%, the agreement with the observed abundance patterns in the Milky Way is lost (Matteucci et al. 2006, 2009). In Fig. 4, one can see that the DTD for the DD scenario does not differ much from the one for the SD scenario of Matteucci and Recchi (2001). 


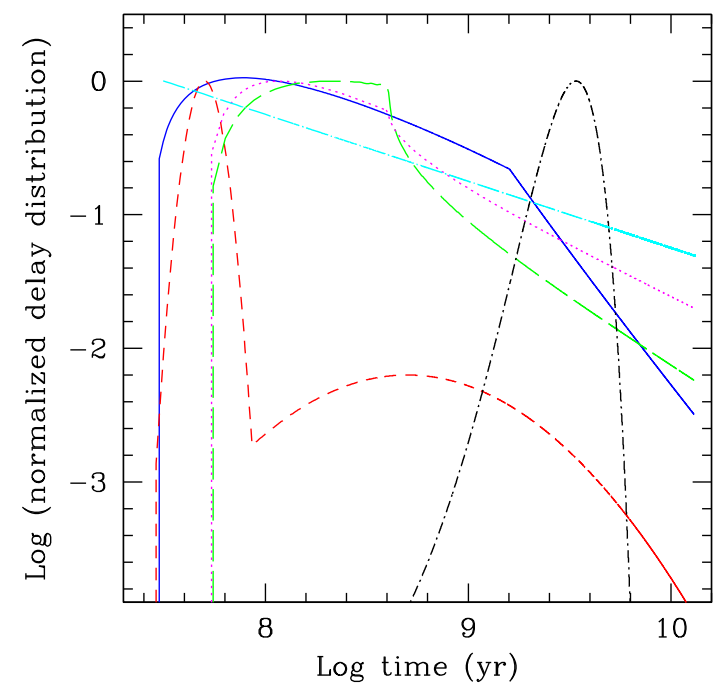

Fig. 4 Various DTD functions normalized to their own maximum value: the continuous blue line is the DTD for the SD scenario (Matteucci and Recchi 2001); the long dashed green line is the DTD of Greggio (2005) for the DD wide channel; the dotted magenta line is the DTD for the DD close channel of Greggio (2005); the dashed red line is the bimodal DTD of Mannucci et al. (2006); the short dashed-dotted black line is the DTD of Strolger et al. (2004) derived from the observed cosmic Type Ia SN rate and the cyan long dashed-dotted line is the DTD of Pritchet et al. (2008) with DTD $\propto t^{-0.5 \pm 0.2}$. Image reproduced with permission from Matteucci et al. (2009), copyright by ESO

\subsubsection{Novae}

Not only supernovae can enrich the ISM but also nova outbursts can eject newly formed elements, as discussed in Sect. 2. One way to compute the nova rate theoretically is to assume that it is proportional to the rate of formation of C-O WDs (D’Antona and Matteucci 1991)

$$
R_{\text {novae }}(t)=\delta \int_{0.8}^{8} \psi\left(t-\tau_{m_{2}}-\Delta t\right) \phi(m) \mathrm{d} m,
$$

where $\Delta t \sim 1$ Gyr (but it can be longer) is the delay time between the formation of the WD and the first nova outburst, and $\delta$ is a free parameter representing the fraction of WDs in the IMF belonging to binary systems giving rise to novae, and is normalized to reproduce the present time nova rate, after assuming that each nova system suffers roughly $10^{4}$ outbursts during its lifetime. The time $\tau_{m_{2}}$ is the lifetime of the secondary star (the less massive one) which determines the start of the mass accretion on the WD. In our Galaxy, the present time nova rate is estimated to be $\sim 20-25$ nova year ${ }^{-1}$ (see Della Valle and Izzo 2020). The value of $\delta$ derived by (Romano and Matteucci 2003) for the Milky Way is $\sim 0.01$. 


\subsubsection{Rate of MNS (kilonovae)}

The gravitational-wave event GW170817 (Abbott et al. 2017), identified also as kilonova AT2017gfo, has confirmed that MNS, or kilonovae, can be important producers of r-process elements (see Sect. 2). For this reason, it is important to compute the MNS rate in galaxies.

In Matteucci et al. (2014) the MNS rate has been derived by simply assuming that is proportional to the rate of formation of neutron stars.

In particular

$$
R_{\mathrm{MNS}}(t)=\alpha_{\mathrm{MNS}} \int_{8}^{50} \psi\left(t-\tau_{m}-\Delta_{\mathrm{MNS}}\right) \phi(m) \mathrm{d} m,
$$

with $\alpha_{\text {MNS }}$ being a free parameter indicating how many MNS systems are present in the mass range $9-50 M_{\odot}$, assumed to be the range of progenitors of neutron stars. I remind here that the upper mass in this range is largely uncertain depending upon mass loss, rotation, convection and other stellar physical parameters. The quantity $\Delta_{\text {MNS }}$ represents the time necessary for the neutron stars to merge, after emission of gravitational waves, and is very important to reproduce the abundance pattern of Eu/ Fe in the Milky Way, as we will see in the following sections. Matteucci et al. (2014) assumed a constant time delay of $1 \mathrm{Myr}, 10 \mathrm{Myr}$ and $100 \mathrm{Myr}$, and normalized the value of $\alpha_{\text {MNS }}$ to reproduce the present time observed rate of MNS in our Galaxy ( $\sim 80$ events $\mathrm{Myr}^{-1}$, as in Kalogera et al. 2004), and in such a case is derived to be $\alpha_{\text {MNS }}=0.018$.

Simonetti et al. (2019), where we address the reader for details, proposed a more refined calculation of the MNS rate based on a DTD function similar to what discussed for Type Ia SNe.

In particular, the suggested rate is

$$
R_{\mathrm{MNS}}(t)=k_{\alpha} \int_{\tau_{i}}^{\min \left(t, \tau_{x}\right)} \alpha_{\mathrm{MNS}} \psi(t-\tau) \operatorname{DTD}_{\mathrm{MNS}}(\tau) \mathrm{d} \tau,
$$

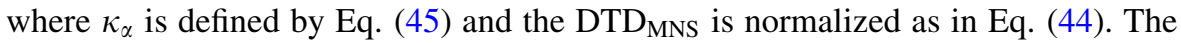
gravitational time delay $\Delta_{\text {MNS }}$ has been substituted by the DTD MNS $_{\text {function. This is }}$ a more detailed representation of the MNS rate and the DTD ${ }_{M N S}$ function depends upon the gravitational time delay and the distribution of the separations of the neutron stars, which is the most important parameter.

The distribution of separations is expressed as

$$
g(S) \propto S^{\beta},
$$

where $S$ is the initial separation between the two neutron stars and $\beta$ is a free parameter. Figure 5 shows the DTDs for four different values of $\beta=-1.5 ;-0.9 ; 0.0 ; 0.9$, and also DTDs $\propto t^{a}$ with $a=-2 ;-1.5 ;-1.0$. Côté et al. (2018) had explored first the two DTD functions with $a=-1.0$ and -1.5 . The

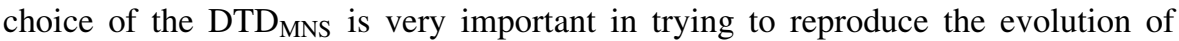
typical r-process elements, such as $\mathrm{Eu}$, as we will see in the next sections. 


\section{Historical model approaches}

In the past years, different approaches to the study of the chemical evolution of the Galaxy have been developed. We summarize them here:

- The serial approach

The formation of the Galaxy is modeled by a continuous accretion of gas during which the halo, thick and thin disks are formed in a temporal sequence (oneinfall model), as proposed by Chiosi (1980), Matteucci and Greggio (1986), Matteucci and Francois (1989) and Boissier and Prantzos (1999) among others, or by two different infall episodes forming the halo plus thick disk and the thin disk, respectively (the two-infall model, Chiappini et al. 1997), occurring in a temporal sequence. Micali et al. (2013) proposed a three-infall model, where the thick disk phase is treated separately from the halo and thin disk ones.

- The parallel approach

The formation of the Galaxy is modeled by different episodes of gas accretion occurring in parallel but at different rates (e.g., Pardi et al. 1995; Chiappini 2009; Grisoni et al. 2017).

- The stochastic approach

This approach is relative only to the formation of the stellar halo, when the gas mixing was probably not efficient and a large spread in the chemical abundances in stars is expected, reflecting the pollution by single supernovae. Among the

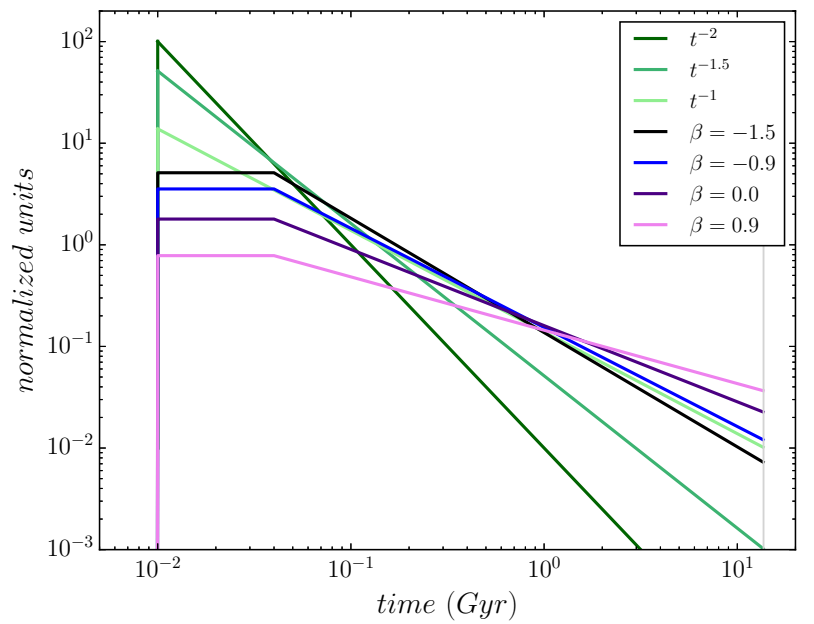

Fig. 5 Seven different DTD functions for describing the MNS. Three are described by simple power law functions (DTD $\propto t^{-a}$, with $a=-1.0,-1.5,-2.0$ ). The other four DTDs are the functions derived by Simonetti et al. (2019) for different values of the parameter $\beta$ which is the exponent of the function describing the initial separation of the two neutron stars $\left(g(S) \propto S^{\beta}\right.$ with $S$ being the separation). In such cases, three different phases are visible in the DTD functions: an initial null plateau, a plateau representing the close binary systems which promply merge and the tail for wide binary systems. The area under each curve is the same and equal to 1 . Image reproduced with permission from Simonetti et al. (2019), copyright by the authors 
papers of this kind I remind here as examples, those of Argast et al. (2000) and Cescutti (2008).

- The stellar accretion approach

Also this approach applies only to the stellar halo and assumes that it totally or partly formed by accretion of stars belonging to dwarf galaxies satellites of the Milky Way (e.g., Prantzos 2008b; De Lucia and Helmi 2008). This approach follows from the original suggestion of Searle and Zinn (1978), who suggested that the outer Galactic halo formed by merging of sub-galactic fragments over a long timescale. This assumption has mainly been explored by semi-analytical models of galaxy formation, and we will discuss them in more detail in the following sections.

\subsection{Serial approach and time delay model}

The serial approach was the first proposed: it assumes that one-infall episode has given rise first to the halo and then to the $\operatorname{disk}(\mathrm{s})$. In other words, the stars formed with ISM metallicity up to $[\mathrm{Fe} / \mathrm{H}]=-1.0$ dex belong to the halo, while the stars formed when $[\mathrm{Fe} / \mathrm{H}]>-1.0$ dex belong to the $\operatorname{disk}(\mathrm{s})$. These models predict a unique line as a function of time describing the average evolution of chemical abundances. An example of this approach is shown in Fig. 6, where the predicted $[\mathrm{O} / \mathrm{Fe}]$ vs. $[\mathrm{Fe} / \mathrm{H}]$ relations are compared with the data available at that time. The best model in Fig. 6 (model a) was obtained by assuming that $2 / 3$ of the $\mathrm{Fe}$ production comes from Type Ia SNe and 1/3 from CC-SNe (Matteucci and Greggio 1986), in excellent agreement with the recent nucleosynthesis yields by Kobayashi

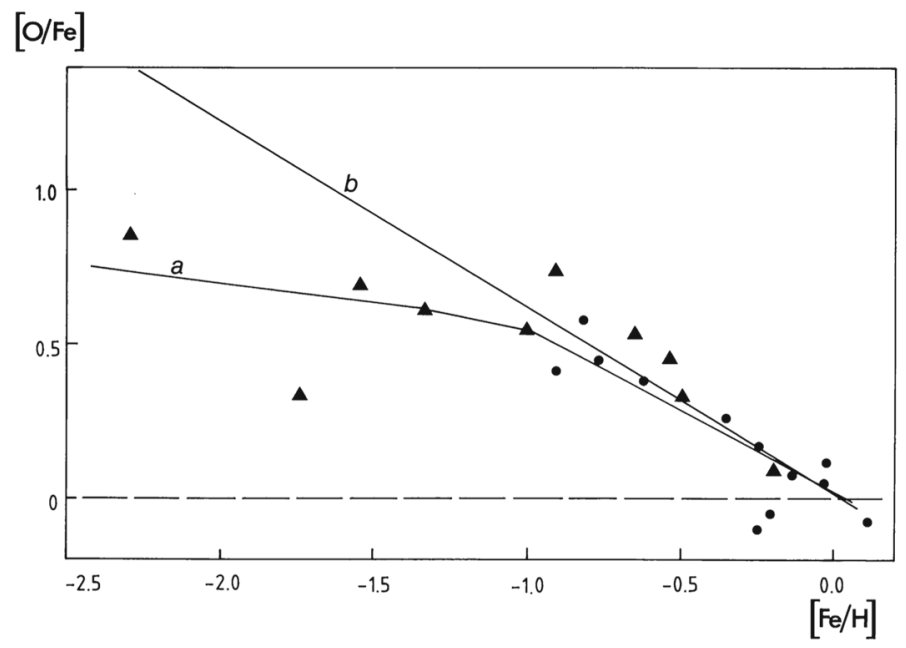

Fig. 6 Plot of $[\mathrm{O} / \mathrm{Fe}]$ vs. $[\mathrm{Fe} / \mathrm{H}]$ for solar neighbourhood stars compared with predictions from a oneinfall model (Matteucci and Greggio 1986). The line labelled a represents a model, where Fe is produced in both CC-SNe and SNe Ia, while the line labelled b shows the predictions of a model with $\mathrm{Fe}$ contributed only by SNe Ia. The data points are from Clegg et al. (1981) (circles) and Sneden et al. (1979) (triangles). Image reproduced with permission from Matteucci and Greggio (1986), copyright by ESO 
et al. (2020a). In fact, CC-SNe do produce some Fe and this fraction is important, because if one assumes that only Type Ia SNe do produce Fe, the agreement with data is lost (line b in Fig. 6). This figure is a very nice representation of the so-called time-delay model, namely, the interpretation of the $[\mathrm{O} / \mathrm{Fe}]$ vs. $[\mathrm{Fe} / \mathrm{H}]$ diagram: at low metallicity only $\mathrm{CC}-\mathrm{SNe}$ contribute to $\mathrm{Fe}$ enrichment and the $[\mathrm{O} / \mathrm{Fe}]$ ratio is roughly flat, since it reproduces the $(\mathrm{O} / \mathrm{Fe})$ production ratio in massive stars, while for $[\mathrm{Fe} / \mathrm{H}]>-1.0$ dex the SNe Ia start to be important in the Fe production and continue to be so up to the solar metallicity. From the best model in Fig. 6, one can extract the timescale for the formation of the Galactic halo: this timescale is simply the time at which the ISM reached the abundance $[\mathrm{Fe} / \mathrm{H}]=-1.0 \mathrm{dex}$, which roughly marks the maximum halo star metallicity. Matteucci and Greggio (1986) derived this timescale to be $\sim 1.0-1.5$ Gyr.

\subsection{Parallel approach}

A typical example of parallel approach can be found in Pardi et al. (1995), where the model predicts three separate curves describing the evolution of the abundances and abundance ratios as functions of time and metallicity. The formation of the three stellar components (halo, thick and thin disk) starts at the same time, but the bulk of star formation occurs at different times. Moreover, there is a connection in the formation of halo-thick disk and thick-thin disk: such connections are represented by the gas lost from the halo which forms the thick disk and the gas shed from the thick which goes to form the thin disk. In Fig. 7, we show the predictions of such a model in comparison with observations. The SFR during the halo and thick disk

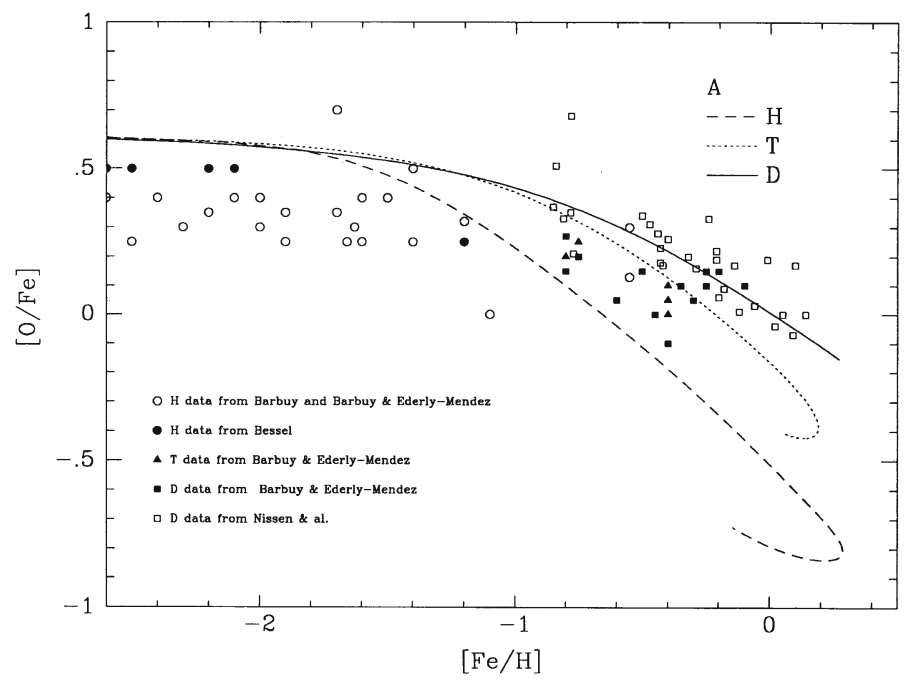

Fig. 7 Comparison between predicted $[\mathrm{O} / \mathrm{Fe}]$ vs. $[\mathrm{Fe} / \mathrm{H}]$ in the solar vicinity for the three Galactic components: halo (H), thick disk (D) and thin disk (T). One curve is predicted for each component and the three curves can represent the observed spread in the data. Data from Barbuy (1988); Barbuy and ErdelyiMendes (1989) and Nissen and Schuster (1991) as indicated by the legenda inside the figure. Image reproduced with permission from Pardi et al. (1995), copyright by AAS 
phases is assumed to be high and short, whereas in the thin disk is lower and more extended in time. It is worth noting that all the predicted curves cover the entire range of metallicities, but this is the evolution of the $[\mathrm{O} / \mathrm{Fe}]$ ratio in the ISM, which should be compared with the stellar abundance ratios, and therefore, it is important to know also how many stars have formed at each metallicity. This is described by the stellar MDF. For the halo and thick disk, the numbers of stars formed at metallicities larger than $[\mathrm{Fe} / \mathrm{H}]=-1.0$ dex for the halo, and -0.6 dex for the thick disk, are negligible. Therefore, Fig. 7 simply suggests that there could be a negligible number of halo and thick disk stars at high metallicity. However, this parallel model has a weak point: the connection in the formation of halo-thick disk and thick-thin disk is not realistic, since the thin disk is much more massive than the halo and thick disk and is hard to explain how it could have been formed from gas shed by the thick disk. Moreover, the parallel approach of Pardi et al. (1995) is at variance with the distribution of the stellar angular momentum per unit mass, indicating that the thick disk did not form out of gas shed by the halo (Wyse and Gilmore 1992).

In the modern view, the Galactic halo and thick disk might have formed by accretion of extragalactic gas and/or stellar systems and the thin disk out of extragalactic gas. As we will discuss later, in Chiappini et al. (1997), the two-infall model assumes two main independent gas infall episodes: during the first, the halo and the thick disk formed, while during the second the thin disk assembled on a longer timescale than the other two components.

In Grisoni et al. (2017), it was proposed a new version of the parallel model applied to the thick and thin disks. In particular, it was assumed that the formation by different gas infall episodes of the two disks started at the same time but it proceeded at different rates: the thick disk evolved faster with a high SFR and a short timescale of gas infall, whereas the thin disk assembled on a much longer timescale and moderate SFR. This approach could explain the double sequence of $[\mathrm{Mg} / \mathrm{Fe}]$ vs. $[\mathrm{Fe} / \mathrm{H}]$ found for the stars in the two disks, as it is evident in AMBRE data (de Laverny et al. 2013), and shown in Fig. 8. This "bimodality" in the $[\alpha / \mathrm{Fe}]$ ratios has been found also in APOGEE data (Nidever et al. 2014; Hayden et al. 2015) and it will be extensively discussed later.

\subsection{Stochastic approach}

In the framework of the stochastic approach (Travaglio et al. 2001; Suzuki and Yoshii 2001; Argast et al. 2004; Cescutti 2008; Cescutti et al. 2015; Haynes and Kobayashi 2019; van de Voort et al. 2015), a large spread is predicted for all the abundance ratios in halo stars, at least for $[\mathrm{Fe} / \mathrm{H}]<-3.0 \mathrm{dex}$. From an observational point of view, such a large spread is present only for s- and r-process elements, while it is much lower for other elements, such as $\alpha$-elements. As an example of that, we show in Fig. 9 the predictions of the inhomogeneous stochastic chemical model by Cescutti (2008). In particular, in Fig. 9, the $[\mathrm{Ba} / \mathrm{Fe}]$ vs. $[\mathrm{Fe} / \mathrm{H}]$ and the observational data (red triangles) show a large spread, which is well reproduced by the model. In addition, in this figure, we show the same model applied to $[\mathrm{Mg} / \mathrm{Fe}]$ vs. $[\mathrm{Fe} / \mathrm{H}]$ and in this case the observed and predicted spreads 


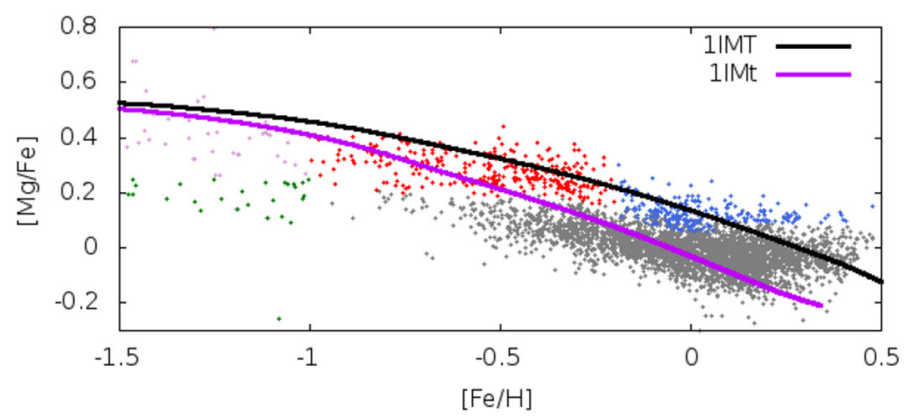

Fig. 8 Comparison between predicted $[\mathrm{Mg} / \mathrm{Fe}]$ vs. $[\mathrm{Fe} / \mathrm{H}]$ in the solar vicinity for the thick and thin disks.The black line represents the prediction for the thick disk while the magenta line for the thin disk. Here, the timescale for the formation of the thick disk is $\sim 1 \mathrm{Gyr}$, whereas that for the thin disk is $\sim 8$ Gyr. The red points are data relative to thick disk stars, while the grey points are thin disk stars (AMBRE data). The blue points represent stars whose origin is not well understood, they could either be high metallicity thick disk stars or stars migrated from the inner Galactic regions. Image reproduced with permission from Grisoni et al. (2017), copyright by the authors

are both smaller, indicating that if the observed spread is real there are some elements which are more affected by inhomogeneous mixing than others. The different spread observed in s-and r-process elements vs. $\alpha$-elements, has been interpreted by Cescutti (2008) as due to the fact that these two groups of elements are produced in different stellar mass ranges, coupled with a random birth of the stars. In particular, the mass range for production of neutron capture elements in
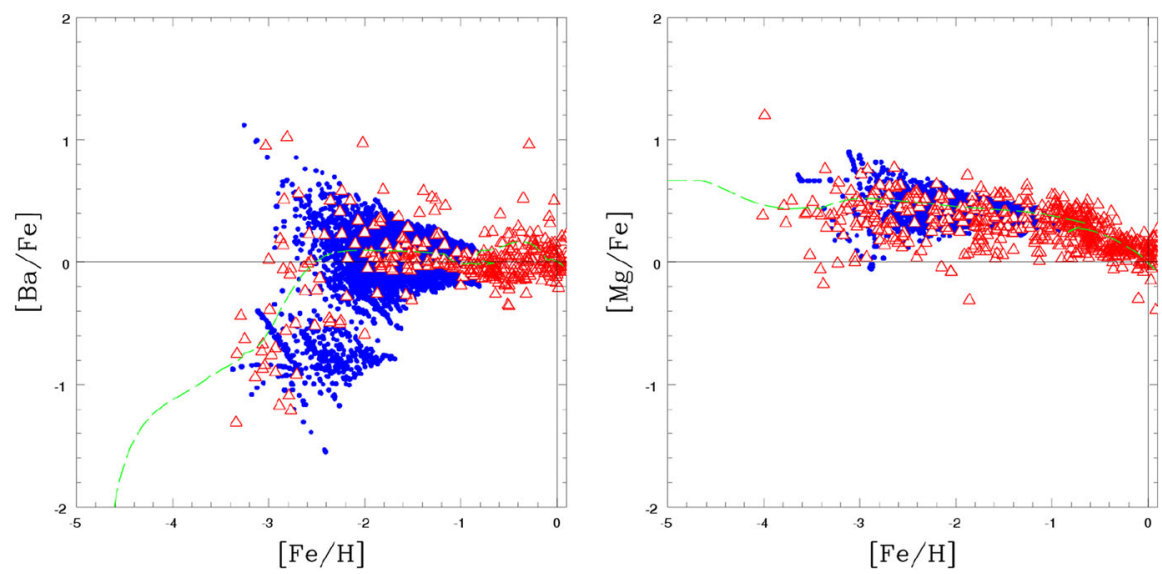

Fig. 9 Comparison between predicted $[\mathrm{Ba} / \mathrm{Fe}]$ vs. $[\mathrm{Fe} / \mathrm{H}]$ in the solar vicinity and observational data (left panel) and $[\mathrm{Mg} / \mathrm{Fe}]$ vs. $[\mathrm{Fe} / \mathrm{H}]$ (right panel). The blue points represent the predictions of an inhomogeneous stochastic chemical evolution model for the Galactic halo, while the red triangles are the observational points. The dashed green lines are the predicted average behaviours for the $[\mathrm{Ba} / \mathrm{Fe}]$ and $[\mathrm{Mg} / \mathrm{Fe}]$ ratios, as predicted by a homogeneous model (Cescutti et al. 2006). Image reproduced with permission from Cescutti (2008), copyright by ESO, where details on the model and references to the data can be found 
massive stars is restricted to $\sim 12-30 M_{\odot}$, whereas the mass range for production of $\alpha$-elements (e.g., $\mathrm{O}, \mathrm{Mg}$ ) is the whole range of massive stars. However, in subsequent works, the possibility of producing n-capture elements (such as $\mathrm{Ba}$ ) in the mass range $12-20 M_{\odot}$ has been rejected (Kobayashi et al. 2020a). In any case, the observed spread in s- and r-process elements at low metallicity is still a matter of debate (see also later).

\subsection{The classical two-infall model}

Chiappini et al. (1997) proposed the so-called two-infall model assuming two main independent but sequential in time infall episodes giving rise to the halo-thick disk and thin disk, respectively. The infall law proposed in that paper is

$$
A(R, t)=a(R) e^{-t / \tau_{H}}+b(R) e^{-\left(t-t_{\max }\right) / \tau_{D}(R)},
$$

where $a(R)$ and $b(R)$ are parameters, similar to $\Lambda$ in Eq. (11), and are derived by imposing that they reproduce the total surface mass density at the present time of the halo-thick disk and thin disk, respectively. The quantities $\tau_{H}$ and $\tau_{D}(R)$ are the timescale for the formation of the halo-thick disk and the thin disk, respectively. The quantity $t_{\max }$ is the time for the maximum infall onto the thin disk. In the best model of Chiappini et al. (1997, 2001), $t_{\max }=1 \mathrm{Gyr}$, whereas $\tau_{H}=0.8 \mathrm{Gyr}$ and $\tau_{D}(R)$ is assumed to vary with Galactocentric distance in such a way that the thin disk forms inside-out

$$
\tau_{D}(R)=1.033 R(\mathrm{kpc})-1.267 \mathrm{Gyr},
$$

where $\tau_{D}(8)=7 \mathrm{Gyr}$ is the timescale for the formation of the solar vicinity. The SFR was assumed to be a Schmidt-Kennicutt law with a dependence also on the total surface mass density

$$
\psi(R, t)=v(t)\left(\frac{\sigma(R, t)}{\sigma\left(R_{\odot}, t\right)}\right)^{2(k-1)}\left(\frac{\sigma\left(R, t_{\mathrm{G}}\right)}{\sigma(R, t)}\right)^{k-1} \sigma_{\mathrm{gas}}^{k}(R, t),
$$

where $v(t)$ is the efficiency of the star formation process, $\sigma(R, t)$ is the total surface mass density at a given radius $R$ and given time $t, \sigma\left(R_{\odot}, t\right)$ is the total surface mass density at the solar position and $\sigma_{\text {gas }}(R, t)$ is the gas surface density. The gas surface density exponent, $k=1.5$, in agreement with Kennicutt (1998) $(k=1.4 \pm 0.15)$. The efficiency of star formation was assumed to be $v_{H}=2 \mathrm{Gyr}^{-1}$ and $v_{D}=1 \mathrm{Gyr}^{-1}$ for the halo-thick and thin disk, respectively. The adopted IMF was that of Scalo (1986). They assumed also a gas density threshold in the star formation, namely, that star formation stops when $\sigma_{\text {gas }} \leq 7 M_{\odot} \mathrm{pc}^{-2}$ (Kennicutt 1989). Such a threshold has the effect of creating a gap of $<1 \mathrm{Gyr}$ in the SFR between the end of halo-thick disk and thin disk formation. Such a gap in the SFR should be visible in the abundance ratios vs. metallicity, as pointed out by Gratton et al. (2000) and Fuhrmann (1998). In particular, in Fig. 10, from Chiappini et al. (2001), we can see that the existence of a gap in star formation creates a loop in the curve describing the $[\mathrm{Fe} / \mathrm{O}]$ vs. $[\mathrm{O} / \mathrm{H}]$ relation in the solar vicinity, in particular, at $[\mathrm{O} / \mathrm{H}] \sim-0.5 \mathrm{dex}$, 


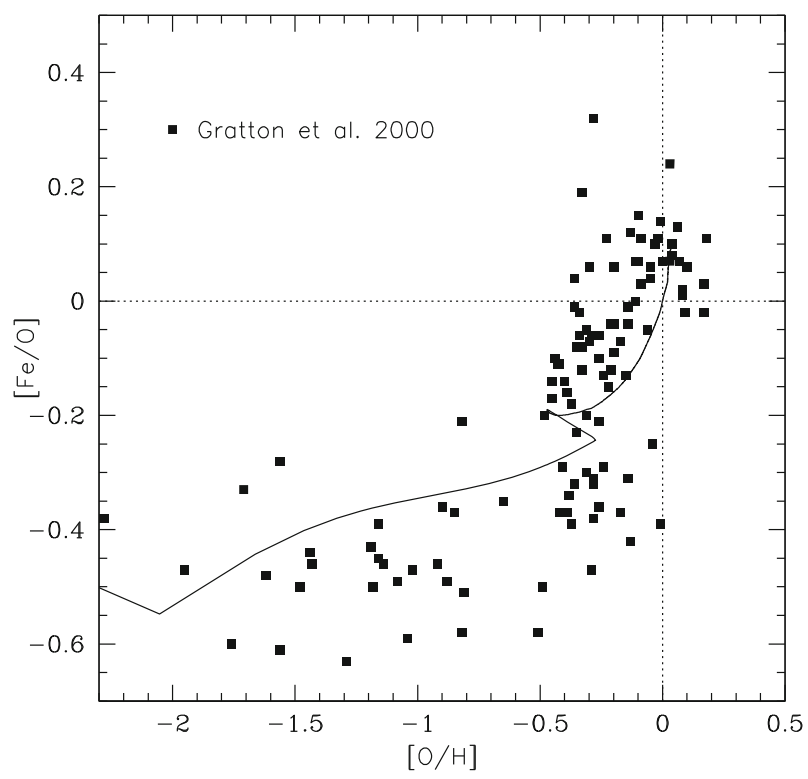

Fig. 10 Comparison between predicted and observed $[\mathrm{Fe} / \mathrm{O}]$ vs. $[\mathrm{O} / \mathrm{H}]$ in the solar vicinity. The points represent the observations of Gratton et al. (2000). As one can see, the data seem to show a lack of stars corresponding to $[\mathrm{O} / \mathrm{H}] \sim-0.5 \mathrm{dex}$. The model prediction shows a loop in correspondance of the same abundance, and this is due to the stop in the star formation produced by the assumption of a gas density threshold for star formation, as discussed in the text. Image reproduced with permission from Chiappini et al. (2001), copyright by AAS

corresponding roughly to $[\mathrm{Fe} / \mathrm{H}]=-1.0$ dex, this gap is visible also in the data, as discussed in Gratton et al. (2000). The behaviour of the [Fe/O] ratio in Fig. 10 is due to the fact that when star formation stops, $\mathrm{O}$ is no more produced, while $\mathrm{Fe}$ continues to be produced by Type Ia SNe. As a consequence of that, the $[\mathrm{Fe} / \mathrm{O}]$ ratio increases, while $[\mathrm{O} / \mathrm{H}]$ remains roughly constant, or slightly diminishes because of the infall related dilution, then when star formation starts again the $[\mathrm{Fe} / \mathrm{O}]$ ratio increases for increasing $[\mathrm{O} / \mathrm{H}]$. In Chiappini et al. (1997) and Chiappini et al. (2001), this effect is entirely due to the existence of a gas threshold which is not yet confirmed by observations; however, any event that could stop star formation at the end of the thick disk phase would predict the loop in the abundance ratios (e.g., $\alpha$ elements/Fe). In Fuhrmann (1998), a similar effect is observed for $\mathrm{Mg}$, as shown in Fig. 11 , where $[\mathrm{Fe} / \mathrm{Mg}]$ is plotted vs. $[\mathrm{Mg} / \mathrm{H}]$; here, the abrupt change of $[\mathrm{Fe} / \mathrm{Mg}]$ ratio at $[\mathrm{Mg} / \mathrm{H}] \sim-0.4$ dex shows a paucity of old stars and is indicative of a time when the enrichment of $\mathrm{Mg}$ from CC-SNe had stopped, while Fe enrichment continued, due to Type Ia SNe, even in absence of star formation.

This effect is present, although less clear, also in the plot $[\mathrm{Mg} / \mathrm{Fe}]$ vs. $[\mathrm{Fe} / \mathrm{H}]$ in Fig. 11. More recently, the gap in the SFR after the formation of the thick disk was suggested by Snaith et al. (2014), who analysed the [Si/Fe] vs. age plot based on a sample of F, G and K dwarfs in the solar vicinity from Adibekyan et al. (2012). According to their model, the Galaxy underwent an intense phase of star formation between 9 and 13 Gyr ago and this burst formed the thick disk. The estimated SFR 


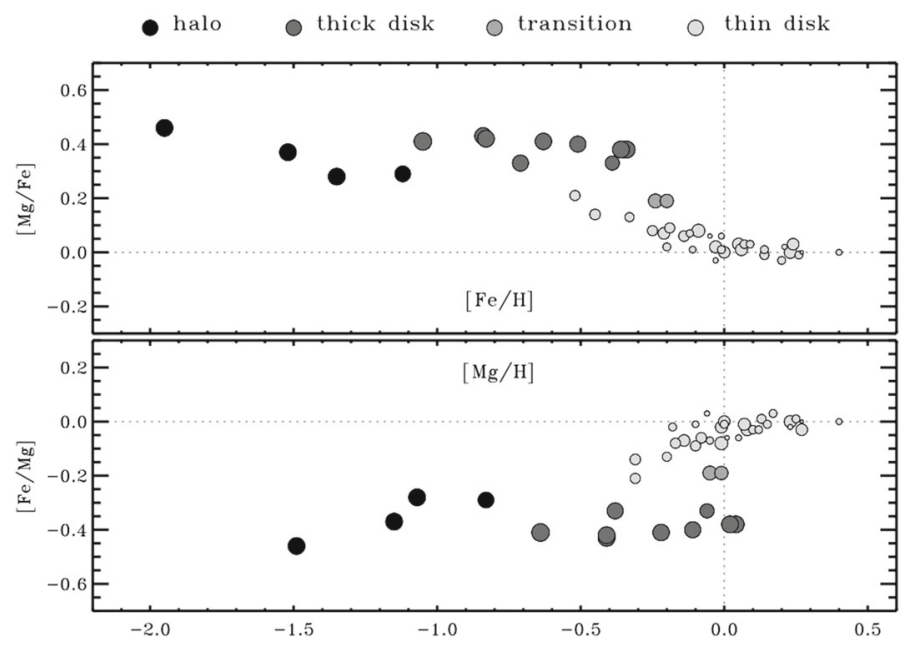

Fig. 11 Plot of the $[\mathrm{Mg} / \mathrm{Fe}]$ vs. $[\mathrm{Fe} / \mathrm{H}]$ for solar vicinity stars (upper panel) and $[\mathrm{Fe} / \mathrm{Mg}]$ vs. $[\mathrm{Mg} / \mathrm{H}]$ (lower panel). The stars belonging to halo, thick, thin disk and transition are indicated. Image reproduced with permission from Fuhrmann (1998), copyright by ESO

shows a dip at 8-9 Gyr ago and this corresponds to the end of the thick disk phase. Therefore, there are hints that a dip or a gap in the SFR occurred before the formation of the thin disk.

In summary, the two-infall model main results are: (i) the thin disk in the solar neighbourhood formed by infall of primordial gas on a long timescale (7-8 Gyr) and (ii) this timescale increases with Galactocentric distance giving rise to an inside-out formation of the disk, as already suggested by Matteucci and Francois (1989). (iii) The existence of a gas threshold in the SFR in the thin disk creates a gap in the star formation process between the formation of the two disks. This model can still reproduce the most recent data in the solar vicinity; in Fig. 12, we show the results of Grisoni et al. (2017) obtained with the two-infall model applied to the thick and thin disks. As one can see, the loop due to the stop in the star formation is visible, and is due to a gas density threshold of $7 M_{\odot} \mathrm{pc}^{-2}$. The timescale for accretion of the thick disk is $\tau_{\text {thick }}=0.1 \mathrm{Gyr}$ and for the thin disk is $\tau_{\text {thin }}=7 \mathrm{Gyr}$; the efficiency of star formation is $2 \mathrm{Gyr}^{-1}$ for the thick and $1 \mathrm{Gyr}^{-1}$ for the thin disk. The adopted IMF is that of Kroupa et al. (1993) for the solar vicinity. The model can reproduce the abundance patterns of thick and thin disk stars, but not the high $[\alpha / \mathrm{Fe}]$ at high metallicity blue stars (the same shown in Fig. 8). These stars, in the framework of the two-infall model can be explained only as stars migrated from the inner Galactic regions. Stellar migration represents a different and auxiliary paradigm for interpreting the abundance patterns in the solar vicinity, as we will see in the Sect. 8. Figure 13, displays a plot of the SFR vs. time, as predicted by the two-infall model of Grisoni et al. (2017), and it shows a clear gap in the star formation history, just before the formation of the thin disk. 


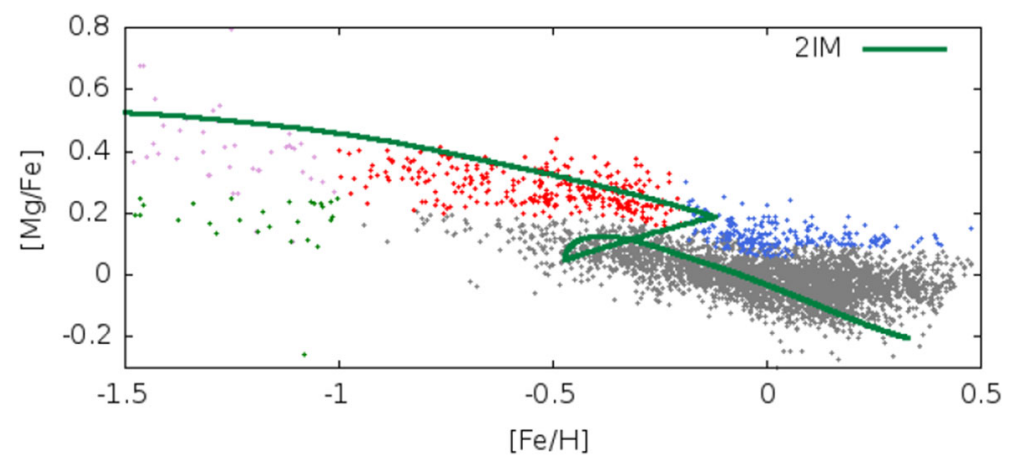

Fig. 12 Plot of $[\mathrm{Mg} / \mathrm{Fe}]$ vs. [Fe/H]: model vs. AMBRE data (as in Fig. 8). The model prediction (green line) is from the two-infall model of Grisoni et al. (2017). Image reproduced with permission from Grisoni et al. (2017), copyright by the authors

\subsubsection{A revised two-infall model and the bimodality in the $[\alpha / \mathrm{Fe}]$ ratios}

A revised two infall model, with the second infall occurring with a delay of $\sim 4.3$ Gyr relative to the previous one has been suggested by Spitoni et al. (2019). By means of this model, they were able to reproduce the bimodality in the $[\alpha / \mathrm{Fe}] \mathrm{vs}$. $[\mathrm{Fe} / \mathrm{H}]$ diagram $(\alpha=\mathrm{Mg}+\mathrm{Si})$ for thick and thin disk stars, as observed by APOKASC (APOGEE + Kepler Asteroseismology Science Consortium), as well as the stellar ages measured by asteroseismology, suggesting a large gap in age between thick and thin disk stars (Silva Aguirre et al. 2018). A similar conclusion has been reached by Noguchi (2018), who suggested two main episodes of cold gas infall,

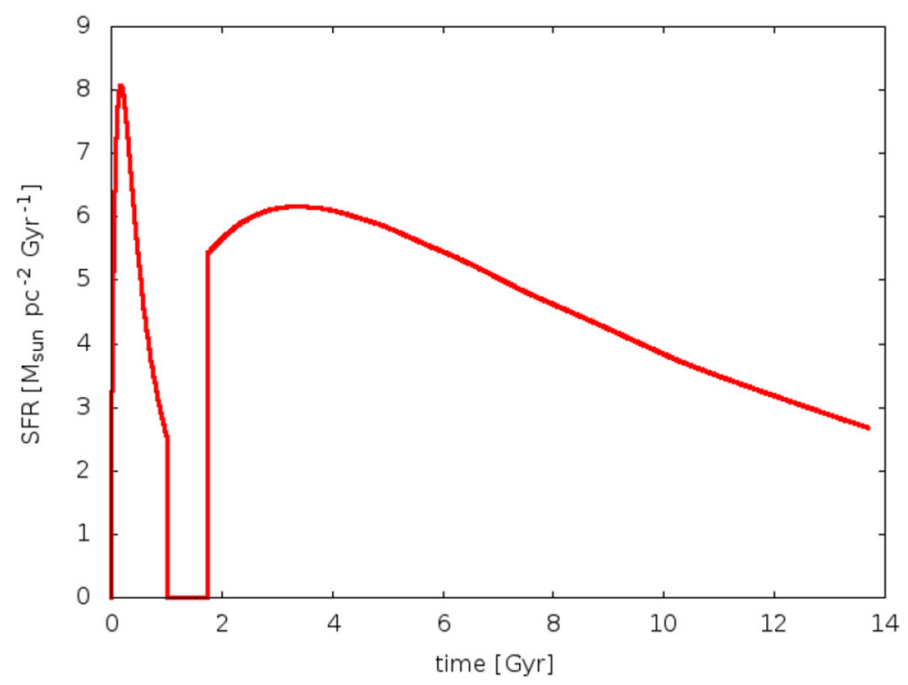

Fig. 13 SFR vs. time as predicted by the two-infall model. The gap in the SFR is evident and occurred over $12 \mathrm{Gyr}$ ago and lasted $\sim 1 \mathrm{Gyr}$, as in the original model of Chiappini et al. (1997). Image reproduced with permission from Grisoni et al. (2017), copyright by the authors 
with a hiatus in the star formation 6-7 Gyr ago, to reproduce the bimodality. In Fig. 14, we show the comparison between the predictions by Spitoni et al. (2019) and the APOKASC data. In Fig. 14, also displayed is the effect of varying the duration of the gap in star formation (indicated with $t_{\max }$ ): as one can see, a decrease in $t_{\max }$ produces a smaller loop starting at lower metallicities. Therefore, the flat $[\alpha /$ $\mathrm{Fe}]$ ratio observed in APOGEE thin disk stars can indeed be due to the strong infall episode which forms the thin disk and occurs at the end of the thick disk phase, a suggestion also made by Calura and Menci (2009), by means of a semi-analytical model of galaxy evolution. A different explanation for the bimodality in the $[\alpha / \mathrm{Fe}]$ distribution is provided by Buck (2020) and Sharma et al. (2020), suggesting that it can be explained by stellar migration (see later).

In conclusion, from all the previous discussion it seems that most of the data require separate gas accretion episodes to explain the abundances in the thick and thin disk stars, although the real delay between the two episodes, as well as the physical reason for such a delay, are still not well established.

\subsection{A three-infall model}

It is worth mentioning another approach to the formation and evolution of the Milky Way: the "three-infall model" by Micali et al. (2013). In this model, halo, thick and thin disk were formed by means of three different infall episodes separated by periods of quiescent star formation. Therefore, a continuous and not parallel evolution, similar to the two-infall model but with the thick disk well separated from the halo and thin disk. Thresholds in the gas density for star formation are also present in this model (4, 5 and $7 M_{\odot} \mathrm{pc}^{-2}$ for halo, thick and thin disk, respectively). By fitting of the solar vicinity $[\mathrm{X} / \mathrm{Fe}]$ vs. $[\mathrm{Fe} / \mathrm{H}]$ diagrams, they suggested that the halo formed on a timescale of $0.2 \mathrm{Gyr}$, the thick disk of $1.25 \mathrm{Gyr}$ and the thin disk, at the solar ring, of 6 Gyr. This model was also able to reproduce simultaneously the different observed stellar MDFs in the thick and thin disk, a constraint not always considered in chemical models.

As one can see from Fig. 15, the three-infall model predicts a more pronounced thick disk phase (black line) and shorter periods of quenched star formation between halo and thick and thick and thin disks, relative to the two-infall model.

\section{The evolution of heavy elements in halo and disks}

Numerical chemical evolution models are able to follow in detail the evolution of the abundances of a large number of chemical elements, both light ( $\mathrm{He}, \mathrm{D}, \mathrm{Li}, \mathrm{Be}$ and $\mathrm{B}$ ) and heavy $(\mathrm{C}, \mathrm{N}, \mathrm{O}, \alpha$-elements, Fe-peak elements, s- and $\mathrm{r}$ - process elements).

Here, we will summarize the results obtained in the last 10 years about the evolution of heavy elements. In Fig. 16, we report the results of Romano et al. (2010); these authors showed the abundances predicted by means of the two-infall model and two different sets of stellar yields, which are the most important parameters in galactic chemical evolution (see Côté et al. 2018). From the 

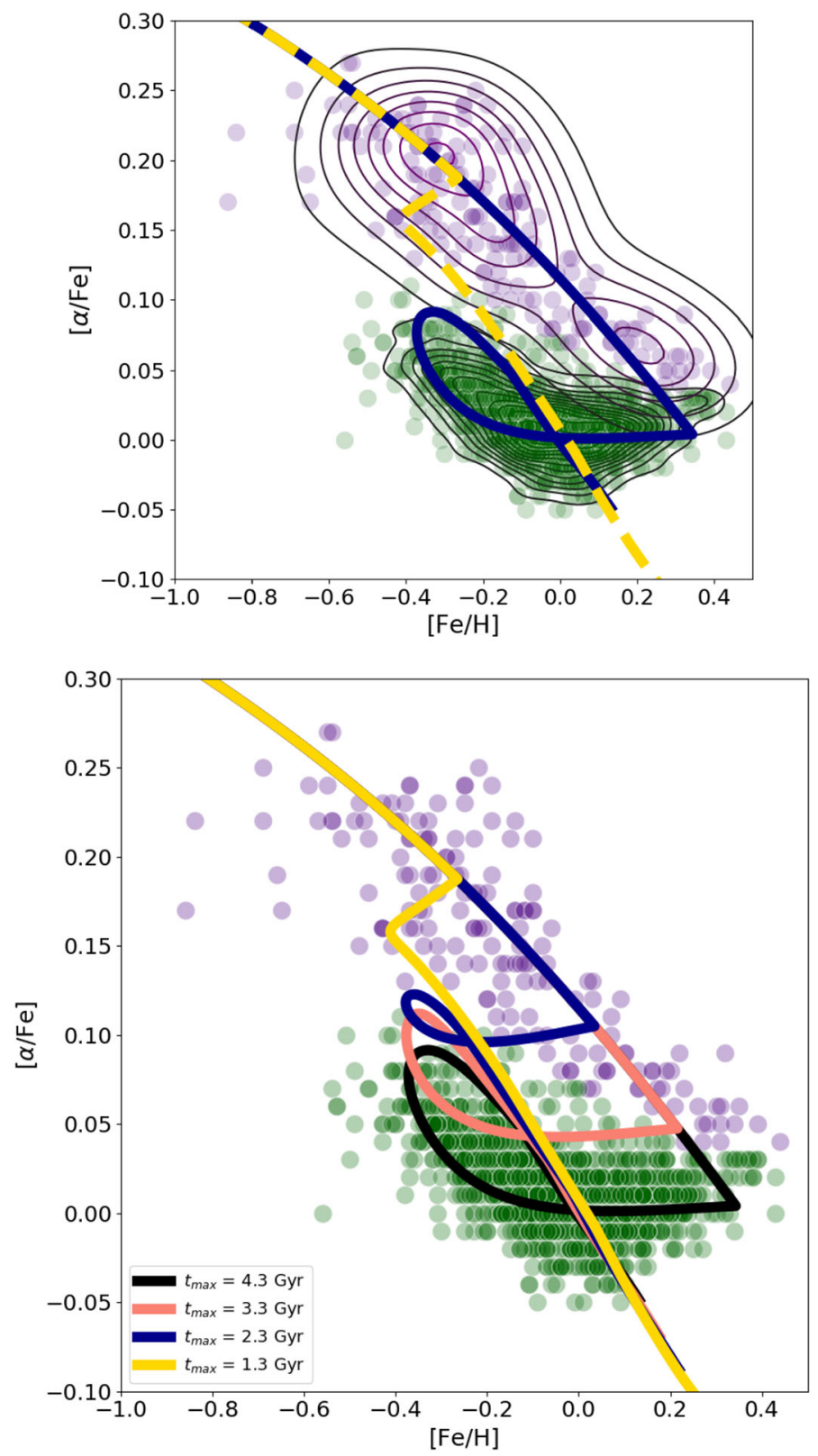

Fig. 14 Upper panel: comparison between predicted $[\alpha / \mathrm{Fe}]$ vs. $[\mathrm{Fe} / \mathrm{H}]$ in the solar vicinity and observational data by APOKASC (the purple filled circles are the observed "high- $\alpha$ stars" and green filled circles are the "low- $\alpha$ stars". The black-purple and black-green contour lines enclose the observed high- $\alpha$ and low $\alpha$ stars, respectively. The blue line is the prediction of Spitoni et al. (2019), while the yellow dashed line represents the prediction of the classical two-infall model. Lower panel: the effect of changing the timescale of the gap in the star formation $\left(t_{\max }\right)$ between the thick and thin disk. Image reproduced with permission from Spitoni et al. (2019) 


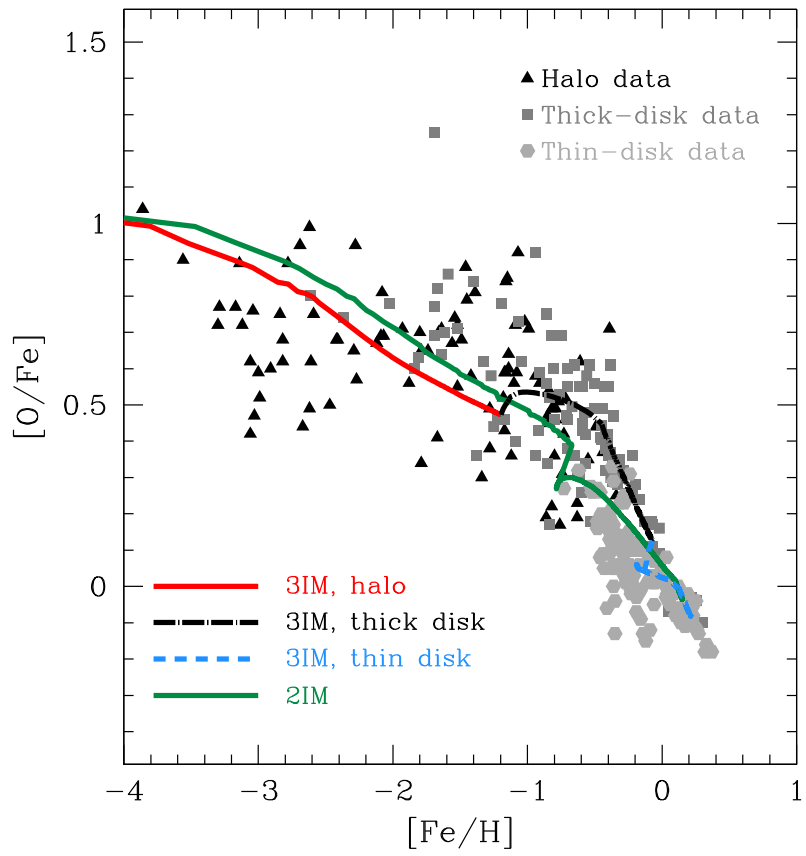

Fig. 15 Plot of $[\mathrm{O} / \mathrm{Fe}]$ vs. [Fe/H]: model predictions from the three-infall model (labelled 3IM, red, black and blue lines) and from the two-infall model (labelled 2IM, green line) compared to observations. References to the data can be found in Micali et al. (2013). Image reproduced with permission from Micali et al. (2013), copyright by the authors

comparison between model predictions and observations, it is evident that one set of yields should be preferred to reproduce the observed abundance patterns, as well as the solar abundances. However, some elements (in particular K, V, Ti and Sc) cannot be reproduced by any set of yields. The best yields suggested by Romano et al. (2010) include the results of Kobayashi et al. (2006) for nucleosynthesis in massive stars including hypernovae, but for $\mathrm{C}, \mathrm{N}, \mathrm{O}$ the yields with mass loss and rotation from the Geneva group are preferred. The yields of Karakas (2010) are suggested for low and intermediate mass stars and those of Iwamoto et al. (1999) for Type Ia SNe. They also suggested that the lack of agreement with some chemical species can be improved by including and improving the treatment of processes such as hot bottom burning in intermediate mass stars and stellar rotation in nucleosynthesis studies.

Prantzos et al. (2018) presented a study similar to that of Romano et al. (2010), with a one-infall Galactic model, where recent yields for low and intermediate mass stars depending on metallicity, as well as yields of massive stars including the combined effect of metallicity, mass loss and rotation, are adopted. In particular, for low and intermediate mass stars they adopted yields from the FRUITY database, ${ }^{2}$ and the yields for massive stars of Limongi and Chieffi (2018) including rotation

\footnotetext{
$\overline{2 \text { http://fruity.oa-abruzzo.inaf.it/. }}$
} 


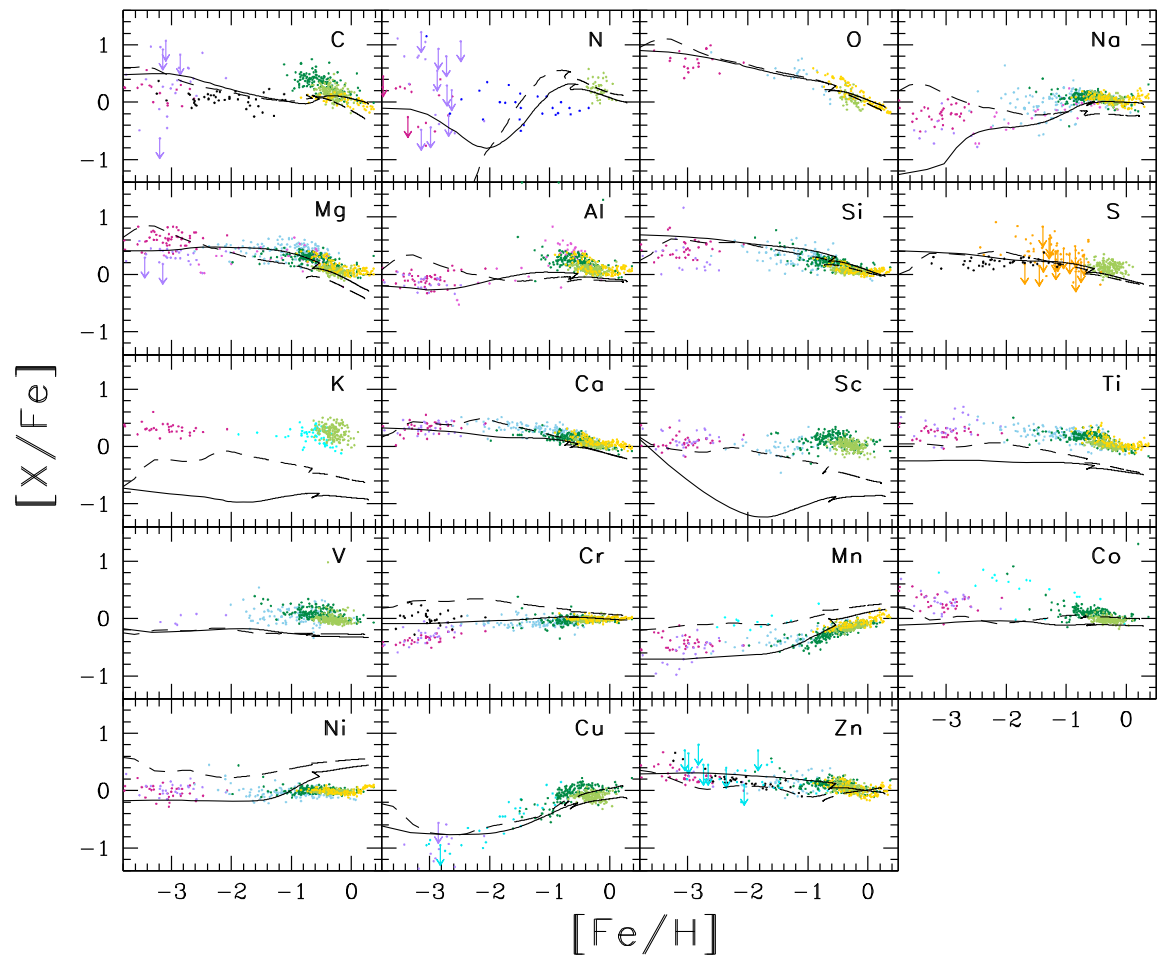

Fig. 16 Plot of the $[\mathrm{X} / \mathrm{Fe}]$ vs. $[\mathrm{Fe} / \mathrm{H}]$ relations for elements from $\mathrm{C}$ to $\mathrm{Zn}$. The solid curves represent the predictions of Model 15 of Romano et al. (2010), assuming the set of yields described in the text and considered as the best one (continuous lines). We note that this set of yields includes hypernovae. The dashed lines refer to a set of yields including those of Woosley and Weaver (1995) for massive stars, without mass loss and rotation, and those by van den Hoek and Groenewegen (1997) for low and intermediate mass stars. Some elements, such as V, K, Ti and Sc cannot be reproduced by any set of yields. The data are from a large compilation made by Romano et al. (2010), where the reader can find the references. Image reproduced with permission from Romano et al. (2010), copyright by ESO

and mass loss. In Fig. 17, we show the results of Prantzos et al. (2018) for a large number of heavy elements: two sets of theoretical curves are shown and they are representative of models with rotating and non-rotating massive stars, respectively. The evolution of most of the $\alpha$-elements is fairly well reproduced, as in Romano et al. (2010). The elements $\mathrm{Ne}$ and Ar, for which no data are available, show the same typical behaviour of $\alpha$-elements. The inclusion of stellar rotation does not change the results for $\alpha$-elements (apart from a small increase of $\mathrm{C}$ and $\mathrm{O}$ at low metallicities). For what concerns $\mathrm{Mg}$, the adopted yields underproduce $\mathrm{Mg}$, a problem common to previous sets of yields (e.g., Woosley and Weaver 1995), but not to the yields of Kobayashi et al. (2006) and Nomoto et al. (2013). Again, the elements $\mathrm{K}, \mathrm{Sc}$, Ti and V are not well reproduced by the adopted yields. However, the inclusion of rotation improves slightly the agreement with the abundances of $\mathrm{K}$ and $\mathrm{Sc}$ at low metallicities. The yields fail in reproducing the evolution of $\mathrm{Zn}$, a 


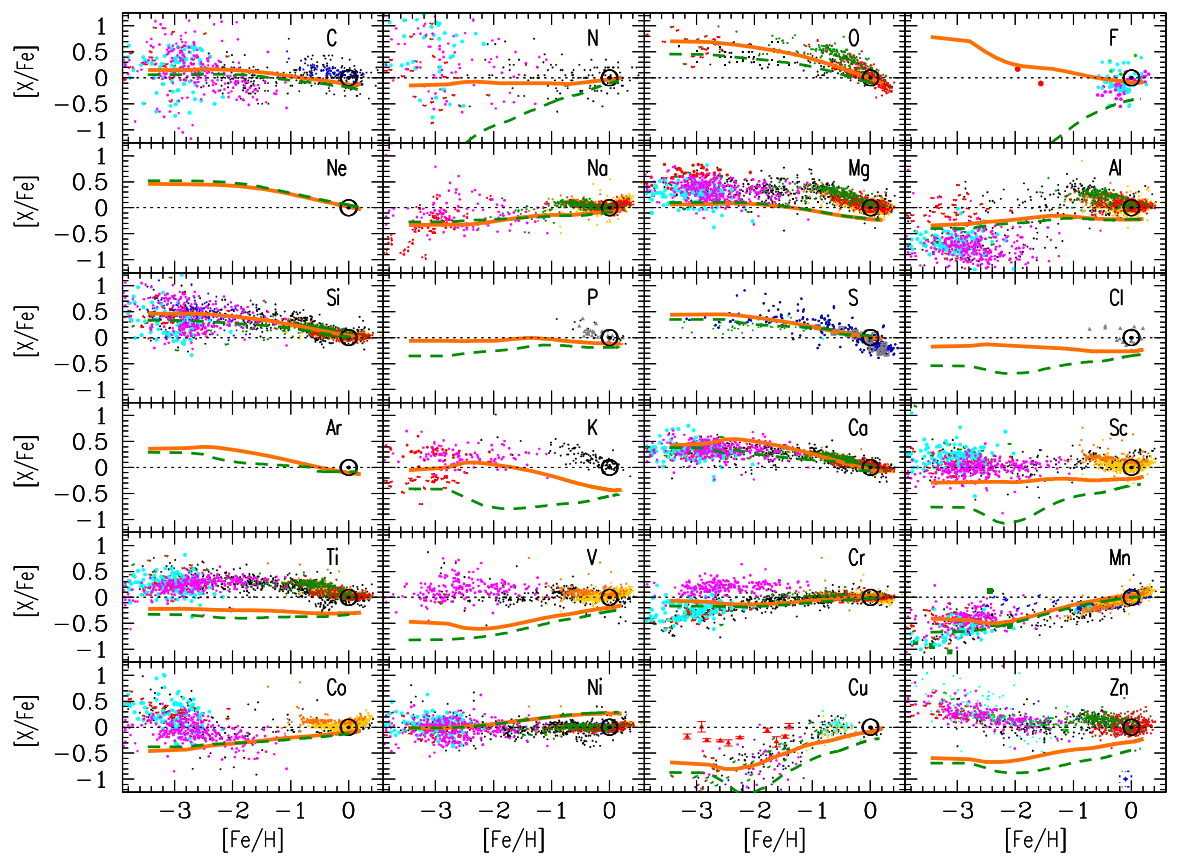

Fig. 17 Comparison between observed and predicted $[\mathrm{X} / \mathrm{Fe}]$ vs. $[\mathrm{Fe} / \mathrm{H}]$ relations by Prantzos et al. (2018) who adopt two sets of stellar yields, one with rotating massive stars (continuous lines) and the other with non-rotating massive stars (dashed lines). The adopted yields are described in the text and do not include hypernovae. Image reproduced with permission from Prantzos et al. (2018), copyright by the authors

feature shared by the yields of Woosley and Weaver (1995) and Kobayashi et al. (2006). The Fe-peak elements ( $\mathrm{Cr}, \mathrm{Mn}$, and $\mathrm{Ni}$ ) are largely produced by Type Ia $\mathrm{SNe}$, except for Co which is mainly produced in hypernovae. The increase of the $[\mathrm{Mn} / \mathrm{Fe}]$ here is, therefore, due only to the metallicity dependence of Mn yields in massive stars, otherwise Mn should evolve in lockstep with Fe. Cescutti et al. (2008) proposed instead that the secondary-like behaviour of Mn could be explained if the yields from SNe Ia have a dependence on metallicity, although such a feature is not present in the yields commonly adopted for Type Ia SNe (Iwamoto et al. 1999; Kobayashi et al. 2020a). The case of Co is not yet clear: no yields are able to reproduce the increase of $[\mathrm{Co} / \mathrm{Fe}]$ for $[\mathrm{Fe} / \mathrm{H}]<-2.0 \mathrm{dex}$. On the other hand, the increase of $\mathrm{Ni}$ with metallicity in Prantzos et al. (2018) models is due to the $\mathrm{Ni}$ overproduction in the typical nucleosynthesis model adopted for Type Ia $\mathrm{SNe}$ (model W7 and W70 of Iwamoto et al. 1999). However, more recent nucleosynthesis studies (e.g., Kobayashi et al. 2020a) do not overproduce Ni.

A particular discussion should be reserved to ${ }^{14} \mathrm{~N}$, a typical secondary element that instead reveals a primary behavior at low metallicities; see Figs. 16 and 17, where the data show a roughly constant $[\mathrm{N} / \mathrm{Fe}]$ ratio. Actually, ${ }^{14} \mathrm{~N}$ shows some primary contribution also at solar metallicities, as already indicated by Matteucci and Tosi (1985) and Diaz and Tosi (1986), due to the N produced during the hot bottom burning phase of intermediate mass stars. If $\mathrm{N}$ were produced as a typical 
secondary elements in massive stars, the $[\mathrm{N} / \mathrm{Fe}]$ ratio should increase with $[\mathrm{Fe} / \mathrm{H}]$. These facts suggest that $\mathrm{N}$ is produced as a primary element in massive stars, as first suggested by Matteucci (1986), and rotating massive stars can indeed produce primary N (Meynet and Maeder 2002; Chiappini et al. 2006; Kobayashi et al. 2011; Limongi and Chieffi 2018), as shown clearly in Fig. 17.

The evolution of the ${ }^{12} \mathrm{C}$ abundance is still a matter of debate. For many years, it was believed that ${ }^{12} \mathrm{C}$ originates from low and intermediate mass stars, but with the consideration of mass loss and rotation in massive stars, it appeared that massive stars can be the dominant source of carbon. The most recent observational data, showing an overabundance of $\mathrm{C}$ relative to $\mathrm{Fe}$, seem to confirm the massive star origin of ${ }^{12} \mathrm{C}$, as discussed by Romano et al. (2020).

\subsection{Very heavy elements: s- and r-processes}

The s- and r-process elements deserve a separate treatment, first of all, because unlike all the other heavy elements their abundances show a very large spread in halo stars, and because most of them can be produced partly as s- and partly as r-process elements.

\subsection{1 s-process elements}

We remind that s-process elements are formed by slow neutron capture on heavy seeds (e.g., Fe), relative to the timescale of the $\beta$-decay in nuclei. The r-process elements instead derive from rapid neutron capture. The s-process elements, such as $\mathrm{Sr}, \mathrm{Y}, \mathrm{Zr}$ (first peak), $\mathrm{Ba}, \mathrm{La}, \mathrm{Ce}, \mathrm{Nd}, \mathrm{Sm}$ (second peak) and $\mathrm{Pb}$ (third peak), are mainly produced during He-shell burning in low mass stars (1-3 $M_{\odot}$, see Busso et al. 1999; Cristallo et al. 2009, 2011; Karakas and Lugaro 2016), but they are also produced during He-core burning in massive stars $\left(M \geq 10 M_{\odot}\right.$ ) (Baraffe et al. 1992). Various authors have computed the chemical evolution of s-process elements (e.g., Travaglio et al. 1999; Cescutti et al. 2006; Cescutti and Chiappini 2014; Cescutti et al. 2013, 2015; Rizzuti et al. 2019). In particular, Travaglio et al. (1999) and Cescutti et al. (2006) suggested that s-process elements, such as Ba, are mainly produced by AGB stars but partly also by massive stars. Cescutti and Chiappini (2014) suggested that magneto-rotationally driven SNe (Winteler et al. 2012) can represent a promising source of s-process elements in the early Galaxy and that part of the production of Sr, Ba and Y most likely came from spinstars (e.g., Pignatari et al. 2008; Frischknecht et al. 2012; Limongi and Chieffi 2018). On the other hand, Kobayashi et al. (2020a) concluded that the first-peak elements come from electroncapture $\mathrm{SNe}$, while the magneto-rotationally driven $\mathrm{SNe}$ are responsible for the production of r-process elements. The inhomogeneous model of Cescutti and Chiappini (2014) was also able to reproduce the observed large spread at low metallicity observed for these elements. We have already shown the typical behaviour of a s-process element in Fig. 9; where the plot of $[\mathrm{Ba} / \mathrm{Fe}]$ vs. $[\mathrm{Fe} / \mathrm{H}$, shows an initial increase at low metallicity followed by an almost constant behaviour for $[\mathrm{Fe} / \mathrm{H}]>-2.0$ dex. The behaviour at low metallicity is due to the 
fact that $\mathrm{Ba}$ is assumed to be produced partly as a r-process element from stars in the range $10-30 M_{\odot}$, and therefore, it appears already at low metallicities.

\subsection{2 r-process elements}

For what concerns r-process elements ( $\mathrm{Eu}, \mathrm{Gd}, \mathrm{Dy}, \mathrm{Er}, \mathrm{Yb})$ they can be produced during explosive nucleosynthesis in CC-SNe (Cowan et al. 1991; Woosley et al. 1994; Wanajo et al. 2001), but still many uncertainties are present in the production of r-process elements by $\mathrm{CC}-\mathrm{SNe}$, since there are too few neutrons during explosive nucleosynthesis to reproduce the solar chemical composition (Arcones et al. 2007). An alternative and more successfull source of $r$ - process elements is the merging of compact objects, such as neutron stars and black holes (Lattimer et al. 1977; Meyer 1989; Freiburghaus et al. 1999; Rosswog et al. 1999; Korobkin et al. 2012; Thielemann et al. 2018).

This has been confirmed by the gravitational event GW170817 (Abbott et al. 2017, due to MNS, where r-process elements have been detected (Evans et al. 2017; Pian et al. 2017; Tanvir et al. 2017; Troja et al. 2017). The Eu is easily observable in stellar atmospheres and is a pure r-process element; therefore, most chemical evolution models refer to this element. Matteucci et al. (2014) included nucleosynthesis from merging of compact objects in the two-infall model and followed in detail the evolution of the abundances of Eu and Fe in the Milky Way. Concerning $\mathrm{Eu}$, both CC-SNe and MNS were taken into account as Eu producers. The main conclusions in that paper can be summarized as follows: (i) if each merging event produces $\sim 3 \times 10^{-6} M_{\odot}$ of Eu and the time delay for merging, due to gravitationalwave emission, is constant and short ( $\sim 1 \mathrm{Myr}$ ) for all systems, and the progenitors of neutron stars (or black holes) giving rise to the merging lie in the range $9-50 M_{\odot}$, then MNS can be responsible of the entire Eu production in the solar vicinity; (ii) If, instead, the time delays for merging are longer, then also CC-SNe need to be considered as Eu producers to fit the observed $[\mathrm{Eu} / \mathrm{Fe}]$ vs. $[\mathrm{Fe} / \mathrm{H}]$ plot at low metallicities. In this case, the amount of Eu produced per merging event should be lower $\left(\sim 2 \times 10^{-6} M_{\odot}\right)$, but always in the range suggested by nucleosynthesis calculations $\left(10^{-7}-10^{-5} M_{\odot}\right.$, Korobkin et al. 2012) and at the lower extreme of the range suggested for GW170817. This conclusion was confirmed by other papers (Cescutti et al. 2015; Côté et al. 2018; Simonetti et al. 2019; Molero et al. 2021). In Fig. 18, we show the results of Matteucci et al. (2014) for $[\mathrm{Eu} / \mathrm{Fe}]$ vs. $[\mathrm{Fe} / \mathrm{H}]$ in the cases of only MNS as Eu producers and of both CC-SNe and MNS.

As we have said before, the observational data concerning Eu show a very large spread and the model of Matteucci et al. (2014) cannot reproduce such a spread but just the average behaviour of the $[\mathrm{Eu} / \mathrm{Fe}]$ ratio, since this model assumes instantaneous mixing approximation. To explain the spread, if real, one has to adopt an inhomogeneous model for the Galactic halo, as shown in several recent works (Cescutti et al. 2015; van de Voort et al. 2015; Shen et al. 2015; Komiya and Shigeyama 2016; Wehmeyer et al. 2019; Cavallo et al. 2021). An example of inhomogeneous model results for the Galactic halo are shown in Fig. 19, where the model of Cescutti et al. (2015) assumes both MNS and magneto-rotationally driven 

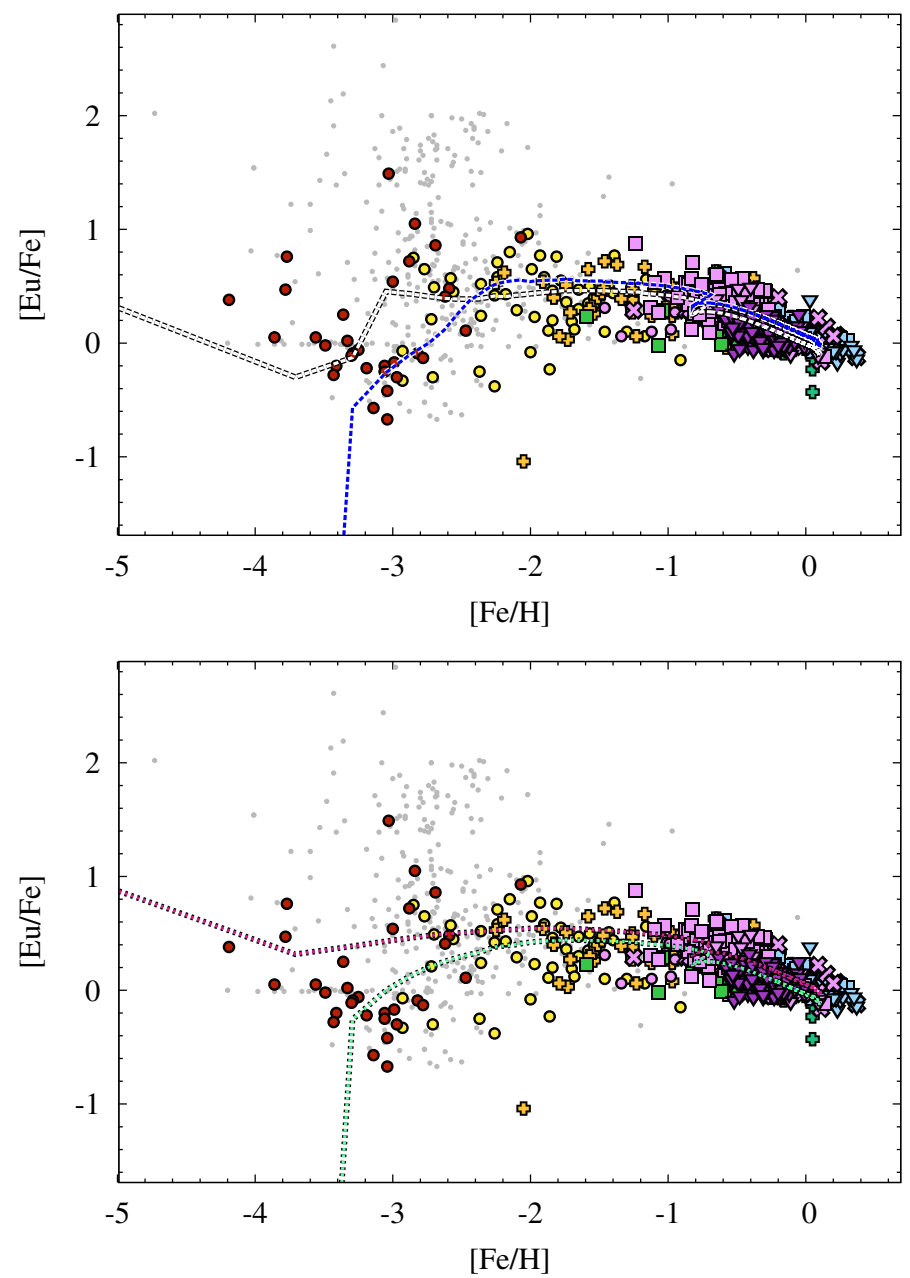

Fig. 18 Plot of the $[\mathrm{Eu} / \mathrm{Fe}]$ vs. $[\mathrm{Fe} / \mathrm{H}]$ for solar vicinity stars. The upper panel shows two models including both MNS and CC-SNe as Eu producers: they differ by the assumed delay time for MNS, $1 \mathrm{Myr}$ for the white curve and $10 \mathrm{Myr}$ for the blue curve and for the yields of Eu from massive stars; in both models the range of progenitors of neutron stars is $9-30 M_{\odot}$. The lower panel shows models, where only MNS produce Eu with a delay constant and equal to $1 \mathrm{Myr}$. The difference between the two curves are the mass range of neutron star progenitors. One model assumes a range $9-50 M_{\odot}$ (purple line), and the other a range of 9-30 $M_{\odot}$ (green line). The white line in the upper panel and the purple one in the lower panel are two examples of how we can fit the data either with CC-SNe plus MNS or only with MNS. Image reproduced with permission from Matteucci et al. (2014), copyright by the authors

$\mathrm{SNe}$ as Eu producers. As one can see, in both cases the observed spread is rather well reproduced. The need for introducing $\mathrm{CC}-\mathrm{SNe}$ as Eu producers arises from the fact that, in this way, one can adopt MNS delay times much longer than $1 \mathrm{Myr}$, in agreement with the GW170817 which occurred in an early type galaxy, where star formation had stopped several Gyr ago. Longer delay times are also required to fit the observed cosmic rate of short gamma-ray bursts (Ghirlanda et al. 2016), as 


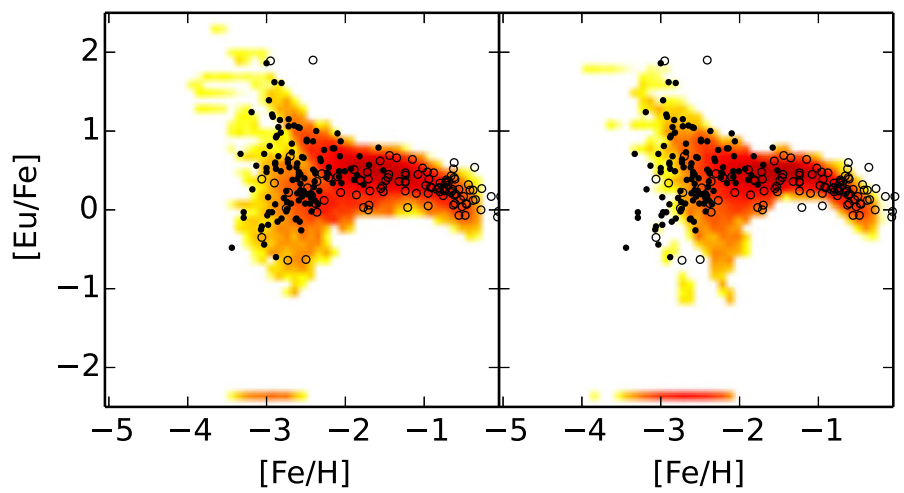

Fig. 19 Plot of the $[\mathrm{Eu} / \mathrm{Fe}]$ vs. $[\mathrm{Fe} / \mathrm{H}]$ for Galactic halo stars. The model predictions are indicated by the red and yellow areas, while the data are the black circles. The left panel shows a model with only MNS as Eu producers with a constant delay of $1 \mathrm{Myr}$, as in Matteucci et al. (2014). The right panel shows the model including MNS with fixed delay time of 100 Myr plus the contribution of magneto-rotationally driven SNe acting only for metallicities $Z<10^{-3}$. See Cescutti et al. (2015) for the references to the data. Image reproduced with permission from Cescutti et al. (2015), copyright by ESO

shown by several papers (Simonetti et al. 2019; Molero et al. 2021). A way to reconcile the observed $[\mathrm{Eu} / \mathrm{Fe}]$ ratios in the Milky Way, which requires a fast Eu production at early times, together with long coalescence timescales, could be that the fraction of systems giving rise to MNS decreases with time, as suggested by Simonetti et al. (2019). On the other hand, in some cosmological simulations the need of a short coalescence timescale to reproduce the $[\mathrm{Eu} / \mathrm{Fe}]$ at low metallicity seems not to be present, as shown by Shen et al. (2015), who adopted a minimum delay time of $100 \mathrm{Myr}$ and claimed to be able to predict Eu abundances different from zero also in stars of very low metallicity as well as the observed spread. This is clearly an effect of the stellar enrichment in cosmological simulations, but it is not clear from the paper how many stars with zero Eu abundance are predicted at very low metallicity and the predicted $[\mathrm{Eu} / \mathrm{Fe}]$ along the disk looks too flat compared to the data. Finally, Schönrich and Weinberg (2019) showed that with a DTD for MNS with a typical timescale of $150 \mathrm{Myr}$ they could reproduce the $[\mathrm{Eu} / \mathrm{Fe}]$ pattern in the Galaxy, if they allow for a 2-phase interstellar medium (hot and cold).

In conclusion, we still do not know whether merging of compact objects can be entirely responsible for the production of r-process elements.

\section{Abundance gradients along the thin disk}

It is well known that the abundance of metals decreases with Galactocentric distance along the thin disk. Negative abundance gradients are present also in external spirals and they represent an important tool to understand the formation mechanism of galactic disks. Negative abundance gradients of metals can be obtained if the star formation has been more efficient in the inner than in the outer regions of the disk. In the Milky Way, the SFR is indeed higher towards the Galactic center at the present time (Rana 1991; Stahler and Palla 2004). There are several 
ways to reproduce abundance gradients by means of chemical evolution models. One way is to assume an inside-out formation of the disk, as suggested originally by Larson (1976) and then by Matteucci and Francois (1989), Boissier and Prantzos (1999) and Pilkington et al. (2012): in this scenario, the disk forms by gas accretion occurring much faster in the inner than in the outer disk regions, thus creating a gradient in the SFR. This inside-out mechanism can be easily achieved by assuming a time scale of gas accretion increasing with Galactocentric distance in an exponential infall law of the type of Eq. (51). Other ways of obtaining and/or steepening a metal gradient are the presence of a threshold in the gas density for star formation, a star formation efficiency ( $v$ in Eqs. 2 and 52) decreasing with Galactocentric distance, and inwards radial gas flows. All these situations have been studied (e.g., Chiappini et al. 2001; Colavitti et al. 2009; Portinari and Chiosi 2000; Schönrich and Binney 2009; Spitoni and Matteucci 2011; Mott et al. 2013; Cescutti et al. 2007; Bilitewski and Schönrich 2012; Cavichia et al. 2014; Grisoni et al. 2018; Palla et al. 2020). Moreover, also an equidense infall rate has been suggested (e.g., Tosi 1982, 1988b). In Fig. 20, we report an example of predicted and observed abundance gradients: the models shown are all based on an inside-out formation of the thin disk plus other mechanisms, such as variable star formation efficiency and radial gas flows. In particular, radial gas flows should have a speed not exceeding $2-4 \mathrm{~km} / \mathrm{s}[(0.7 \mathrm{~km} / \mathrm{s}$ is the speed found in the cosmological simulation of Vincenzo and Kobayashi 2020)], otherwise the inward flows erase the gradient (Tinsley 1980), but this speed can be constant or variable (Spitoni and Matteucci 2011) or even vary with time (Palla et al. 2020). Radial gas flows were first studied by Mayor and Vigroux (1981) and Lacey and Fall (1985) and are a consequence of gas infall onto the disk.

As one can see from Fig. 20, the gradients derived from nebular data (HII regions and Planetary Nebulae (PNe), black points with error bars) are different from those derived from Cepheids and young open clusters (red points with error bars). This is probably due to an observational bias in at least one of the two observational techniques. The models are all based on the inside-out mechanism for the formation of the thin disk: it is clear from the figure that also other mechanisms, such as variable star formation efficiency and radial gas flows, should be included to properly reproduce the gradients.

\subsection{The $[\alpha / \mathrm{Fe}]$ ratio bimodality at different Galactocentric distances}

Following the study of Hayden et al. (2015), in Queiroz et al. (2020) a complete map of the $[\alpha / \mathrm{Fe}]$ ratios vs. $[\mathrm{Fe} / \mathrm{H}]$, extending from the bulge to the outer Galactic regions $\left(R_{G}>20 \mathrm{kpc}\right)$, has been presented. This map is based on high resolution spectroscopic data from APOGEE-2 survey Data Release 16 (DR16). These results show that the $[\alpha / \mathrm{Fe}]$ bimodality extends from the very inner Galactic regions to the outermost ones, suggesting that the bimodality is a clear property of the chemical enrichment of the Milky Way. These data are displayed in Figs. 21 and 22, where the diagram $[\alpha / \mathrm{Fe}]$ vs. $[\mathrm{Fe} / \mathrm{H}]$ is shown in $2 \mathrm{kpc}$ wide bins of the Galactocentric radius $R_{G}$. In these figures, is present also a subdivision in $z$ is present (the height 

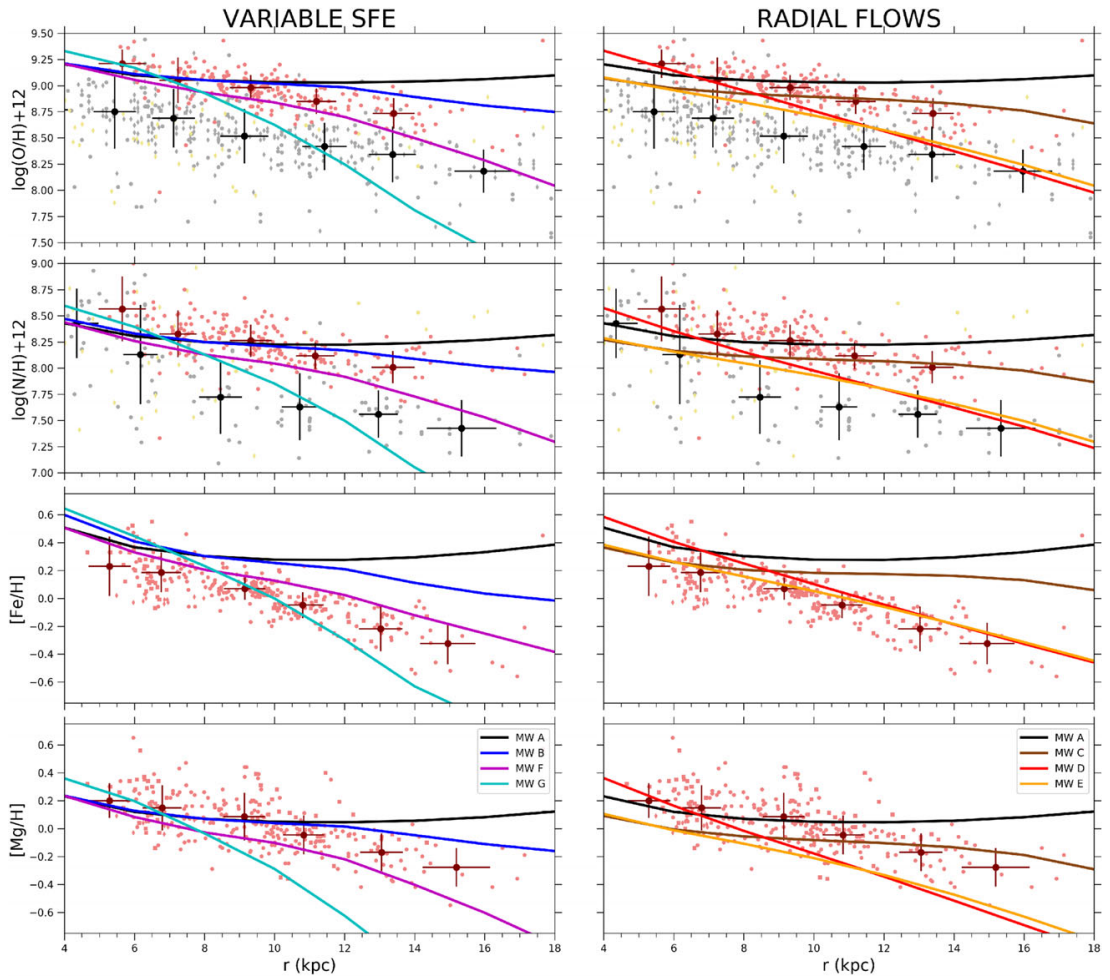

Fig. 20 Observed and predicted abundance gradients along the thin disk. Black points with error bars represents the HII regions and $\mathrm{PNe}$, while the red points with error bars refer to stars (Cepheids and young open clusters). Model A includes the inside-out mechanism but no radial flows and constant efficiency of star formation along the thin disk. Clearly it predicts too flat gradients. The models labeled B, F and G in the left panel include different prescriptions for a variable star formation efficiency, while models labeled $\mathrm{C}, \mathrm{D}$ and $\mathrm{E}$ in the right panel contain radial gas flows with different prescriptions for the flow speed. All the models include the inside-out formation mechanism. For details and references see Palla et al. (2020). Image reproduced with permission from Palla et al. (2020), copyright by the authors

above the Galactic plane). The interpretation of these data is very important to understand how the thick and thin disks formed.

As we have already discussed, in Spitoni et al. (2019), it has been suggested that a rather long period of quiescent star formation between the formation of the thick and thin disks can explain the observed $[\alpha / \mathrm{Fe}]$ bimodality in the solar vicinity data. In Figs. 21 and 22, it is evident that the low $[\alpha / \mathrm{Fe}]$ sequence moves towards lower $[\mathrm{Fe} / \mathrm{H}]$ values with Galactocentric distance. This is a sign of an inside-out formation of the thin disk: in fact, a longer accretion timescale for the thin disk means lower SFR and lower $[\alpha / \mathrm{Fe}]$ ratios, according to the time-delay model, as discussed in the previous sections. Concerning the innermost Galactic regions, the two sequences are less distinct than in the outer parts and probably an enriched infall to form the thin disk is required to reproduce it, as shown by Palla et al. (2020) and Spitoni et al. (2021) (see Fig. 23). Finally, a trend is present also with the height above the 


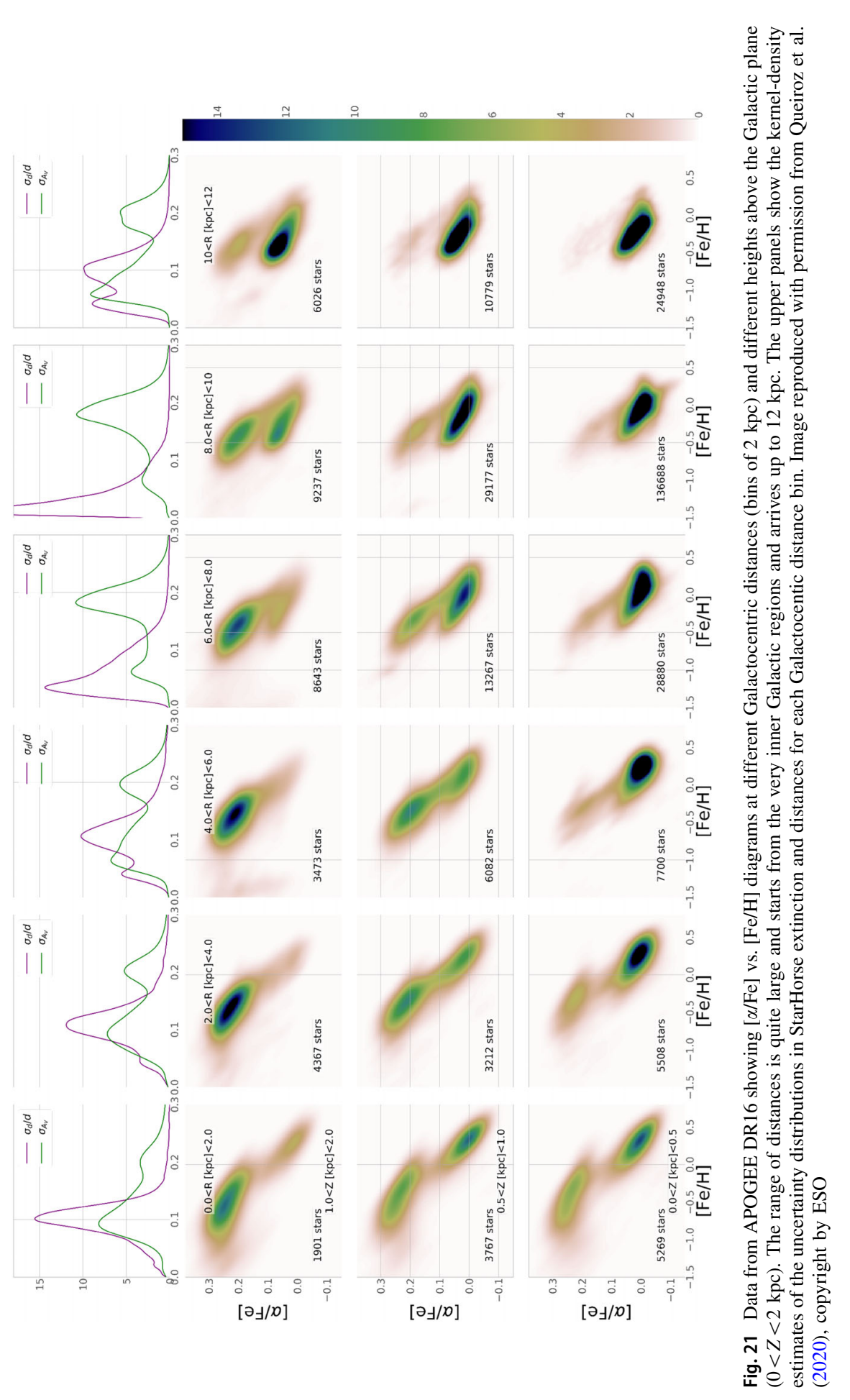




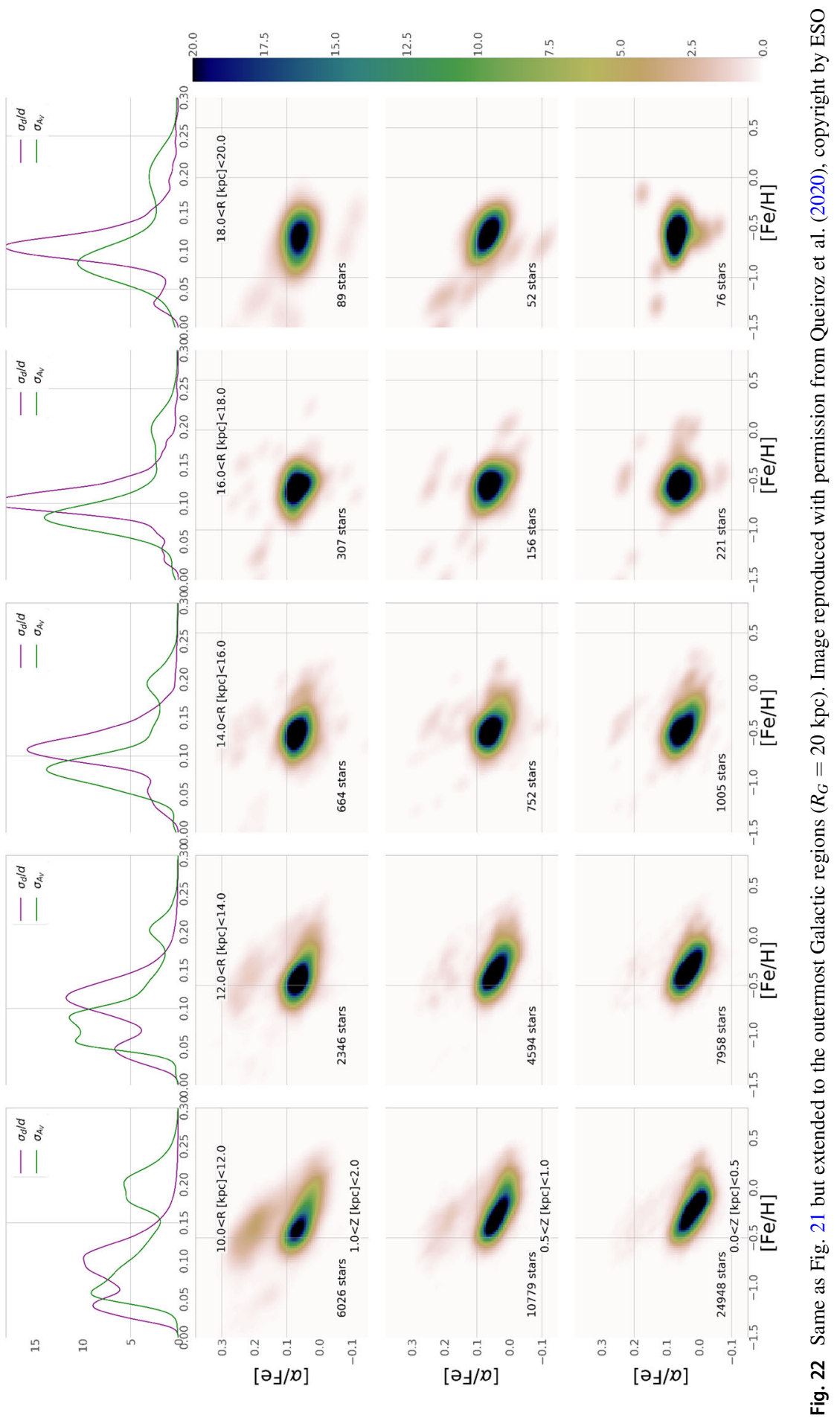




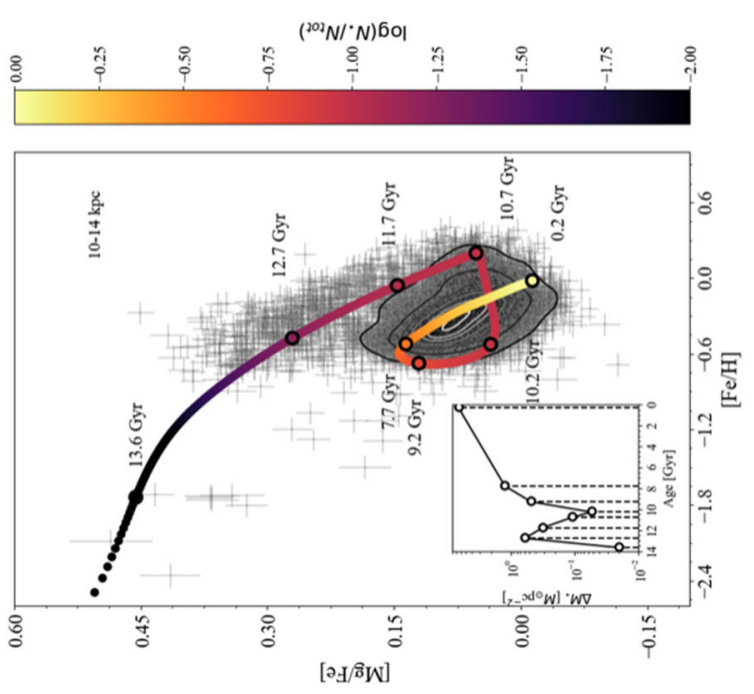

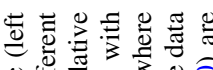

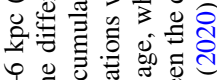

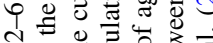

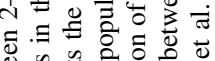

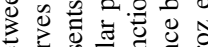

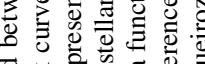
D.

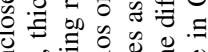

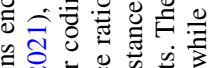

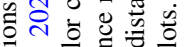

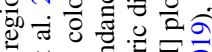
U.

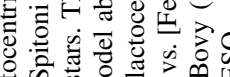

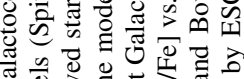

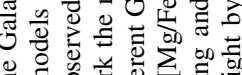

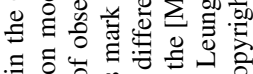
$\Xi$ 능

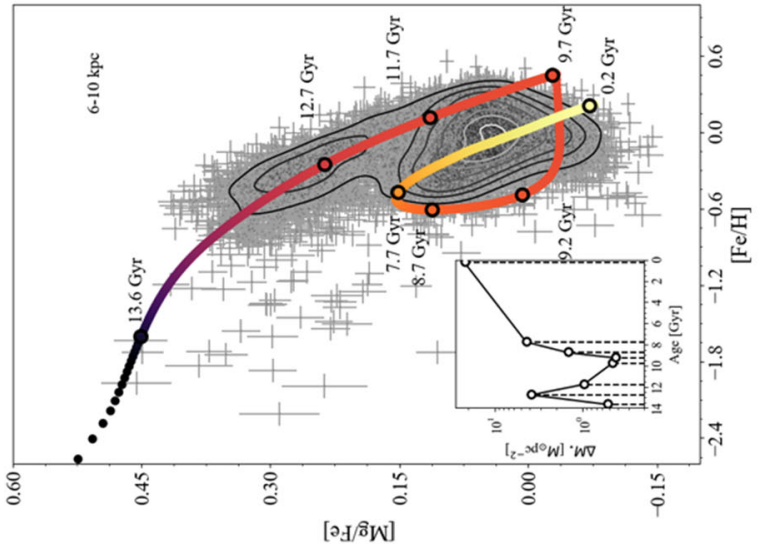

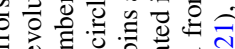
य व

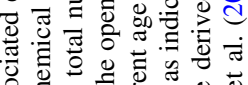

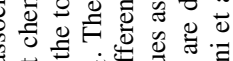

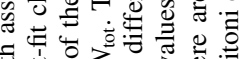

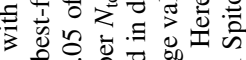

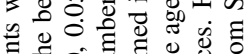

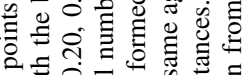
入.

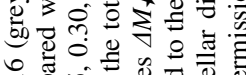

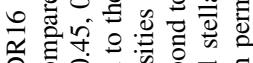

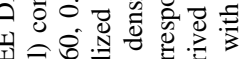

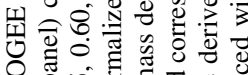

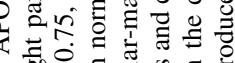
๘.000

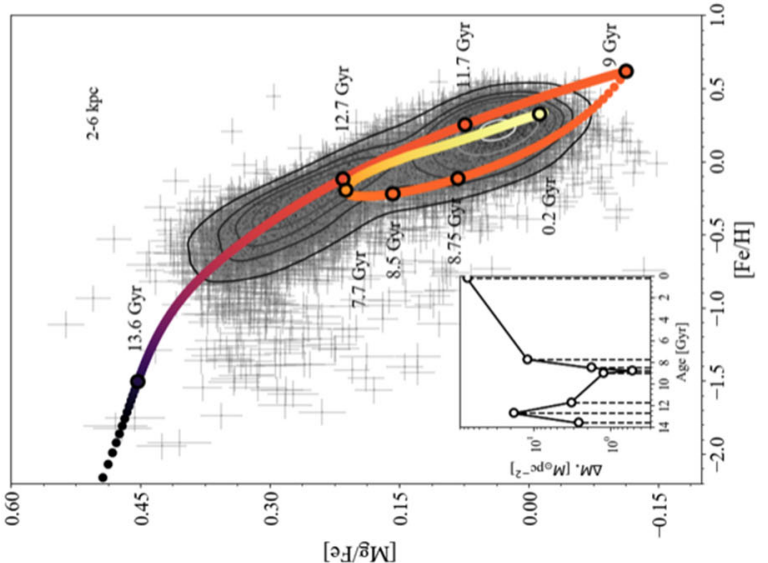

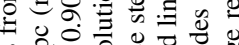
章

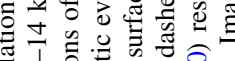

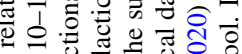

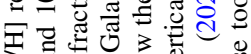

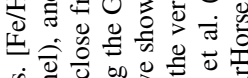

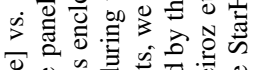

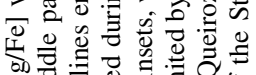

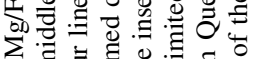

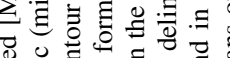

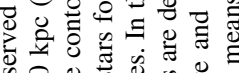

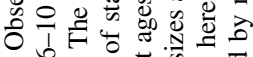
๗ิ)

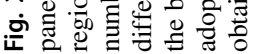


Galactic plane, and indicates that the high- $\alpha$ sequence is more pronounced at larger heights.

\section{A different paradigm: stellar migration}

Generally, in chemical evolution models it has been assumed that gas can move along the disk (radial gas flows) but not stars. Roškar et al. (2008), by means of a cosmological simulation for galaxy formation which included gas and stars, showed that stars tend to move outwards, while the gas move inwards, and this is caused by resonant scattering at corotation. This result confirmed an idea of Binney and Sellwood (2000) according to which the gas would participate in resonant scattering together with stars. The stellar migration can significantly alter our interpretation of the observational data, simply because the stars we observe at the solar ring might have formed at inner radii and then been scattered at the solar radius. It clearly depends on the entity of this phenomenon quantified by the percentage of stars which have not been formed in situ. A great deal of work on the stellar migration followed the paper of Roškar et al. (2008): in particular, Schönrich and Binney (2009) presented a chemical model including radial gas flows and stellar migration. The physical causes for stellar migration that they considered are: the change of angular momentum by either (i) scattering at an orbital resonance or (ii) a nonresonant scattering caused by a molecular cloud. As a consequence, the star moves inward or outwards depending on whether angular momentum is lost or gained. They referred to the process of change of angular momentum as "churning". This churning process should preserve the star circular orbit. However, if the scattering changes the epyciclic amplitude but not the angular momentum the process is called "blurring". The chemical evolution model of Schönrich and Binney (2009) including churning and blurring described the evolution of thick and thin disks, with the stars of both disks formed in situ. The predicted radial mixing of stellar populations with high vertical velocity dispersion from inner regions of the disk to the solar vicinity, provided an explanation of why the vertical velocity dispersion increases with age with a steeper slope than predicted by theory. The abundance gradients in the ISM were also reproduced thanks to the inward radial gas flows. Finally, they also suggested that the thick disk could have been formed by migrated stars.

In principle, stellar migration can explain the observed spread in the age- $[\mathrm{Fe} / \mathrm{H}]$ and $[\mathrm{X} / \mathrm{Fe}]$ vs. $[\mathrm{Fe} / \mathrm{H}]$ relations in the solar vicinity, as first pointed out by Francois and Matteucci (1993), who considered the different stellar birthplaces of stars now observed in the solar neighbourhood, as derived by Grenon (1989). Several chemical evolution models included stellar migration (Kubryk et al. 2015; Minchev et al. 2011, 2013; Spitoni et al. 2015; Buck 2020; Vincenzo and Kobayashi 2020; Sharma et al. 2020; Khoperskov et al. 2020) and reached different conclusions. Kubryk et al. (2015) considered both blurring and churning in a detailed chemical evolution model with the thin disk forming inside-out and concluded that stellar migration of old and metal poor stars from the inner regions to the solar vicinity helps in reproducing the features of both thin and thick disk, and in particular the 
spread in the age-metallicity relation which is larger than the spread observed in the $[\alpha / \mathrm{Fe}]$ vs. $[\mathrm{Fe} / \mathrm{H}]$ relations. Francois and Matteucci (1993) suggested that the spread in the $[\alpha / \mathrm{Fe}]$ relations should be less than the spread in $[\mathrm{Fe} / \mathrm{H}]$, since their differences at various Galactocentric distances are less than the differences in the age- $[\mathrm{Fe} / \mathrm{H}]$ relations. Minchev et al. (2011, 2013) presented chemo-dynamical models including stellar migration, triggered by mergers at high redshift and central bar at later times, and suggested that a sizeable fraction of old metal-poor high $[\alpha /$ $\mathrm{Fe}$ ] stars can reach the solar vicinity from the inner regions. They also claimed to reproduce the majority of observational features in the thick and thin disks. Spitoni et al. (2015), included in a simple way stellar migration in the two-infall model, following the prescriptions of Minchev et al. (2013), and assumed velocities of 1 $\mathrm{km} / \mathrm{s}$ for the migrating stars. They were able to reproduce very well the G-dwarf metallicity distribution in the solar vicinity, accounting for those high metallicity stars that were missing in previous works (e.g., Chiappini et al. 1997). In fact, they found that a fraction not larger than $20 \%$ of the stars in the solar vicinity might have migrated from the inner regions. These results are shown in Fig. 24. As one can see, the effect of stellar migration improves the agreement with the observed G-dwarf metallicity distribution at high metallicity, but it does not have a dramatic effect on the predictions, even if all the stars observed in the solar neighbourhood were originating from somewhere else. This is an important consideration, because it tells us that stellar migration can improve the agreement with observations but is not crucial to explain them. Vincenzo and Kobayashi (2020), by means of cosmological simulations for the formation and evolution of the Milky Way, concluded that stellar migration involves old and metal rich stars and that it occurs more outwards than inwards (see Fig. 25). This means that stellar migration is not so important to interpret data in the solar neighbourhood. They also suggested that stellar migration acts in flattening the stellar metallicity gradient and this could explain the different slopes of the observed nebular and stellar gradients. Buck (2020), as mentioned before, explained the bimodality in the $[\alpha / \mathrm{Fe}]$ vs. $[\mathrm{Fe} / \mathrm{H}]$ plane for the thick and thin disk by means of stellar migration. He suggested that low- $\alpha$ stars originated from both inner and outer disk, while most of the high- $\alpha$ stars originated from the inner disk. Moreover, Sharma et al. (2020) by means of a chemo-dynamical model, suggested that the $[\alpha / \mathrm{Fe}]$ bimodality cannot be reproduced without stellar migration. On the other hand, Khoperskov et al. (2020), by means of N-body simulations, concluded that stellar migration has a negligible effect on the $[\alpha / \mathrm{Fe}]$ vs. $[\mathrm{Fe} / \mathrm{H}]$ relation. In conclusion, there is not yet agreement about the importance and the entity of stellar migration along the thin disk of the Milky Way.

\section{The evolution of light elements in halo and disks}

Light elements ( $\mathrm{H}, \mathrm{D}, \mathrm{He}$ and ${ }^{7} \mathrm{Li}$ ) have been produced during the Big Bang but some of them are produced also in stars, such as ${ }^{4} \mathrm{He},{ }^{3} \mathrm{He}$ and ${ }^{7} \mathrm{Li}$. Deuterium instead is only destroyed inside stars. The primordial abundances of light elements, and in particular that of $\mathrm{D}$, are quite important, since they are related to the baryon density of the Universe $\left(\Omega_{B}\right)$, or equivalently to the baryon-to photon ratio $(\eta)$. Data 

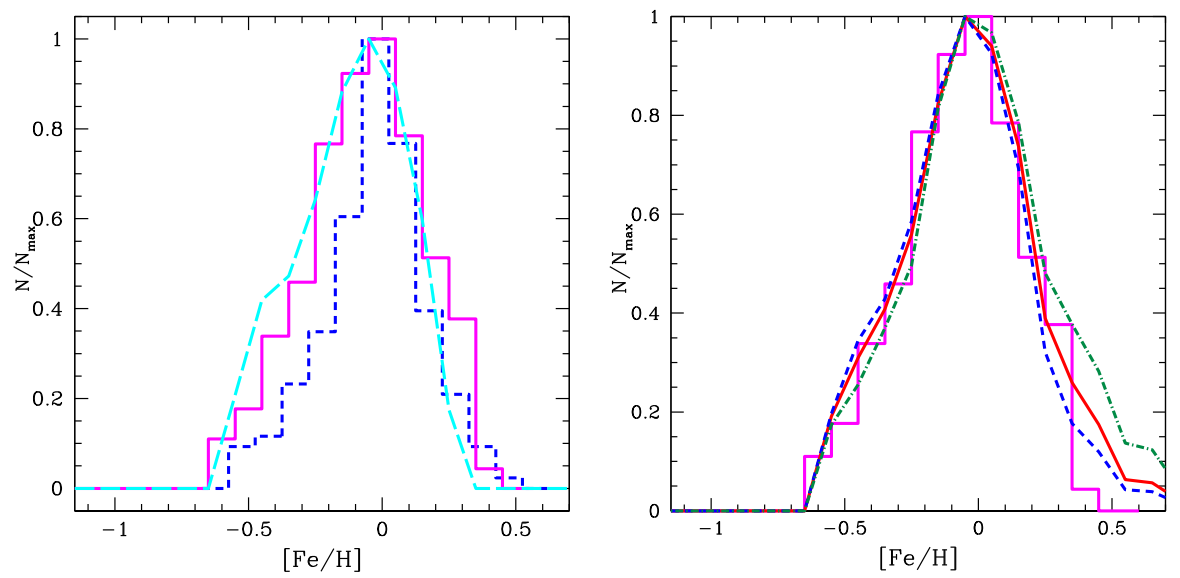

Fig. 24 Plot of the G-dwarf metallicity distribution in the solar vicinity. Left panel: comparison between data and model results without stellar migration (cyan line). The data are from Adibekyan et al. (2013) (solid magenta histogram) and Fuhrmann (2011) (short-dashed blue histogram). Right panel: comparison between data and models with stellar migration. The dashed blue line refers to the predictions of a case with migration ( $10 \%$ and $20 \%$ of the stars born at 4 and $6 \mathrm{kpc}$, respectively, which end up at $8 \mathrm{kpc}$ ). Solid red line is migration ( $20 \%$ and $40 \%$ of stars born at 4 and $6 \mathrm{kpc}$, respectively, which end up at $8 \mathrm{kpc}$ ), and the green dashed-dotted line is extreme migration (all stars born at 4 and $6 \mathrm{kpc}$ which end up at $8 \mathrm{kpc}$ ). Image reproduced with permission from Spitoni et al. (2015), copyright by AAS

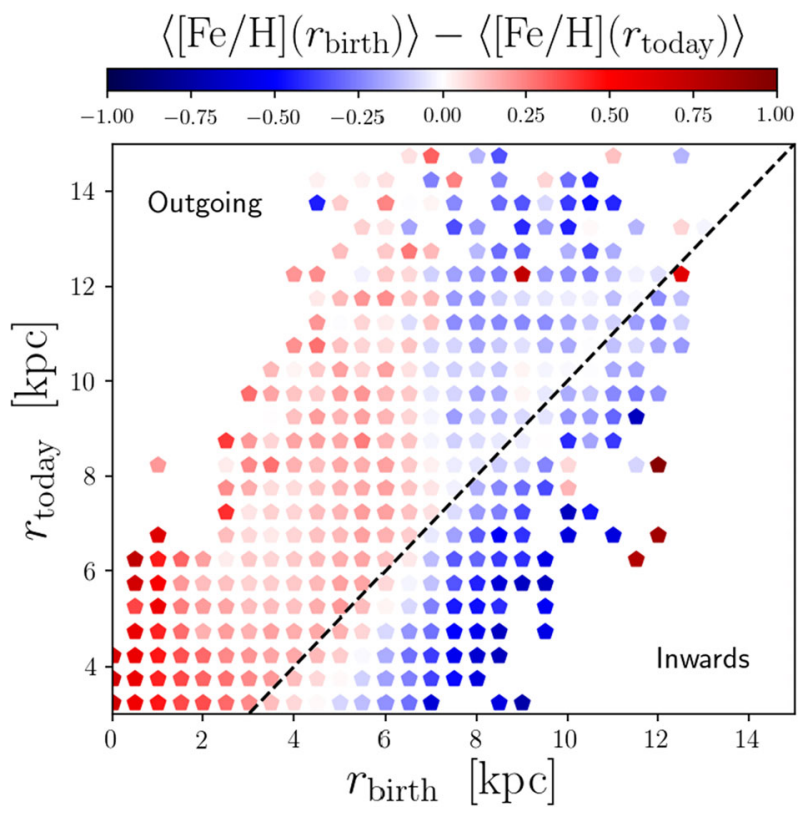

Fig. 25 Difference between the average $[\mathrm{Fe} / \mathrm{H}]$ at the birth-radius, $\left\langle[\mathrm{Fe} / \mathrm{H}]\left(r_{\text {birth }}\right)\right\rangle$, and the average $[\mathrm{Fe} /$ $\mathrm{H}]$ at the present-day radius, $\left\langle[\mathrm{Fe} / \mathrm{H}]\left(\mathrm{r}_{\text {today }}\right)\right\rangle$, in the $r_{\text {today }}-r_{\text {birth }}$ diagram for the thin-disk stars. The dashed line indicates no migration. Image reproduced with permission from Vincenzo and Kobayashi (2020), copyright by AAS 
on cosmic microwave background (CMB) can provide directly the value of $\eta$ and, therefore, of $\Omega_{B}$, and thus the primordial abundances of $\mathrm{D}, \mathrm{He}$ and Li. Results from the Wilkinson Microwave Anisotropy Probe (WMAP, Spergel et al. 2003) first, and then Planck Collaboration (Coc et al. 2014) were used to derive the primordial abundances. All the primordial abundances derived from the CMB agree with observations, except for ${ }^{7} \mathrm{Li}$. Primordial D is measured in high redshift unevolved objects, such as Damped Lyman- $\alpha$ Systems (DLAs), obtaining a lower limit to the primordial value (e.g., O’Meara et al. 2006; Pettini et al. 2008). The primordial abundance of ${ }^{4} \mathrm{He}$ is measured in unevolved galaxies, such as blue compact ones (e.g., Aver et al. 2015; Peimbert et al. 2017; Izotov et al. 2014) and intergalactic medium (see Cooke and Fumagalli 2018). Concerning ${ }^{7} \mathrm{Li}$, its primordial abundance was believed to be that shown by old metal poor halo stars $(-2.4 \leq[\mathrm{Fe} / \mathrm{H}] \leq-1.4)$, which show a rather flat Li abundance (the Spite-plateau, Spite and Spite 1982), and in particular $12+\log (\mathrm{Li} / \mathrm{H})=\mathrm{A}(\mathrm{Li})=2.05-2.2 \mathrm{dex}$ (Spite and Spite 1986; Bonifacio and Molaro 1997). On the other hand, WMAP results, confirmed later by Planck, suggested a primordial Li abundance of $\sim 2.6$ dex. Moreover, more recent work on very metal poor halo stars (Sbordone et al. 2010; Meléndez et al. 2010; Hansen et al. 2014; Bonifacio et al. 2015) challenged also the existence of the Spite plateau: in fact, for metallicities $[\mathrm{Fe} / \mathrm{H}]<-2.8 \mathrm{dex}$, the $\mathrm{Li}$ abundance was found to decrease, but Aguado et al. (2019) found a halo star with $[\mathrm{Fe} / \mathrm{H}]<-6.1$ dex and $\mathrm{Li}$ abundance close to the Spite plateau value.

\subsection{The chemical evolution of Li}

Here, we will focus on the Galactic evolution of the abundance of ${ }^{7} \mathrm{Li}$ : in fact, the abundance of ${ }^{7} \mathrm{Li}$ seems to increase from metal poor $([\mathrm{Fe} / \mathrm{H}]<-1.0 \mathrm{dex})$ to metal rich stars. This growth is seen by looking at the abundance of ${ }^{7} \mathrm{Li}$ in the stars lying on the upper envelope of the points in the diagram $\mathrm{A}(\mathrm{Li}) \mathrm{vs}$. $[\mathrm{Fe} / \mathrm{H}]$ (Rebolo et al. 1988), namely, the youngest stars and meteorites. This is due to the fact that ${ }^{7} \mathrm{Li}$ is very easily destroyed in stars during their evolution, and therefore, the ${ }^{7} \mathrm{Li}$ diagram shows a quite large spread, according to the different degree of destruction of this element in stars in different evolutionary phases. This can be clearly seen in Fig. 26, where the most recent $\mathrm{Li}$ data are compared to predictions of chemical evolution models. The increase by roughly a factor of ten in the Li abundance during the Galactic lifetime is here reproduced by assuming that ${ }^{7} \mathrm{Li}$ is produced in stars and ejected into the ISM. There is a main channel for producing ${ }^{7} \mathrm{Li}$ in stars: there should be a site in which the reaction ${ }^{3} \mathrm{He}(\alpha, \gamma){ }^{7} \mathrm{Be}$ can occur, but ${ }^{7} \mathrm{Be}$ should be rapidly transported by convection in regions of lower temperature, where it decays into ${ }^{7} \mathrm{Li}$ by $\mathrm{k}$-capture. This is known as Cameron-Fowler mechanism. Another channel proposed in the literature is the $v$-process occurring in massive stars (Woosley et al. 1990): as the core of a massive stars collapses to form a neutron star, the flux of neutrinos originating from the neutronization process is so intense that it can interact with the overlying shells of heavy elements. Neutrinos excite heavy element atoms and even He to particle unbound levels. 


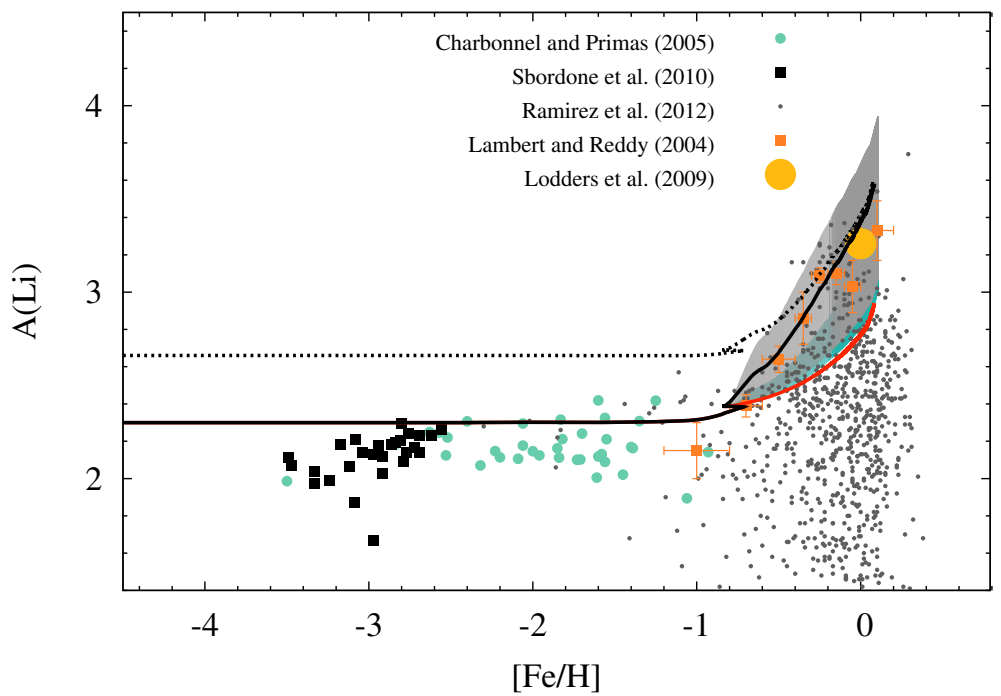

Fig. 26 Relation $\mathrm{A}(\mathrm{Li})$ vs. $[\mathrm{Fe} / \mathrm{H}]$ for the solar neighbourhood stars and meteorites, compared to the predictions of chemical evolution models (lines and coloured areas). The dotted line refers to a model starting from the primordial $\mathrm{Li}$ abundance deduced from WMAP and Planck, while the continuous line refers to a primordial abundance of $2.2 \mathrm{dex}$, and corresponding to the Spite plateau. Image reproduced with permission from Izzo et al. (2015), copyright by AAS

Several stellar ${ }^{7} \mathrm{Li}$ producers have been proposed so far and they are: CC-SNe, giant stars, AGB-stars, novae and cosmic rays. D'Antona and Matteucci (1991) tested the contribution of novae to the chemical enrichment in ${ }^{7} \mathrm{Li}$, and concluded that this contribution cannot be neglected and that is probably responsible for the steep rise of $\mathrm{A}(\mathrm{Li})$ for $[\mathrm{Fe} / \mathrm{H}]>-1.0$ dex. None of the other suggested sources could explain that because CC-SNe and AGB stars have too short lifetimes and red giant stars produce too little $\mathrm{Li}$. On the other hand, novae are systems contributing to chemical enrichment on long timescales and in principle, although many uncertainties are still present in Li nucleosynthesis in novae (see José et al. 2003; José and Hernanz 2007), they could produce the right amount of ${ }^{7} \mathrm{Li}$ (see Starrfield et al. 2020). The first and only claimed detection of ${ }^{7} \mathrm{Li}$ in novae is from Izzo et al. (2015), while detection of ${ }^{7} \mathrm{Be}$, which decades into ${ }^{7} \mathrm{Li}$, seems to be more common (Tajitsu et al. 2015, 2016; Molaro et al. 2016; Izzo et al. 2018, but see also Shore and De Gennaro Aquino 2020). Figure 26 demonstrates two predictions for Li evolution: one for the case in which the primordial $\mathrm{Li}$ is that shown by the Spiteplateau (continuous line) and the other for the case in which we assume the Planck primordial $\mathrm{Li}$ abundance (dotted line). In this latter case, we should assume that ${ }^{7} \mathrm{Li}$ has been destroyed in halo stars to the level of the Spite plateau. This difference between the value of $\mathrm{Li}$ in the Spite plateau and that derived from Big Bang nucleosynthesis based on Planck results, is called "cosmological Lithium problem" (see Matteucci et al. 2021). In both cases of primordial Li, the steep rise of its abundance in disk stars is well reproduced by the contribution of novae. The nova rate was computed as in Eq. (46) and the parameter $\delta$ constrained to reproduce an 
observed present time nova rate in the Galaxy of 20 events $\mathrm{yr}^{-1}$, in agreement with recent estimates from Della Valle and Izzo (2020).

From the analysis of the previous figures, we can see that the Li abundance in halo stars is roughly constant except perhaps for very metal poor stars, while in disk stars the Li abundance is increasing. Recently, Li data for thick and thin disk stars have become available and in thin disk stars is evident a bending of the $\mathrm{Li}$ abundance at the highest observed metallicities (Guiglion et al. 2016; Fu et al. 2018), although more recent data from Randich et al. (2020) do not show such a bending of $\mathrm{Li}$ abundance in stars of open clusters at high metallicity (up to [Fe/ $\mathrm{H}] \sim+0.3$ dex). Several explanations have been proposed for such a bending, if real, and they are: (i) stellar migration from the inner disk (Guiglion et al. 2019), (ii) Li yields depending on metallicity (Prantzos et al. 2017), (iii) a decreasing fraction of novae with increasing metallicity (Grisoni et al. 2019). In Fig. 27 presents the results of Grisoni et al. (2019) for the predicted evolution of ${ }^{7} \mathrm{Li}$ in thick and thin disk stars. In this model, the two disks evolve in parallel with different SFRs, as already mentioned in the previous paragraphs. The fraction of nova systems in the $\mathrm{IMF}$, the parameter $\delta$ in Eq. (46), is assumed to decrease with metallicity, as suggested by observations of Gao et al. (2014, 2017) and Yuan et al. (2015). In Fig. 27, we show both the results with a constant fraction of novae and with a variable one. As one can see in the left panel of this figure, the thick disk does not show any bending and the $\mathrm{Li}$ abundance presents only a little increase starting at around $[\mathrm{Fe} / \mathrm{H}] \sim-0.3$ dex.

\subsection{The chemical evolution of D}

Deuterium is only destroyed to form ${ }^{3} \mathrm{He}$ in stars, so it is very low or completely exhausted in highly evolved systems. Romano et al. (2003) presented the predictions of a chemical evolution model concerning $D$ in the solar vicinity. The amount of $D$ destroyed during Galactic evolution is called astration. The predicted
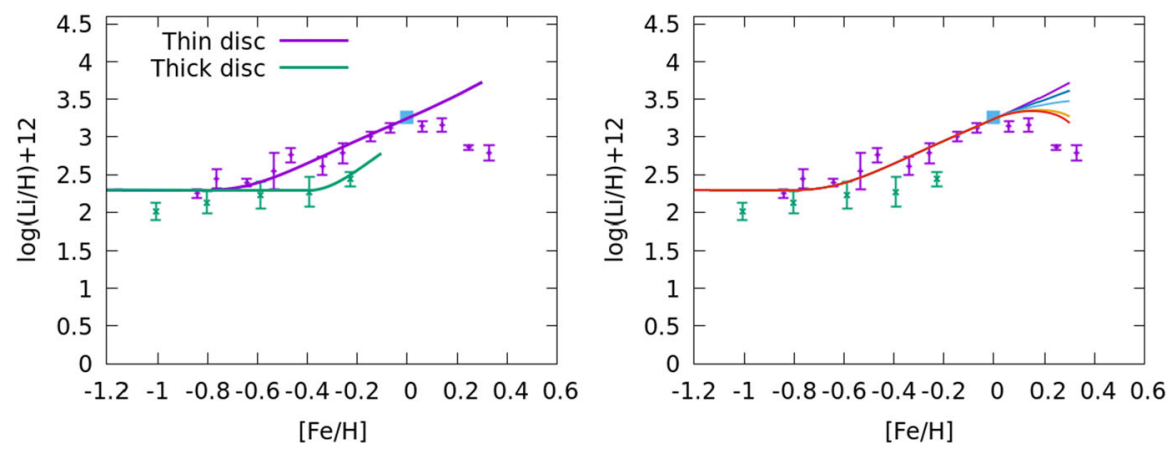

Fig. 27 Relation $\mathrm{A}(\mathrm{Li})=\log (\mathrm{Li} / \mathrm{H})+12$ vs. $[\mathrm{Fe} / \mathrm{H}]$ for the solar vicinity thick and thin disk stars. Left panel: the evolution of $\mathrm{Li}$ abundance in the thick (green line) and thin (purple line) disk. Right panel: the evolution of $\mathrm{Li}$ in the thin disk under various assumptions about the fraction of nova system decreasing with metallicity. The data are from AMBRE (Guiglion et al. 2016). Image reproduced with permission from Grisoni et al. (2019), copyright by the authors 


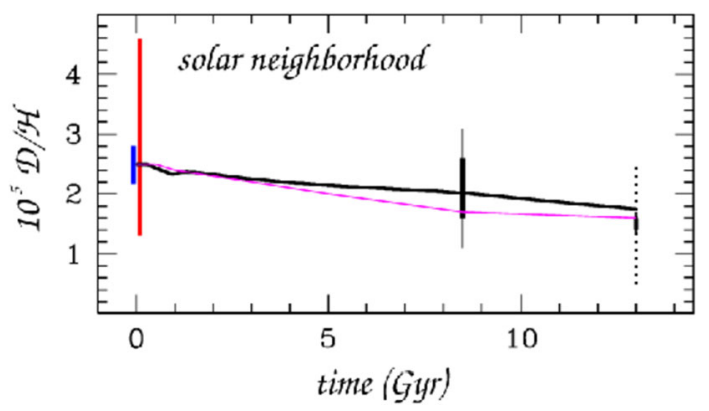

Fig. 28 Temporal evolution of the abundance of $D$ in the solar vicinity. The models are from Romano et al. (2003). The data are from WMAP (black bar) and high redshift QSO (red bar) for the primordial D and the bars represent the uncertainty in the data. The bar at the time of the formation of the Solar System reflects the measurements of Geiss and Gloeckler (1998), while the little bar at 13 Gyr represents ISM data from Linsky (1998). The other points at 13 Gyr reflect the measurements from Copernicus HSTGHRS, IMAPS, STIS, and FUSE (see Vidal-Madjar et al. 1998; Jenkins et al. 1999; Moos et al. 2002; Hoopes et al. 2003), and show a large spread. Image reproduced with permission from Romano et al. (2003), copyright by RAS

astration in the solar vicinity is quite low $\left(X_{D, p} / X_{D, t_{\mathrm{Cal}}} \sim 1.5\right)$, as shown in Fig. 28, where the primordial $D$ was assumed to be $(D / H)_{p}=2.5 \times 10^{-5}$, consistent with the abundance derived by WMAP. The first detailed chemical evolution models for $D$ demonstrating a low astration factor were presented by Steigman and Tosi (1992) and Dearborn et al. (1996). Observationally, $D$ abundances measured in QSO absorbers at high redshift provide a good lower limit to the primordial value and they agree with the WMAP estimate. In Fig. 28, the $D$ measured at the age of the formation of the Solar System ( $\sim 4.5$ Gyr ago) and in the ISM at the present time are reported. Two slightly different models are shown (see Romano et al. 2003 for details), but the astration factor of $D$ is always the same. Therefore, chemical evolution models suggest a small astration factor for $D$, at least in the solar vicinity. On the other hand, the $D$ astration in the Galactic bulge should be higher than in the solar neighbourhood, because of the higher SFR that should have occurred in the bulge, as predicted by Matteucci et al. (1999) (see later). Measurements of $D$ in the Galactic center region are from Polehampton et al. (2002), who observed the giant molecular cloud Sagittarius B2 and observed an abundance $D / H=(0.2-11) \times 10^{-6}$, from Lubowich et al. (2000) and Jacq et al. (1999) who found $D / H=(1.7 \pm 0.3) \times 10^{-6}$, a factor of ten lower than in the local ISM. Concerning the $D$ abundance in the local ISM, it should be said that there is a spread relative to measurements in different lines of sight. The interpretation of such a spread is not clear and it can be related to the different degree of dust condensation for $D$ in different spatial regions.

\subsection{The chemical evolution of ${ }^{3} \mathrm{He}$ and ${ }^{4} \mathrm{He}$}

The abundance of ${ }^{3} \mathrm{He}$ is strictly related to that of $\mathrm{D}:{ }^{3} \mathrm{He}$ is produced during $\mathrm{H}$-burning in low and intermediate mass stars, but is also destroyed to produce 
heavier elements. The stellar production of ${ }^{3} \mathrm{He}$ is still uncertain, since it depends on the treatment of extra-mixing and subsequent Cool Bottom Process in low mass stars (Charbonnel and Lagarde 2010). From a theoretical point of view we remind a few seminal papers on the evolution of ${ }^{3} \mathrm{He}$ by Galli et al. (1995), Dearborn et al. (1996) and Lagarde et al. (2012). From an observational point of view, it is not easy to measure the ${ }^{3} \mathrm{He}$ abundance: Balser et al. $(1997,1999)$ measured its abundance in Galactic planetary nebulae (PNe), and Bania et al. (2002) in Galactic HII regions. Data and model results show a light increase of the ${ }^{3} \mathrm{He}$ abundance during Galactic lifetime.

In addition, ${ }^{4} \mathrm{He}$ is produced and destroyed in stars of all masses and its abundance increases slightly in time. Starting from a primordial value of $Y_{P}=$ $\sim 0.24$, its abundance reaches $\sim 0.28$ in the Sun and it can reach 0.35 in the Galactic bulge (Renzini 1994), although there is no real consensus about this high value.

\section{The chemical evolution of the Galactic bulge}

In this section, we will describe what we have learned up to now about the chemical evolution of the Galactic bulge and Galactic center region. In the last years, several spectroscopic and photometric surveys have been devoted to the understanding of the formation and evolution of the bulge: in particular, Gaia-ESO (Gilmore et al. 2012), APOGEE (Majewski et al. 2017), Argos (Freeman et al. 2013), GIBS (Zoccali et al. 2014), VVVX (Minniti et al. 2010), (Perryman et al. 2001), BRAVA (Rich et al. 2007; Kunder et al. 2012) and COMBS (Lucey et al. 2019). From these surveys we can obtain information on chemical abundances, kinematics and ages of bulge stars.

Wyse and Gilmore (1992) summarized different scenarios proposed for the bulge formation, they are: (a) accretion of extant stellar systems which settled in the center of the Galaxy by dynamical friction, with negligible star formation in situ; (b) the bulge formed from gas accumulated at the center of the Galaxy, either by mergers or simply reflecting initial conditions, evolving independently of the other Galactic components. In this case, the star formation was either rapid or slow; (c) the bulge formed from gas supply from inflow of metal-rich gas from the thick disk or halo. In addition, in this case, the formation process could have been rapid or slow. For a recent review on the chemodynamical history of the Galactic bulge, we address the reader to Barbuy et al. (2018). The main conclusions of this review can be summarized as: (i) the bulk of bulge stars are old and span a metallicity range $-1.5 \leq[\mathrm{Fe} / \mathrm{H}] \leq+0.5$. The analysis of the stellar populations suggest that the overall bulge formed on a timescale $<2 \mathrm{Gyr}$ and that is barred and follows cylindrical rotation, and the more metal rich stars trace a Box/Peanut structure.

\subsection{The evolution of $\alpha$-elements in the bulge}

From a chemical evolution point of view, the best tool to derive the timescale of bulge formation is represented by the abundances and abundance ratios in bulge 
stars. In particular, the stellar MDF and the plots $[\mathrm{X} / \mathrm{Fe}]$ vs. $[\mathrm{Fe} / \mathrm{H}]$ can impose important constraints on the history of star formation in the bulge. Matteucci and Brocato (1990) were the first trying to reproduce the MDF obtained by Rich (1988), and concluded that one should assume a fast bulge formation with intense SFR and IMF flatter than in the solar vicinity. Under these conditions, they predicted that the bulge stars should have shown a longer plateau in the $[\alpha / \mathrm{Fe}]$ vs. $[\mathrm{Fe} / \mathrm{H}]$ plot than in the solar neighbourhood, as shown in Fig. 29. In other words, the knee in such a plot, due to the intervention of Type Ia SNe in producing Fe (time-delay model), should occur at a higher metallicity than for stars in the solar vicinity. The reason for that relies in the fact that a high SFR enriches the ISM very fast in Fe by means of CC-SNe, so that when the SNe Ia start occurring the Fe abundance in the ISM is already high and due only to the contribution of CC-SNe. The contrary occurs in systems with low SFR, such as irregular and dwarf spheroidal galaxies (see Matteucci 2012). Such a prediction for the Galactic bulge was confirmed later by other theoretical papers (e.g., Ballero et al. 2007; Cescutti and Matteucci 2011; Grieco et al. 2012b), suggesting a fast formation for the majority of bulge stars $(0.3-0.5$ Gyr). Since this is a precise prediction of the time-delay model coupled with the star formation history, if observed, it represents a confirmation of the timedelay model itself. A different position of the knee in the $[\alpha / \mathrm{Fe}]$ ratio in the bulge stars has been observed both in Gaia-ESO (Rojas-Arriagada et al. 2017) and APOGEE (Zasowski et al. 2019) surveys. In both cases, the knee in the $[\mathrm{Mg} / \mathrm{Fe}]$ ratio is found at $[\mathrm{Fe} / \mathrm{H}]_{\mathrm{knee}}=-0.37 \pm 0.09 \mathrm{dex}$, which is $\sim 0.06 \mathrm{dex}$ higher than the knee found in the thick disk in the solar vicinity. Although this difference is not remarkable, this is an indication that the bulge formed faster than the solar neighbourhood. However, this knee refers to the so-called metal poor bulge, because

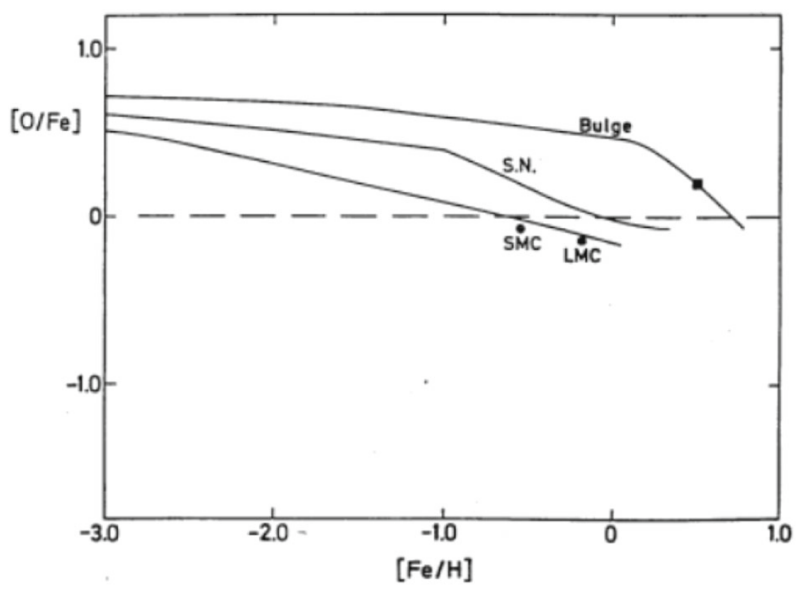

Fig. 29 Predicted behaviour of the $[\mathrm{O} / \mathrm{Fe}]$ ratio vs. $[\mathrm{Fe} / \mathrm{H}]$ in three different galaxy types: the solar vicinity, the Galactic bulge and the Magellanic Clouds (LMC and SMC). The difference between the chemical models describing these galaxies is the SFR. The SFR is highest for the bulge and lowest for LMC and SMC. Reported in the figure are also some observational points relative to the bulge and SMC and LMC, available at that time. Image reproduced with permission from Matteucci and Brocato (1990), copyright by AAS 

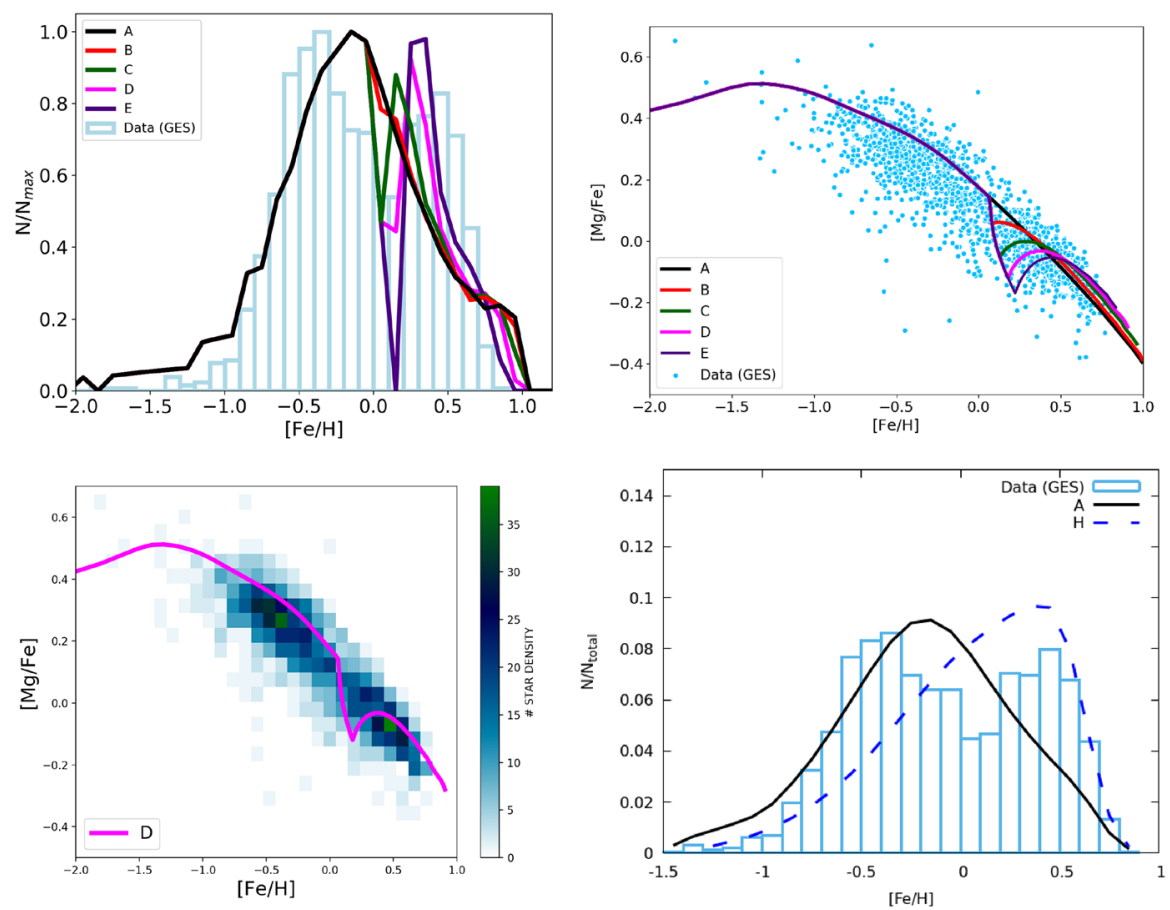

Fig. 30 Upper panel left: Predicted MDF in the Galactic bulge for models with continuous star formation with stops of the duration of 50,150, 250 and $350 \mathrm{Myr}$, compared to Gaia-ESO data. As one can see, longer is the stop in star formation and deeper is the dip between the two populations. The model which best reproduces the data is the one with a stop of $250 \mathrm{Myr}$. Upper panel right: predicted $[\mathrm{Mg} / \mathrm{Fe}] \mathrm{vs}$. $[\mathrm{Fe} /$ $\mathrm{H}]$ in the Galactic bulge, for the same models, also compared to Gaia-ESO data. Lower panel left: a density plot for the Gaia-ESO data compared to the results of the model with 250 Myr stop in star formation. Lower panel right: predicted MDF in the case in which the second peak is due to accreted inner disk stars. The data are the same as in the upper left panel. Image reproduced with permission from Matteucci et al. (2019), copyright by the authors

recently, two main bulge stellar populations have been found, one metal poor and one metal rich. In fact, a bimodal metallicity distribution was observed by Hill et al. (2011) and then confirmed by more recent papers (e.g., Rojas-Arriagada et al. 2017; Schultheis et al. 2017; Zoccali et al. 2017). On the other hand, Bensby et al. $(2011,2013,2017)$ found a multi-modal distribution with the last population formed 3 Gyr ago (see also Ness and Freeman 2016). Several studies of the ages of bulge stars (Clarkson et al. 2011; Johnson et al. 2011; Valenti et al. 2013; Renzini et al. 2018) suggested that the majority of them is old (age $>10 \mathrm{Gyr}$ ). Bernard et al. (2018) inferred the history of star formation in the bulge and concluded that only $10 \%$ of bulge stars are younger than $5 \mathrm{Gyr}$, but this fraction raises to $20-25 \%$ in the metal rich peak. Chemical models should be able to reproduce the MDF as well as the $[\alpha / \mathrm{Fe}]$ vs. $[\mathrm{Fe} / \mathrm{H}]$ plot. In Fig. 30, we show the predicted and observed MDF of bulge stars from Gaia-ESO data and a good agreement is reached for a model assuming a stop of $250 \mathrm{Myr}$ duration in the SFR. In addition, in this figure, the plot of $[\mathrm{Mg} / \mathrm{Fe}]$ vs. $[\mathrm{Fe} / \mathrm{H}]$ for the same model is shown: here, the discontinuity due to 
the stop in star formation is not so evident as in the MDF, but is probably present, as shown in the left lower panel of Fig. 30, where a density plot for $[\mathrm{Mg} / \mathrm{Fe}]$ is reported and a clear lower stellar density is visible in correspondance to the dip in the predicted $[\mathrm{Mg} / \mathrm{Fe}]$ ratio. An alternative explanation for the two peaks in the MDF is that the more metal rich and younger population could have been accreted from the inner disk. Such a population should be younger than the one of the first peak, with lower average $[\alpha / \mathrm{Fe}]$ ratios and more metal rich. As we have already pointed out, from a kinematical point of view the metal rich population is associated with the Boxy/Peanut X-shaped bulge (Zoccali et al. 2017), while the more metal poor population seems to be isotropically distributed. This kinematical characteristics is consistent with either the accreted inner disk stars or with the stop in the star formation (see Debattista et al. 2017). Matteucci et al. (2019) found that a good agreement with data is reached if the metal poor population formed very fast, on a timescale lower than $0.5 \mathrm{Gyr}$ and with an IMF containing more massive stars (see also Johnson et al. 2014) than in the solar vicinity, thus confirming all the previous results. Moreover, they found that the fraction of stars younger than 5 Gyr should be not higher than $10 \%$, in agreement with the observational estimate of Bernard et al. (2018), but at variance with other suggestions (e.g., Bensby et al. 2017; Haywood et al. 2016). In conclusion, chemical evolution models cannot help in understanding the dynamical evolution of the bulge but they can impose important constraints on the formation timescales of the bulge stellar populations. The suggestion of a very fast formation for the metal poor spheroidal bulge favors a formation in situ. On the other hand, the metal rich population could have been accreted from the thin or thick disk but we cannot exclude that it could have also formed "in situ".

\subsection{The evolution of $n$-capture elements in the bulge}

The $n$-capture elements, in particular r-and s-process elements, are characterized by a large spread in their abundances in solar vicinity stars, especially in halo stars. This spread can be explained by assuming inhomogeneous mixing, as discussed in Sect. 6.1.2. Abundances of $\mathrm{Eu}, \mathrm{Ba}, \mathrm{La}$ and $\mathrm{Zr}$ have been measured also in bulge stars, as shown in Fig. 31, where we show the $[\mathrm{X} / \mathrm{Fe}]$ vs. $[\mathrm{Fe} / \mathrm{H}]$ plots for such elements. As one can see, concerning $\mathrm{Eu}$, a pure r-process element, the [Eu/Fe] follows the behaviour of $\alpha$-elements, being overabundant relative to the Sun at low metallicity and decreasing for higher metallicities, as it appears to be the average trend in solar vicinity stars. As we have already discussed, this fact suggests a fast Eu production at early times which can be obtained either if the Eu producers are MNS or CC-SNe or both. In the case of MNS, this is achieved either by assuming that all MNS have very short coalescence times, or that the coalescence timescales are distributed in a large range of values, but the fraction of MNS is higher at early times (Simonetti et al. 2019). The only difference between bulge and halo data is the smaller spread observed in the former. For what concerns $\mathrm{Ba}, \mathrm{Sr}, \mathrm{La}$ and $\mathrm{Zr}$, they are mainly produced by low mass stars as s-process elements but also with a contribution from r-process in massive stars, although recently it has been suggested that they can originate also from s-process occurring in fastly rotating massive stars (Chiappini et al. 2011). In Fig. 31, we can see that Ba, La and $\mathrm{Zr}$ in bulge stars tend 


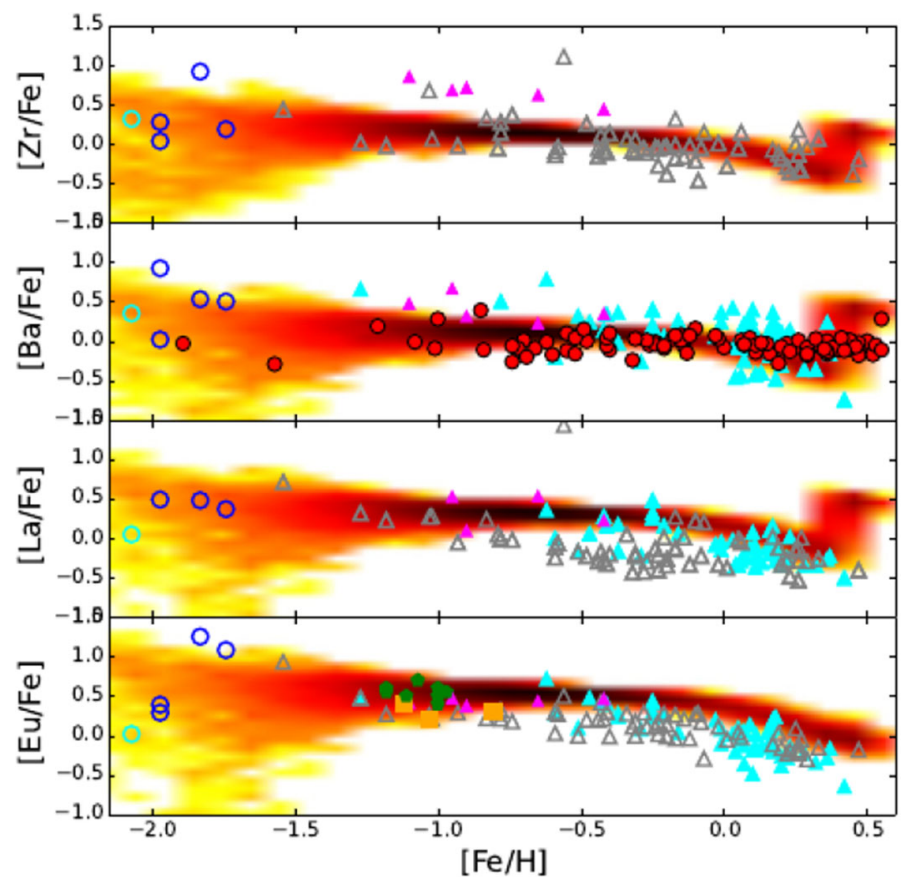

Fig. 31 Observed and predicted $[\mathrm{Zr}, \mathrm{Ba}, \mathrm{La}, \mathrm{Eu} / \mathrm{Fe}]$ vs. $[\mathrm{Fe} / \mathrm{H}]$ relations. The data are a compilation from Barbuy et al. (2018) and the predictions of the inhomogeneous model of Cescutti et al. (2018) are described by the orange-yellow shaded area. Image reproduced with permission from the arXiv preprint of Barbuy et al. (2018)

to decrease with increasing metallicity. This is different to what happens in the solar vicinity (see Fig. 9), where $[\mathrm{Ba} / \mathrm{Fe}]$ increases up to $[\mathrm{Fe} / \mathrm{H}] \sim-2.0 \mathrm{dex}$ and then decreases slightly for higher metallicity. Cescutti et al. (2018) applied the inhomogeneous model to the bulge and the results are also shown in Fig. 31. For the evolution of the abundances of $\mathrm{Zr}, \mathrm{La}, \mathrm{Nd}$ and $\mathrm{Eu}$ in the bulge see also Johnson et al. (2012).

\subsection{Chemical evolution of the Galactic centre}

In Grieco et al. (2015) and Ryde et al. (2016), the evolution of the central region of the Galaxy ( $\sim 500$ pc in radius) was studied by comparing chemical models to spectroscopic stellar data and estimates of the SFR. The adopted chemical model for the Galactic centre is very similar to that of the entire bulge for what concerns the $\mathrm{SF}$ and infall rates. The main conclusion was that the Galactic central region should have evolved as the rest of the bulge, with intense SFR (a suggested SFR efficiency of $\sim 25 \mathrm{Gyr}^{-1}$ ) and, therefore, on a short timescale, confirming all the previous chemical works. The spectroscopic stellar data for stars in this very central region were derived from very high resolution data taken in three different fields. These studies have suggested that the stars in the central region lie in a large range of 
metallicities $(-1.2<[\mathrm{Fe} / \mathrm{H}]<+0.3)$. They also found a lack of a $[\alpha / \mathrm{Fe}]$ gradient, similarly to what has been found in the rest of the bulge. This result again points towards the conclusion that the whole bulge formed very quickly (see also Rich et al. 2012). Figure 32 shows the models for the Galactic centre region compared to the data.

\section{The chemical evolution of the Galactic halo}

The Galactic halo is formed by field stars and GCs and it contains only a small fraction of the total stellar mass of the Milky Way. Hartwick (1976) derived the MDF of 60 Galactic GCs, and showed that is quite different from the metallicity distribution of the G-dwarfs in the solar neighbourhood. He compared the data with the predictions of the Simple Model (see Sect. 3) and concluded that this model predicts too few very metal poor stars. He solved this problem in two ways: (i) by lowering the yield per stellar generation by a factor of 13 , or (ii) by gas loss, with the gas going later to form the disk. However, the first assumption resulted in a model with a very large halo mass/disk mass ratio, thus leaving only the second assumption as feasible. A recent estimate of the halo stellar mass is from Mackereth and Bovy (2020) and is $M_{* \text { halo }}=1.3_{-0.2}^{+0.3} \cdot 10^{9} M_{\odot}$.

Prantzos (2003) also attempted to model the halo chemical evolution and concluded that an outflow model can well reproduce the peak and the shape of the halo MDF, but it fails in describing the lowest metallicity region, especially if I.R.A. is relaxed. He suggested, as a solution, an early gas infall phase for the halo. Such a conclusion was shared lately by Brusadin et al. (2013), who suggested a halo model

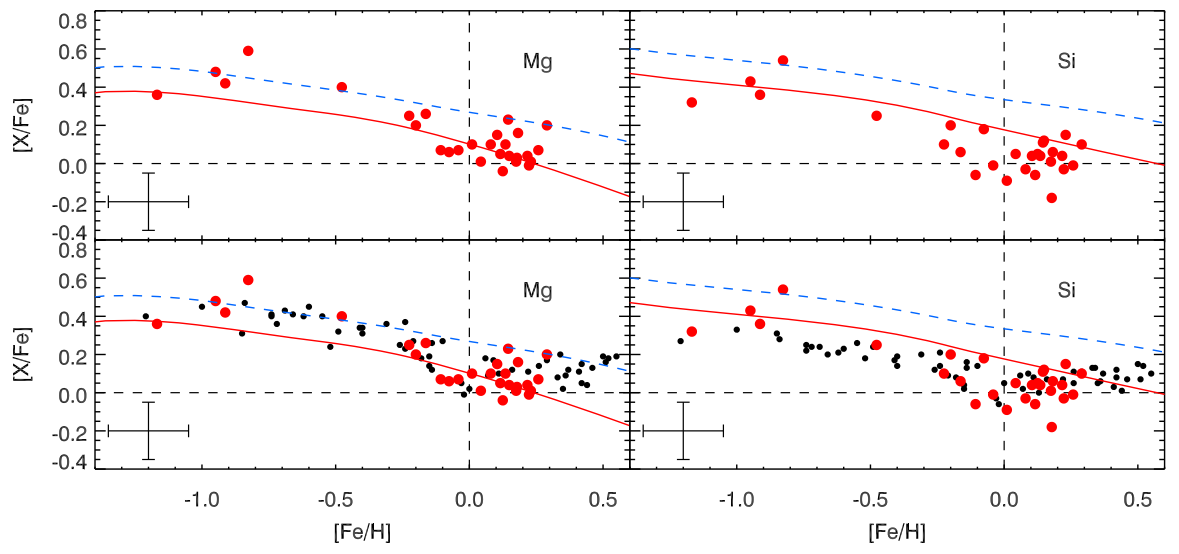

Fig. 32 Predicted and observed $[\mathrm{Mg} / \mathrm{Fe}]$ and $[\mathrm{Si} / \mathrm{Fe}]$ vs. $[\mathrm{Fe} / \mathrm{H}]$ for the inner $500 \mathrm{pc}$ of the Galactic bulge. The abundance ratios for $28 \mathrm{M}$ giants derived by Ryde et al. (2016) are shown with red dots. The predictions are represented by the two curves: the dotted curve represents a model adopting standard yields for the $\mathrm{Mg}$ and $\mathrm{Si}$ production, whereas the continuous line is the prediction of the same model except for the yields of $\mathrm{Mg}$ and $\mathrm{Si}$ which have been decreased by a factor of 1.35 (Grieco et al. 2015). In the lower panels are also reported the abundances based on micro-lensed dwarfs in the 'outer' bulge by Bensby et al. (2013), marked with black smalldots. Image reproduced with permission from Ryde et al. (2016), copyright by AAS 
including infall and outflow at the same time. All of these models assumed that all halo stars formed "in situ". In Fig. 33, we report the results of Brusadin et al. (2013) in the framework of the two-infall model, where the halo forms by infall but without outflow and in the case where both infall and outflow are present.

A recurrent question about the Galactic stellar halo, and not yet completely understood, is: Has the Galactic halo formed in situ or some or all stars have been accreted from dwarf satellite galaxies?. May be the answer is that part of the halo stars formed in situ and part have been accreted (Carollo et al. 2007, 2012). In fact, it is likely that in the past there have been many tidal interactions between the Milky Way and its satellites and a large fraction of these satellites are seen today as stellar streams or tidal debris (e.g., Helmi et al. 1999; Simion et al. 2019). The best diagnostic for understanding the origin of the stars in the halo is to look at their chemical abundance ratios and compare them with those observed in dwarf galaxies, which are expected to show particular abundance patterns. For example, dwarf spheroidal (dSphs) and ultra faint dwarf galaxies (UfDs) around the Milky Way show $[\alpha / \mathrm{Fe}]$ ratios at low metallicity lower than in the Galactic halo stars (see Matteucci 2012).

It is worth noting that Nissen and Schuster (2010) showed that the metal rich tail of the Galactic halo is made of two distinct stellar populations. They suggested that the population with a lower $[\alpha / \mathrm{Fe}]$ should have been accreted from dwarf galaxies. In Fig. 34, we show a comparison between $[\alpha / \mathrm{Fe}]$ ratios in UfDs satellites of the Milky Way and Galactic halo stars, together with models for UfDs. It is easy to recognize that the $[\alpha / \mathrm{Fe}]$ ratios overlap at very low metallicity but many halo stars have ratios larger than those in UfD stars. Spitoni et al. (2016), compared the abundance patterns $([\alpha / \mathrm{Fe}],[\mathrm{Ba} / \mathrm{Fe}]$ vs. $[\mathrm{Fe} / \mathrm{H}])$ of dSphs with those of Galactic halo stars and suggested that the majority of halo stars should have formed in situ.
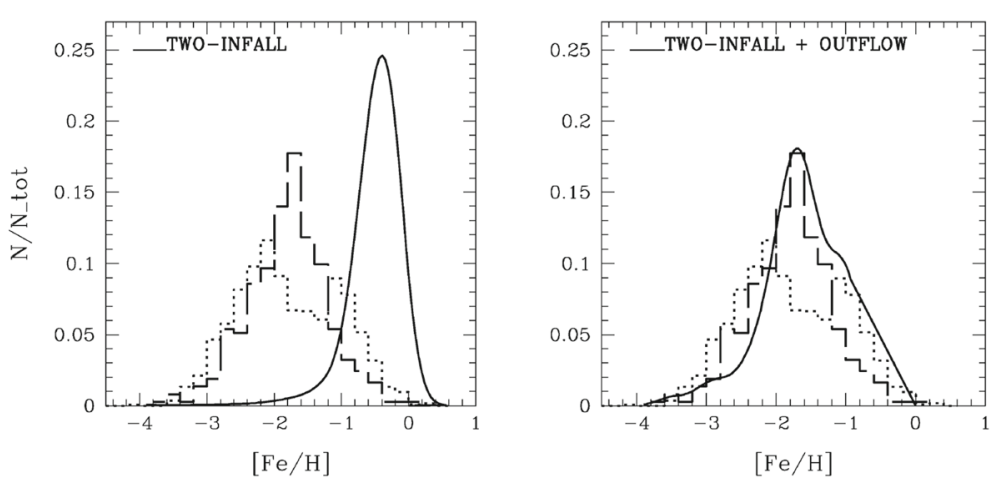

Fig. 33 Stellar halo metallicity distribution function in the framework of the two-infall model (Chiappini et al. 1997), where no outflow is considered during halo formation (left panel, solid curve) and in the twoinfall plus outflow framework (right panel, solid curve). The data are represented by the histograms, where the dashed one represents data from Ryan and Norris (1991) and the dotted one the data from Schörck et al. (2009). The theoretical predictions have been smoothed by a Gaussian function with a variance equal to the data error of 0.2 dex. Image reproduced with permission from Brusadin et al. (2013), copyright by ESO 

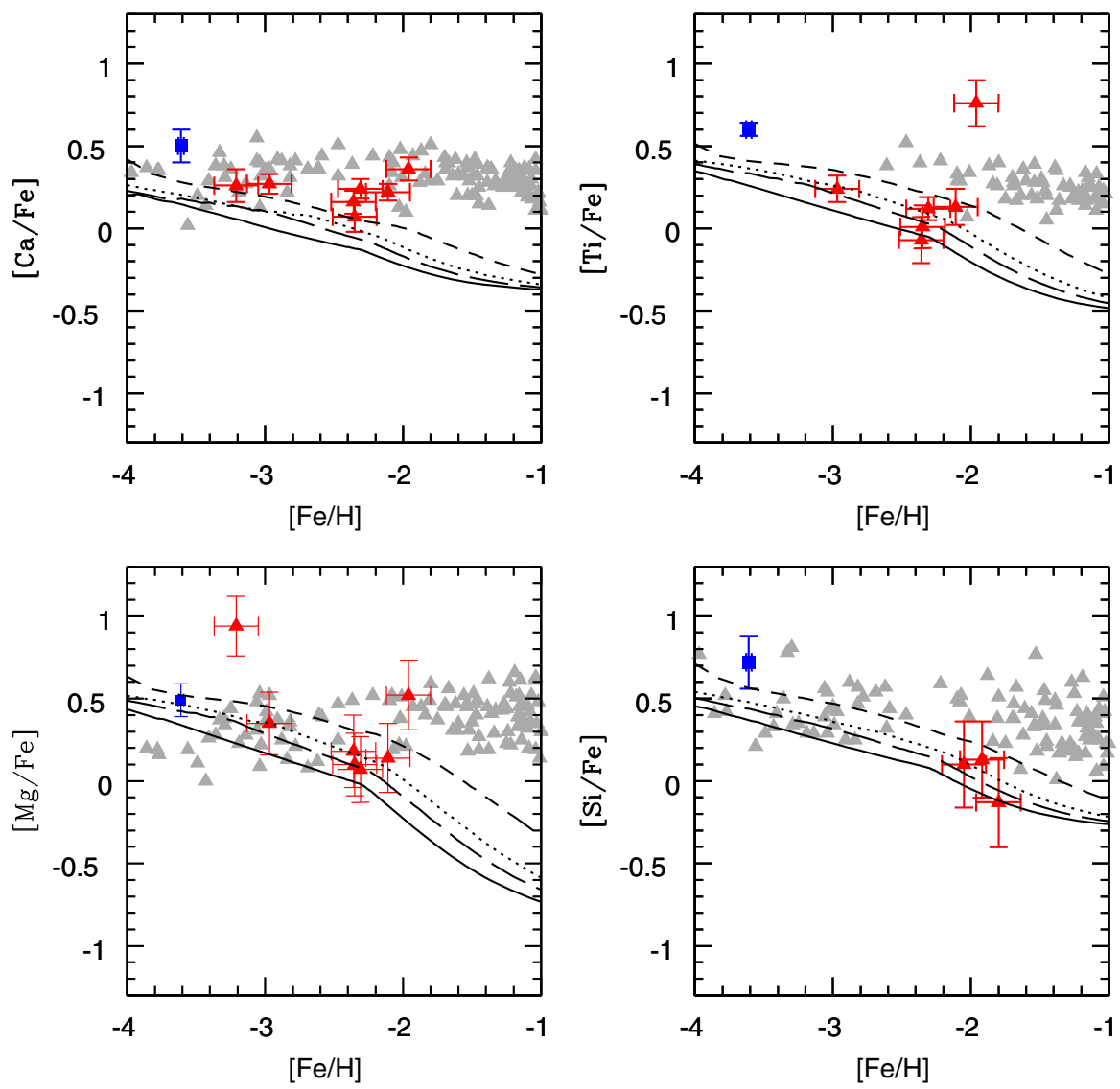

Fig. 34 Comparison between $[\alpha / \mathrm{Fe}]$ ratios in the stars of Boötes I (red and blue points) and halo stars (grey triangles). Overimposed are the results of models for Boötes I from Vincenzo et al. (2014). The blue square is from Norris et al. (2010) and the red triangles from Gilmore et al. (2013). The data for halo stars are from Gratton et al. (2003), Reddy et al. (2003), Reddy et al. (2006) and Cayrel et al. (2004). Image reproduced with permission from Vincenzo et al. (2014), copyright by the authors

Recently, it has been revealed a metal-rich component in the inner Galaxy halo, showing a peculiar elongated shape (Belokurov et al. 2018) like a "sausage". This object represents the record of a head-on major collision that the Galaxy should have experienced more than $10 \mathrm{Gyr}$ ago with a dwarf galaxy rather massive. The progenitor, now disrupted, of this "sausage" is called Gaia-Enceladus or Gaia Sausage. A sample of stars in the "sausage" were selected by Helmi et al. (2018), and show a $[\alpha / \mathrm{Fe}]$ vs. $[\mathrm{Fe} / \mathrm{H}]$ pattern similar to those of dSphs, namely, they show lower $[\alpha / \mathrm{Fe}]$ ratios than metal poor halo stars of the same metallicity. Vincenzo et al. (2019) modeled the chemical evolution of Gaia-Enceladus, assuming that this object evolved as a dwarf spheroidal galaxy with lower SFR than in the Galaxy. By fitting the $[\alpha / \mathrm{Fe}]$ vs. $[\mathrm{Fe} / \mathrm{H}]$ relation, as well as the MDF of Gaia Sausage, they predicted a median age for its stars of $12.33_{-1.36}^{+0.92} \mathrm{Gyr}$ and a total stellar mass at the 
time of merging ( $\sim 10 \mathrm{Gyr}$ ago $)$ of $M_{* \text { Sausage }}=5 \times 10^{9} M_{\odot}$. They also suggested that the merging event might have contributed to inhibit the gas accretion onto the Galaxy, thus producing a gap in the SFR, in agreement with predictions from chemical evolution models (e.g., Chiappini et al. 1997; Spitoni et al. 2019). In the context of the two-infall model, it is likely that Gaia Sausage was cannibalised by the Galaxy at the end of the first infall episode.

Mackereth and Bovy (2020) studied the Galactic halo in the space of $[\mathrm{Fe} / \mathrm{H}]$, $[\mathrm{Mg} / \mathrm{Fe}]$ and $e$ (orbital eccentricity) by means of red giant counts from APOGEE DR14, and concluded that the majority of the total stellar halo mass was accreted, and that $\sim 30-50 \%$ of this accreted mass belonged to Gaia Enceladus, whose mass was then estimated to be $M_{* \text { Sausage }}=3 \pm 1 \times 10^{8} M_{\odot}$, lower than previous estimates. On the other hand, Iorio and Belokurov (2021) presented results about a large sample of RRLyrae detected by Gaia. Their chemo-kinematics analysis suggested that the inner halo (inside $10 \mathrm{Kpc}$ ) likely contains RRLyrae formed in situ, while other RRLyrae out to $30 \mathrm{kpc}$ are consistent with disk kinematics and are young and metal rich. In our opinion, a good criterion to establish if the halo stars were accreted is represented by low $[\alpha / \mathrm{Fe}]$ ratios at low $[\mathrm{Fe} / \mathrm{H}]$ (see Fig. 34), and even better by $[\mathrm{Ba} / \mathrm{Fe}]$ at low $[\mathrm{Fe} / \mathrm{H}]$, as suggested by Spitoni et al. (2016).

Finally, concerning the possibility of inhomogeneous chemical evolution of the Galactic halo, we address the reader at Fig. 19, where we already discussed the spread observed in the abundances of neutron-process elements and how it can be reproduced by assuming inhomogeneous chemical evolution.

\section{Chemo-dynamical models in cosmological context}

Up to now, we have mostly discussed how to model the chemical evolution of the Milky Way by means of the so-called "analytical chemical models", although only a small fraction of them is really analytical, being the largest fraction constituted by numerical models. These models, that we will call pure chemical models, both analytical and numerical, although possessing a nice predictive power, do not assume any cosmological paradigm for galaxy formation. The need for a cosmological framework for galaxy formation has led to the adoption of more complex chemo-dynamical models for the formation and evolution of galaxies. As we will see, in the majority of cases the conclusions of the latter models confirm what found by pure chemical models but adding information on the stellar and gas kinematics. The cosmological galaxy formation models belong to two categories: (i) semi-analytical models and (ii) numerical simulations. The cosmological framework is always the $1 \mathrm{CDM}$ one (a cold dark matter Universe with a cosmological constant), which assumes a hierarchical formation of galaxies, a scenario, where the largest galaxies form by accretion of smaller ones.

The basic ingredients of these models are:

- the cosmological model; 
- the dark matter haloes: in particular, it should be given the abundance of haloes of different mass, the formation history of each halo (the merger tree) and the internal structure of each halo, in terms of radial density and angular momentum.

Then, other more complicated ingredients which are related to the physics of the gas, and they are far more uncertain than the treatment of gravitational instability, should be added. These processes are: star formation, gas cooling, stellar feedback, chemical evolution, gas and stellar dynamics, galaxy mergers. These models followed the successful study of the evolution of dark matter in the $1 \mathrm{CDM}$ scenario, with the aim of understanding also the evolution of the baryonic component, which presents a higher complexity due to the many poorly understood physical processes involved. Among the first semi-analytical approaches to galaxy formation and evolution are those of White and Frenk (1991), Kauffmann et al. (1993) and Cole et al. (1994); they combine the growth of dark matter haloes with simple parametrizations of the physics of baryons. The other common approach, which is complementary to the semi-analytical one, is to adopt hydrodynamical simulations, and models of this type have been constructed either for isolated or populations of galaxies (e.g., Katz 1992; Mihos and Hernquist 1994; Navarro and White 1994; Steinmetz and Mueller 1994; Cen and Ostriker 1999; Springel and Hernquist 2003; Kawata and Gibson 2003a, b; Nakasato and Nomoto 2003). It should be said that initially, given the complexity of these models, the chemical evolution has often been treated in a simplistic way, by adopting for example I.R.A., that allows us to follow only the chemical enrichment from CC-SNe, and/or adopting the closed-box model assumption. Thomas (1999) and Thomas and Kauffmann (1999) were among the first who included enrichment by Type Ia SNe in semi-analytical models but in the framework of the closed-box model (no infall or outflow). In the following years, detailed chemical evolution was included in hydrodynamical simulations (e.g., Kobayashi 2004; Kobayashi et al. 2007; Tornatore et al. 2007; Jiménez et al. 2015) and in semi-analytical models studying the properties of elliptical galaxies (e.g., Nagashima et al. 2005; Arrigoni et al. 2010; Calura and Menci 2011). In most of these papers, stellar lifetimes and detailed chemical enrichment from Type Ia SNe were considered, following the basic approach of Greggio and Renzini (1983), Matteucci and Greggio (1986) and Chiappini et al. (1997).

\subsection{The Milky Way in a cosmological context}

Here, we are interested in modelling the Milky Way galaxy, and therefore, we will present only models whose results can be compared to our Galaxy. In the last few years, several papers dealing with chemical evolution of a Milky Way like galaxy in a cosmological context appeared in the literature (Colavitti et al. 2008; Kobayashi and Nakasato 2011; Brook et al. 2012; Few et al. 2014; Loebman et al. 2016; Grand et al. 2018; Mackereth et al. 2018; Haynes and Kobayashi 2019; Vincenzo and Kobayashi 2020; Clarke et al. 2019; Buck 2020; Vincenzo and Kobayashi 2020), but we will discuss only some of the results obtained by means of the above cosmological simulations. 


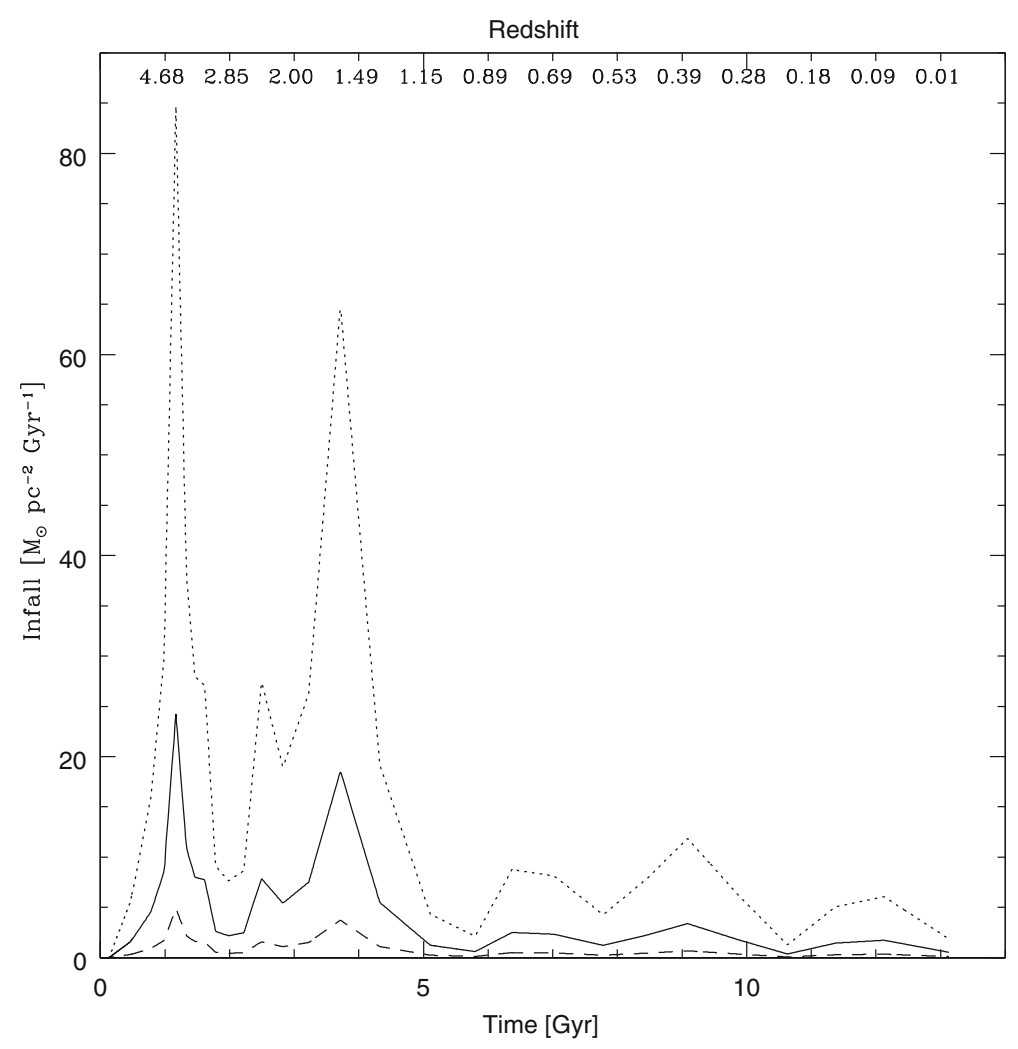

Fig. 35 Cosmologically motivated gas infall law for the formation of the Milky Way. The continuous line refers to a Galactocentric distance of $8 \mathrm{kpc}$ (solar neighbourhood), the dotted line to $4 \mathrm{kpc}$ and the dashed line to $14 \mathrm{kpc}$. The redshift is also indicated in the figure. Image reproduced with permission from Colavitti et al. (2008), copyright by ESO

Colavitti et al. (2008) aimed at finding a cosmologically motivated gas infall law for the formation of the Milky Way, since in pure chemical models such a law is assumed a priori as an exponential or gaussian function. They assumed that baryons assemble like dark matter and selected an infall law compatible with the formation of a disk galaxy like the Milky Way. The selection occurred by means of cosmological N-body simulations adopting the public code GADGET2 (Springel 2005). Not without surprise, they concluded that the best infall law is very similar to that of the two-infall model (see Eq. 50) of Chiappini et al. (1997). In Fig. 35, we show their infall laws at different Galactocentric distances: as one can see, the law presents two peaks occurring at $\sim 1$ and $\sim 4$ Gyr and this clearly resembles to the two major infall episodes predicted by the two-infall model. Clearly, the abundance patterns for the solar vicinity, as predicted by this cosmological infall law, are very similar to the results of the two-infall model, including the gap in star formation between the two peaks. It should be noted that in this formulation no timescale for gas accretion are given a priori, as it is instead done in pure chemical models. 
Kobayashi and Nakasato (2011) performed detailed chemo-dynamical simulations of a Milky-Way like galaxy, and starting from cold dark matter conditions they included supernova (CC-SNe and Type Ia SNe) feedback as well as chemical enrichment, and followed the evolution of the gas abundances of several elements from $\mathrm{O}$ to $\mathrm{Zn}$. In this kind of model, the star formation history goes like this: the CDM initial fluctuations grow into nodes and filaments and small collapsed haloes of gas and dark matter are created. Inside the haloes the gas cools radiatively and star formation takes place starting from redshift $z \sim 15$. According to the hierarchical formation of dark haloes, subgalaxies merge to form larger galaxies and the merging induces star formation. In this picture, the Galactic bulge forms during the initial starburst which is triggered by the assembly of gas-rich subgalaxies with masses in the range $\sim(5-10) \times 10^{9} M_{\odot}$ at redshift $z \geq 3$. Due to its angular momentum, the gas then accretes onto the plane to form a disk which grows inside-out, as suggested by the chemical evolution models of the previous paragraphs (e.g., Larson 1976; Matteucci and Francois 1989; Chiappini et al. 2001). In this disk, the star formation has a longer timescale than the bulge, thanks to the self-regulation of supernova feedback. Many satellites are successively accreted and disrupted, but there is no major merger event after $z \sim 2$, so the disk structure is retained. The assumed SN Ia progenitor model is the single degenerate one, as presented in Kobayashi and Nomoto (2009). They compared the chemical and kinematical results for bulge, disk and halo stars with observations: the $[\mathrm{X} / \mathrm{Fe}]$ vs. $[\mathrm{Fe} / \mathrm{H}]$ plots were predicted for several chemical species in the solar neighbourhood and in the bulge, as well as the MDFs for the thin disk at the solar ring, the thick disk and the halo. In Fig. 36, we report the predicted [X/Fe] vs. $[\mathrm{Fe} / \mathrm{H}]$ relations for the solar neighbourhood at $z=0$. This plot shows the effect of the time-delay model discussed before, since the SN progenitors, stellar lifetimes and stellar yields are the same as in the pure chemical evolution models. Therefore, the physical interpretation of the behaviours of the $[\mathrm{X} / \mathrm{Fe}]$ vs. $[\mathrm{Fe} / \mathrm{H}]$ diagrams is always the same, and is due to the different contributions to chemical enrichment by $\mathrm{SNe}$ with different stellar lifetimes. What is different from pure chemical evolution models, is that the evolution of the ISM is not homogeneous and some spread in the abundance ratios is naturally predicted, as evident in Fig. 36.

Several cosmological simulations of the Milky Way in the last few years, were aimed at explaining the apparent bimodal distribution of the $[\alpha / \mathrm{Fe}]$ ratios in disk stars, as already discussed previously (see Fig. 14). Mackereth et al. (2018), by means of EAGLE simulation, analysed this distribution for 133 Milky Way-like galaxies. They found that the bimodality occurring in the Milky Way is rare, since it appears only in $\sim 5 \%$ of their simulated galaxies. They suggested this as the consequence of an early gas accretion episode occurring at an atypically-rapid growth. Their interpretation of the bimodal sequence is related to two different infall episodes, similar to the two-infall model of Chiappini et al. (1997) and Spitoni et al. (2019). Clearly, the first infall episode has to be faster to ensure rapid star formation avoiding substantial pollution from Type Ia SNe, and thus creating stars with high $[\alpha / \mathrm{Fe}]$ ratios. However, it is worth noting that the Type Ia SN rate adopted in Mackereth et al. (2018) is quite different from those described in this review, 

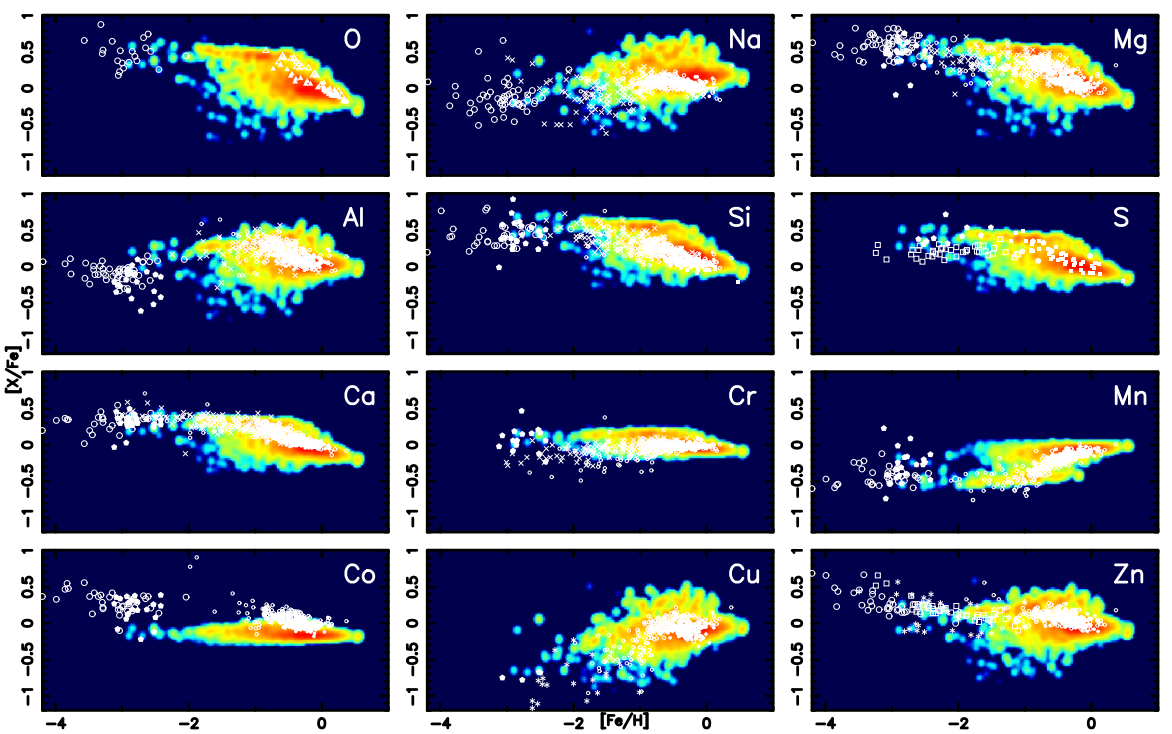

Fig. 36 Predicted and observed $[\mathrm{X} / \mathrm{Fe}]$ vs. $[\mathrm{Fe} / \mathrm{H}]$ relations in the solar neighbourhood. The $[\mathrm{Fe} / \mathrm{H}] \mathrm{range}$ includes halo, thick and thin disk stars. The data are indicated with white dots, large open circles, filled pentagons, crosses, small filled and small open circles, filled triangles, open triangles, filled squares, filled pentagons, open squares and asterisks. The contours show the predicted frequency distribution of stars in the simulated Milky Way-like galaxy, and red represents the highest frequency. It is worth noting that here a bimodality is predicted for all the studied elements. See data references in Kobayashi and Nakasato (2011). Image reproduced with permission from Kobayashi and Nakasato (2011), copyright by AAS

because the majority of SNe Ia occurr almost instantaneously, thus loosing the effect of the time-delay model. Other differences are present between the two approaches (cosmological and pure chemical evolution), for example the cosmological model predicts a continuous increase of metallicity over time rather than tending to an equilibrium value (see also Grand et al. 2018). However, a common interpretation of the $[\alpha / \mathrm{Fe}]$ bimodality among pure chemical models and cosmological ones is related to the time-delay model for chemical enrichment, as well as the two distinct infall episodes and a hiatus in the star formation between them. We remind here that Grisoni et al. (2017) suggested also another interpretation of the bimodality, as due to two parallel and distinct episodes of gas infall, not separated by a stop in the star formation. Another common feature to all models of the Milky Way, both cosmological and purely chemical, is the inside-out formation of the thin disk. Vincenzo and Kobayashi (2020) also reproduced the bimodality in the $[\alpha / \mathrm{Fe}]$ ratios, as shown by the APOGEE-DR16 data, as well as the stellar age distribution from APOKASC-2. They also confirmed the inside-out formation of the thin disk and studied stellar migration, as already discussed (see Fig. 25). Their chemodynamical simulations is based on Gadget-2 (Springel 2005) but include many relevant processes, such as radiative cooling depending on metallicity and $[\alpha / \mathrm{Fe}]$ ratios, star formation, thermal feedback from stellar winds and $\mathrm{SNe}$ and detailed 
nucleosynthesis (elements up to Zn) from Type Ia SNe and CC-SNe. Haynes and Kobayashi (2019) treated separately the evolution of elements derived from neutron-capture elements. In Fig. 37, we show some results from Vincenzo and Kobayashi (2020), where the bimodality in $[\alpha / \mathrm{Fe}]$ ratios is reproduced together with the stellar ages; the bimodality in their model is attributed to both infall and ouflow events that they find during the evolution of the Milky Way. Finally, as already mentioned, other cosmological simulations (e.g., Buck 2020; Sharma et al. 2020), as well as chemical models (Anders et al. 2017) have attributed the bimodality to stellar migration.

In conclusion, models of the Milky Way in a cosmological context have in general confirmed the suggestions of pure chemical models and in particular the timescales for the formation of the various Galactic components, the time-delay model, the interpretation of $[\alpha / \mathrm{Fe}]$ bimodality in disk stars and the inside-out formation of the thin disk.
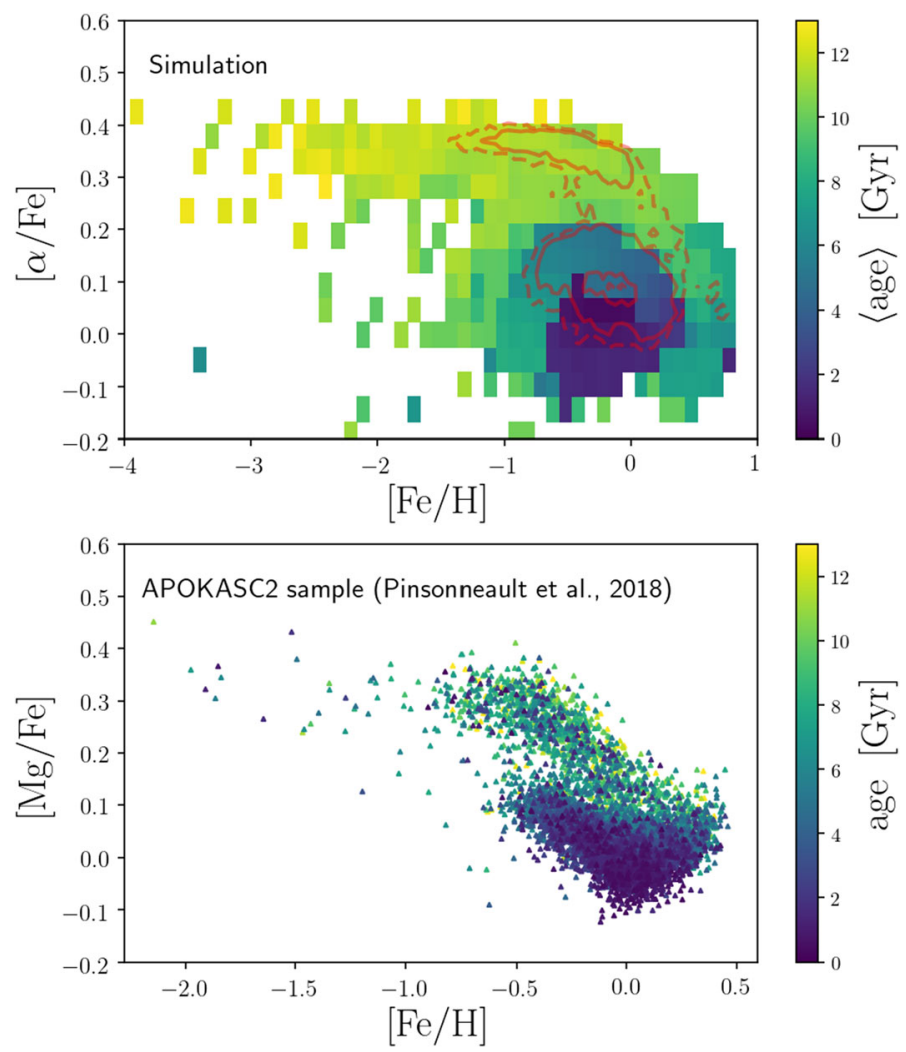

Fig. 37 Top panel: predicted $[\alpha / \mathrm{Fe}]$ vs. $[\mathrm{Fe} / \mathrm{H}]$ together with the age distribution in the simulated galaxy at the present time. Bottom panel: the observed age-distribution of the stars from the second APOKASC catalog (Pinsonneault et al. 2018) in the $[\alpha / \mathrm{Fe}]$ vs. $[\mathrm{Fe} / \mathrm{H}]$ diagram. Image reproduced with permission from Vincenzo and Kobayashi (2020), copyright by the authors 


\section{Conclusions and discussion}

In this review, we have described in detail the chemical modeling of the Milky Way, divided in its main components: stellar halo, thick and thin disks and bulge. Particular attention has been devoted to the evolution of single chemical elements from $\mathrm{H}$ to heavy elements, and on how to impose constraints on the formation and evolution of our Galaxy by comparison theory-observations. The detailed chemical evolution of the Milky Way has been studied in the past years mainly by means of pure chemical models, either analytical or numerical. Moreover, in recent times, several attempts to model the Milky Way in a cosmological context have appeared, by means of either semi-analytical models or hydrodynamical numerical simulations. We have shown the predictive power of all of these models as well as indicated the many uncertainties still present in modeling the Milky Way. In the following, we will summarize the most important still open questions and how the results achieved by models have contributed to a better understanding of the formation and evolution of the Milky Way.

The most important questions are:

- Did the halo stars form in situ or were they accreted?

This is not yet clear, although it seems that at least a fraction of halo stars have been accreted, since they show lower $[\alpha / \mathrm{Fe}]$ ratios. In fact, the best tool to ascertain this point is represented by the abundance ratios vs. metallicity relations. A recent important discovery has been the realization that $10 \mathrm{Gyr}$ ago a massive dwarf galaxy, called Gaia-Enceladus or Gaia-Sausage, has fallen into the potential well of our Galaxy. The stars of this object might represent a large fraction of the stars accreted by the Galactic halo.

- How should we explain the large spread observed in some abundance ratios in halo stars?

The abundances and abundance ratios of neutron-process elements show a particularly large spread observed in halo stars relative to other elements, such as $\alpha$ - and Fe-peak elements. The first explanation for the spread is to assume inhomogeneous evolution of the halo, but then the spread should be visible in all the elements. A tentative explanation was given by Cescutti (2008), who suggested that the different extent of the spread in the plot abundance ratio vs. metallicity is due to the different nucleosynthesis and stellar progenitors of different elements, coupled with inhomogeneous mixing.

- How did the two disks form? Thick disk formed fast, whereas the thin formed slowly? Did the thin disk form inside-out?

The two Galactic disks could have formed in a sequential way but with a halt in the star formation between the two, and by means of different gas infall events (e.g., Chiappini et al. 1997). Alternatively, the two disks could have formed in parallel, out of two independent infall events but occurring at the same time and at different rates (e.g., Grisoni et al. 2017). In both scenarios, the rate of chemical evolution must have been different in the two disks, with the thick disk evolving faster than the thin disk. Most of chemical models as well as chemodynamical cosmological simulations agree that the thin disk should have formed 
on a longer time scale than the thick disk and the halo. Late time major or minor mergers for the formation of the thin disk seem to be excluded, since they would have cancelled the abundance gradients. On the other hand, abundance gradients are favored by an inside-out formation of the thin disk, although other processes, such as inward gas flows and decreasing efficiency of star formation with the Galactocentric distance should also be present (e.g., Palla et al. 2020; Spitoni and Matteucci 2011).

- What is the cause of bimodality in the $[\alpha / \mathrm{Fe}]$ ratios in the thick and thin disks, if real?

The cause of bimodality, if confirmed to be real, is certainly a consequence of the mechanisms and timescales for the formation of the two Galactic disks. The bimodality shows that thick disk stars have larger $[\alpha / \mathrm{Fe}]$ ratios than the thin disk stars and part of them lie in the same $[\mathrm{Fe} / \mathrm{H}]$ range of thin disk stars, so that these abundance ratios appear in two sequences. The sequences look parallel in GaiaESO and AMBRE data, whereas in APOGEE data the low $[\alpha / \mathrm{Fe}]$ sequence appears rather as a plateau. This bimodal effect is indeed interesting, and it seems to be a common characteristic up to large Galactocentric distances (Queiroz et al. 2020). In any case, many have been the explanations suggested for the bimodality. Several authors suggested that a framework like that of the two-infall model can explain the bimodality, since there is a stop in the star formation between the formation of the two disks with consequent dilution and decrease of the Fe abundance. This effect had been found first by Chiappini et al. (1997), who showed that a gap in star formation of less than 1 Gyr was expected because of the second infall coupled with a threshold gas density for the star formation. To explain APOGEE data, Spitoni et al. (2019) proposed a longer gap of $\sim 4.3 \mathrm{Gyr}$, while Grisoni et al. (2017) proposed a parallel disk formation to explain the data of AMBRE survey. Other authors (e.g., Anders et al. 2017; Kubryk et al. 2015; Buck 2020; Sharma et al. 2020) have suggested that the bimodality is due to stellar migration. In addition, a late infall event occurring in the thin disk has been suggested (e.g., Calura and Menci 2009), as well as the possibility that Gaia Enceladus can have influenced the evolution of the thick disk (Grand et al. 2020).

- How important is stellar migration?

Stellar migration seems indeed to exist and most of the studies suggest that it should occur mainly from the inner to the outer Galactic thin disk regions. However, there is not a general agreement on how really important is stellar migration. It has been invoked to solve several problems including the observed spread in the abundance patterns observed in the solar neighbourhood, the $[\alpha / \mathrm{Fe}]$ bimodality as well as the existence of the thick disk itself. On the other hand, models without stellar migration can still reproduce the majority of the observed features in the solar vicinity, except for the presence of stars with super solar metallicity, for which a 10-20\% of migrated stars could be enough (see Spitoni et al. 2015). Anyway, the exact amount of solar vicinity stars which have migrated from other regions is still difficult to establish.

- How did the bulge form? How many different stellar populations are in the bulge? 
Most of the chemical studies, including chemo-dynamical cosmological simulations, relative to the Galactic bulge have suggested that it formed quickly, as a consequence of a strong burst of star formation lasting less than 1 Gyr. With high star formation efficiency, short infall timescale and an IMF with more massive stars than in the solar neighbourhood, it is possible to reproduce the $\mathrm{MDF}$ and the $[\mathrm{X} / \mathrm{Fe}]$ vs. $[\mathrm{Fe} / \mathrm{H}]$ relations for a large part of bulge stars, as first shown by Matteucci and Brocato (1990). However, there is a fraction of bulge stars which are more metal rich and associated to the Boxy/Peanut X-shaped bulge (Zoccali et al. 2017), and might have been accreted from the inner disk. The true bulge stars seem to be old and the fraction of stars younger than 5 Gyr to be no more than $10 \%$ (e.g., Bernard et al. 2018, although other studies have suggested a larger fraction of young stars, such as Bensby et al. 2017).

- How did abundance gradients along the thin disk formed? Which is the role of radial gas flows?

Abundance gradients are present along the thin disk: they have been derived from young stars, PNe and HII regions. The abundance gradients generally indicate the present time abundances along the disk, except perhaps for data of PNe which can refer also to older objects. Chemical evolution models predict abundance gradients if there is a gradient in the SFR along the disk. This can be obtained in several ways: (i) by assuming an inside-out formation of the disk by means of gas infall, with the infall timescale increasing with Galactocentric distance (e.g., Matteucci and Francois 1989; Chiappini et al. 2001, (ii) with an efficiency of star formation decreasing with Galactocentric distance (e.g., Prantzos and Boissier 2000; Colavitti et al. 2008), (iii) by assuming a gas threshold for star formation (Chiappini et al. 2001), (iv) by varying the IMF with Galactocentric distance, although this variation should imply a smaller number of massive stars at larger Galactocentric distances, at variance with the Jeans (1902) criterion for star formation; (v) by assuming radial gas flows (e.g., Lacey and Fall 1985; Portinari and Chiosi 2000; Schönrich and Binney 2009; Spitoni and Matteucci 2011). All of these processes can be at work at the same time, but while we could avoid (ii), (iii) and (iv), we cannot exclude (i) and (v). In fact, radial gas flows and inside-out formation seem to be unavoidable physical processes: in particular, the inward radial gas flows have a strong effect on the formation of abundance gradients and they are the natural consequence of gas infall. The inside-out process derives from a faster accretion in the inner denser disk regions relative to the less dense outer ones (see Larson 1976). In addition, most of the chemodynamical cosmological simulation found that the disk of a Milky Way-like spiral forms inside-out.

Another still open question is whether the abundance gradients have steepened or flattened in time, depending mostly on the assumptions on the SFR. For example, by assuming a constant star formation efficiency leads to a steepening of the gradients in time, while a variable efficiency as a function of Galactic radius induces a flattening of gradients with time. This problem 
will be solved only when we will have more data on gradients shown by old stars.

- Which stars are the main contributors of r-process elements? Merging neutron stars or supernovae or both?

The r-process elements, such as Eu, are mainly formed in massive stars either by means of explosive nucleosynthesis during the CC-SN events and/or by merging neutron stars. The second channel seems to be favored, as shown by the heavy elements which arose from the merging neutron star event associated to the detection of GW170817 (Abbott et al. 2017). Chemical evolution studies have explored the possibility that Eu can be produced only by merging events of compact objects (neutron stars and black holes) as well as by CC-SNe or both. In fact, CC-SNe alone seem to be not able to produce the right quantity of Eu to reproduce its solar abundance. The plot of [Eu/Fe] vs. $[\mathrm{Fe} / \mathrm{H}]$ clearly shows that Eu behaves like an $\alpha$-element, namely, it shows a plateau of $[\mathrm{Eu} / \mathrm{Fe}]$ at low metallicity followed by a decrease of this ratio at higher metallicities. This behaviour is interpreted by means of the time-delay model, in which elements formed on short timescales (by massive stars), such as $\alpha$-elements, at low metallicity show an overabundance relative to $\mathrm{Fe}$, which is mainly formed and ejected by Type Ia SNe on longer timescales. Therefore, from a chemical point of view, the mergers of compact objects can be the main source of Eu, if the merging timescale is very short. Matteucci et al. (2014) suggested that all systems should merge in a time of $1 \mathrm{Myr}$, otherwise one should assume a distribution of merging times together with $\mathrm{CC}-\mathrm{SNe}$ also contributing to Eu. This conclusion was shared by papers in the following years (e.g., Cescutti et al. 2015; Côté et al. 2018; Wehmeyer et al. 2019; Kobayashi et al. 2020a), unless one assumes that the fraction of merging systems should have been higher at early times (e.g., Simonetti et al. 2019; Beniamini and Piran 2019).

- Why the origin of some elements is not yet understood?

When we compare the predicted $[\mathrm{X} / \mathrm{Fe}]$ vs. $[\mathrm{Fe} / \mathrm{H}]$ relations with observations in the solar vicinity and bulge, we find that for some elements there is no agreement and these elements mainly belong to the Fe-peak group. Some of them do not behave like Fe, as one would expect on the basis of the time-delay model, and this fact suggests that we have not yet understood the nucleosynthesis of such elements, which are ${ }^{51} \mathrm{~V},{ }^{52} \mathrm{Cr},{ }^{55} \mathrm{Mn},{ }^{59} \mathrm{Co}$ and ${ }^{64} \mathrm{Zn}$. Other elements, which do not belong to the Fe-peak element group, and whose nucleosynthesis is not yet well understood are ${ }^{41} \mathrm{~K}$ and ${ }^{48} \mathrm{Ti}$. Generally, in chemical evolution models, the yields of the above mentioned species need to be changed (increased or decreased) relative to standard nucleosynthesis calculations, to reproduce their abundance patterns (e.g., François et al. 2004; Matteucci et al. 2020). Future improvements in stellar and nucleosynthesis models are expected to shed light on the origin of these elements.

Acknowledgements I would like to thank several of my past and present collaborators, whose work represents an important part of this review. They are: F. Calura, G. Cescutti, C. Chiappini, V. Grisoni, M. Molero, M. Palla, S. Recchi, D. Romano, P. Simonetti, E. Spitoni and F. Vincenzo. I am very grateful to 
the referees, Monica Tosi and Chiaki Kobayashi, carefully reading the paper and giving me many useful suggestions.

Funding Open access funding provided by Università degli Studi di Trieste within the CRUI-CARE Agreement.

\section{Declarations}

Conflict of interest The author is Editor-in-Chief of the journal The Astronomy and Astrophysics Review.

Open Access This article is licensed under a Creative Commons Attribution 4.0 International License, which permits use, sharing, adaptation, distribution and reproduction in any medium or format, as long as you give appropriate credit to the original author(s) and the source, provide a link to the Creative Commons licence, and indicate if changes were made. The images or other third party material in this article are included in the article's Creative Commons licence, unless indicated otherwise in a credit line to the material. If material is not included in the article's Creative Commons licence and your intended use is not permitted by statutory regulation or exceeds the permitted use, you will need to obtain permission directly from the copyright holder. To view a copy of this licence, visit http:// creativecommons.org/licenses/by/4.0/.

\section{References}

Abbott BP, Abbott R, Abbott TD et al (2017) GW170817: observation of gravitational waves from a binary neutron Star Inspiral. Phys Rev Lett 119(16):161101. https://doi.org/10.1103/PhysRevLett. 119.161101. arXiv:1710.05832 [gr-qc]

Adibekyan VZ, Sousa SG, Santos NC et al (2012) Chemical abundances of 1111 FGK stars from the HARPS GTO planet search program. Galactic stellar populations and planets. A\&A 545:A32. https://doi.org/10.1051/0004-6361/201219401. arXiv:1207.2388 [astro-ph.EP]

Adibekyan VZ, Figueira P, Santos NC et al (2013) Kinematics and chemical properties of the Galactic stellar populations. The HARPS FGK dwarfs sample. A\&A 554:A44. https://doi.org/10.1051/00046361/201321520. arXiv:1304.2561 [astro-ph.GA]

Aguado DS, González Hernández JI, Allende Prieto C, Rebolo R (2019) Back to the Lithium Plateau with the $[\mathrm{Fe} / \mathrm{H}]<-6$ Star J0023+0307. ApJ 874(2):L21. https://doi.org/10.3847/2041-8213/ab1076. arXiv:1904.04892 [astro-ph.SR]

Alibés A, Labay J, Canal R (2001) Galactic chemical abundance evolution in the solar neighborhood up to the iron peak. A\&A 370:1103-1121. https://doi.org/10.1051/0004-6361:20010296. arXiv:astro$\mathrm{ph} / 0012505$

Anders F, Chiappini C, Santiago BX et al (2014) Chemodynamics of the Milky Way. I. The first year of APOGEE data. A\&A 564:A115. https://doi.org/10.1051/0004-6361/201323038. arXiv:1311.4549 [astro-ph.GA]

Anders F, Chiappini C, Minchev I et al (2017) Red giants observed by CoRoT and APOGEE: the evolution of the Milky Way's radial metallicity gradient. A\&A 600:A70. https://doi.org/10.1051/ 0004-6361/201629363. arXiv:1608.04951 [astro-ph.GA]

Arcones A, Janka HT, Scheck L (2007) Nucleosynthesis-relevant conditions in neutrino-driven supernova outflows. I. Spherically symmetric hydrodynamic simulations. A\&A 467(3):1227-1248. https://doi. org/10.1051/0004-6361:20066983. arXiv:astro-ph/0612582

Argast D, Samland M, Gerhard OE, Thielemann FK (2000) Metal-poor halo stars as tracers of ISM mixing processes during halo formation. A\&A 356:873-887. arXiv:astro-ph/9911178

Argast D, Samland M, Thielemann FK, Qian YZ (2004) Neutron star mergers versus core-collapse supernovae as dominant r-process sites in the early Galaxy. A\&A 416:997-1011. https://doi.org/10. 1051/0004-6361:20034265. arXiv:astro-ph/0309237

Arrigoni M, Trager SC, Somerville RS, Gibson BK (2010) Galactic chemical evolution in hierarchical formation models-I. Early-type galaxies in the local Universe. MNRAS 402(1):173-190. https:// doi.org/10.1111/j.1365-2966.2009.15924.x. arXiv:0905.4189 [astro-ph.CO] 
Aver E, Olive KA, Skillman ED (2015) The effects of He I $\lambda 10830$ on helium abundance determinations. J Cosmol Astropart Phys 7:011. https://doi.org/10.1088/1475-7516/2015/07/011. arXiv:1503.08146 [astro-ph.CO]

Ballero SK, Matteucci F, Origlia L, Rich RM (2007) Formation and evolution of the Galactic bulge: constraints from stellar abundances. A\&A 467(1):123-136. https://doi.org/10.1051/0004-6361: 20066596. arXiv:astro-ph/0702137

Balser DS, Bania TM, Rood RT, Wilson TL (1997) The ${ }^{3} \mathrm{He}$ abundance in planetary nebulae. ApJ 483(1):320-334. https://doi.org/10.1086/304248

Balser DS, Bania TM, Rood RT, Wilson TL (1999) ${ }^{3} \mathrm{He}$ in the milky way interstellar medium: abundance determinations. ApJ 510(2):759-783. https://doi.org/10.1086/306598

Bania TM, Rood RT, Balser DS (2002) The cosmological density of baryons from observations of ${ }^{3} \mathrm{He}^{+}$ in the Milky Way. Nature 415(6867):54-57. https://doi.org/10.1038/415054a

Baraffe I, El Eid MF, Prantzos N (1992) The s-process in massive stars of variable composition. A\&A 258(2):357-367

Barbuy B (1988) Oxygen in 20 halo giants. A\&A 191:121-127

Barbuy B, Erdelyi-Mendes M (1989) Oxygen in old and thick disk stars. A\&A 214:239-248

Barbuy B, Chiappini C, Gerhard O (2018) Chemodynamical history of the galactic bulge. ARA\&A 56:223-276. https://doi.org/10.1146/annurev-astro-081817-051826. arXiv:1805.01142 [astroph.GA]

Baron E (1992) Progenitor masses of type Ib/c supernovae. MNRAS 255:267-268. https://doi.org/10. $1093 / \mathrm{mnras} / 255.2 .267$

Bekki K, Tsujimoto T (2011) Origin of chemical and dynamical properties of the galactic thick disk. ApJ 738(1):4. https://doi.org/10.1088/0004-637X/738/1/4. arXiv:1105.5864 [astro-ph.GA]

Belokurov V, Erkal D, Evans NW, Koposov SE, Deason AJ (2018) Co-formation of the disc and the stellar halo. MNRAS 478(1):611-619. https://doi.org/10.1093/mnras/sty982. arXiv:1802.03414 [astro-ph.GA]

Beniamini P, Piran T (2019) The gravitational waves merger time distribution of binary neutron star systems. MNRAS 487(4):4847-4854. https://doi.org/10.1093/mnras/stz1589. arXiv:1903.11614 [astro-ph.HE]

Bensby T, Adén D, Meléndez J et al (2011) Chemical evolution of the Galactic bulge as traced by microlensed dwarf and subgiant stars. IV. Two bulge populations. A\&A 533:A134. https://doi.org/ 10.1051/0004-6361/201117059. arXiv:1107.5606 [astro-ph.GA]

Bensby T, Adén D, Meléndez J et al (2013) Chemical evolution of the Galactic bulge as traced by microlensed dwarf and subgiant stars. V. Evidence for a wide age distribution and complex MDF. A\&A 549:A147. https://doi.org/10.1051/0004-6361/201220678. arXiv:1211.6848 [astro-ph.GA]

Bensby T, Adén D, Meléndez J et al (2017) Chemical evolution of the Galactic bulge as traced by microlensed dwarf and subgiant stars. VI. Age and abundance structure of the stellar populations in the central sub-kpc of the Milky Way. A\&A 605:A89. https://doi.org/10.1051/0004-6361/ 201730560. arXiv:1702.02971 [astro-ph.GA]

Bernard EJ, Schultheis M, Di Matteo P et al (2018) Star formation history of the Galactic bulge from deep HST imaging of low reddening windows. MNRAS 477(3):3507-3519. https://doi.org/10.1093/ mnras/sty902. arXiv:1801.01426 [astro-ph.GA]

Bilitewski T, Schönrich R (2012) Radial flows and angular momentum conservation in Galactic chemical evolution. MNRAS 426(3):2266-2282. https://doi.org/10.1111/j.1365-2966.2012.21827.x. arXiv: 1208.0003 [astro-ph.GA]

Binney JJ, Sellwood JA (2000) Disk heating and stellar migration in galaxies. arXiv:astro-ph/0003194

Boissier S, Prantzos N (1999) Chemo-spectrophotometric evolution of spiral galaxies-I. The model and the Milky Way. MNRAS 307(4):857-876. https://doi.org/10.1046/j.1365-8711.1999.02699.x. arXiv:astro-ph/9902148

Bonifacio P, Molaro P (1997) The primordial lithium abundance. MNRAS 285(4):847-861. https://doi. org/10.1093/mnras/285.4.847. arXiv:astro-ph/9611043

Bonifacio P, Caffau E, Spite M et al (2015) TOPoS II On the bimodality of carbon abundance in CEMP stars Implications on the early chemical evolution of galaxies. A\&A 579:A28. https://doi.org/10. 1051/0004-6361/201425266. arXiv:1504.05963 [astro-ph.GA]

Brook CB, Stinson GS, Gibson BK et al (2012) Thin disc, thick disc and halo in a simulated galaxy. MNRAS 426(1):690-700. https://doi.org/10.1111/j.1365-2966.2012.21738.x. arXiv:1206.0740 [astro-ph.GA] 
Brusadin G, Matteucci F, Romano D (2013) Modeling the chemical evolution of the Galaxy halo. A\&A 554:A135. https://doi.org/10.1051/0004-6361/201220884. arXiv:1304.4385 [astro-ph.GA]

Buck T (2020) On the origin of the chemical bimodality of disc stars: a tale of merger and migration. MNRAS 491(4):5435-5446. https://doi.org/10.1093/mnras/stz3289. arXiv:1909.09162 [astroph.GA]

Bullock JS, Kravtsov AV, Weinberg DH (2001) Hierarchical galaxy formation and substructure in the Galaxy's stellar halo. ApJ 548(1):33-46. https://doi.org/10.1086/318681. arXiv:astro-ph/0007295

Busso M, Gallino R, Wasserburg GJ (1999) Nucleosynthesis in asymptotic giant branch stars: relevance for galactic enrichment and solar system formation. ARA\&A 37:239-309. https://doi.org/10.1146/ annurev.astro.37.1.239

Calura F, Menci N (2009) Chemical evolution of local galaxies in a hierarchical model. MNRAS 400(3):1347-1365. https://doi.org/10.1111/j.1365-2966.2009.15440.x. arXiv:0907.3729 [astroph.CO]

Calura F, Menci N (2011) A possible solution to the $[\alpha / \mathrm{Fe}]-\sigma$ problem in early-type galaxies within a hierarchical galaxy formation model. MNRAS 413(1):L1-L5. https://doi.org/10.1111/j.1745-3933. 2011.01017.x. arXiv:1101.4938 [astro-ph.CO]

Carollo D, Beers TC, Lee YS et al (2007) Two stellar components in the halo of the Milky Way. Nature 450(7172):1020-1025. https://doi.org/10.1038/nature06460. arXiv:0706.3005 [astro-ph]

Carollo D, Beers TC, Bovy J et al (2012) Carbon-enhanced metal-poor stars in the inner and outer halo components of the milky way. ApJ 744(2):195. https://doi.org/10.1088/0004-637X/744/2/195. arXiv:1103.3067 [astro-ph.GA]

Cavallo L, Cescutti G, Matteucci F (2021) Neutron stars mergers in a stochastic chemical evolution model: impact of time delay distributions. MNRAS 503(1):1-12. https://doi.org/10.1093/mnras/ stab281. arXiv:2009.09534 [astro-ph.GA]

Cavichia O, Mollá M, Costa RDD, Maciel WJ (2014) The role of the Galactic bar in the chemical evolution of the Milky Way. MNRAS 437(4):3688-3701. https://doi.org/10.1093/mnras/stt2164. arXiv:1311.1518 [astro-ph.GA]

Cayrel R, Depagne E, Spite M et al (2004) First stars V-abundance patterns from C to Zn and supernova yields in the early Galaxy. A\&A 416:1117-1138. https://doi.org/10.1051/0004-6361:20034074. arXiv:astro-ph/0311082

Cen R, Ostriker JP (1999) Cosmic chemical evolution. ApJ 519(2):L109-L113. https://doi.org/10.1086/ 312123. arXiv:astro-ph/9903207

Cescutti G (2008) An inhomogeneous model for the Galactic halo: a possible explanation for the spread observed in s- and r-process elements. A\&A 481(3):691-699. https://doi.org/10.1051/0004-6361: 20078571. arXiv:0802.0678 [astro-ph]

Cescutti G, Chiappini C (2014) Explaining the Ba, Y, Sr, and Eu abundance scatter in metal-poor halo stars: constraints to the r-process. A\&A 565:A51. https://doi.org/10.1051/0004-6361/201423432. arXiv:1403.8070 [astro-ph.GA]

Cescutti G, Matteucci F (2011) Galactic astroarchaeology: reconstructing the bulge history by means of the newest data. A\&A 525:A126. https://doi.org/10.1051/0004-6361/201015665. arXiv:1010.1469 [astro-ph.GA]

Cescutti G, François P, Matteucci F, Cayrel R, Spite M (2006) The chemical evolution of barium and europium in the Milky Way. A\&A 448(2):557-569. https://doi.org/10.1051/0004-6361:20053622. arXiv:astro-ph/0510496

Cescutti G, Matteucci F, François P, Chiappini C (2007) Abundance gradients in the Milky Way for $\alpha$ elements, iron peak elements, barium, lanthanum, and europium. A\&A 462(3):943-951. https://doi. org/10.1051/0004-6361:20065403. arXiv:astro-ph/0609813

Cescutti G, Matteucci F, Lanfranchi GA, McWilliam A (2008) The chemical evolution of manganese in different stellar systems. A\&A 491(2):401-405. https://doi.org/10.1051/0004-6361:200810537. arXiv:0807.1463 [astro-ph]

Cescutti G, Chiappini C, Hirschi R, Meynet G, Frischknecht U (2013) The s-process in the Galactic halo: the fifth signature of spinstars in the early Universe? A\&A 553:A51. https://doi.org/10.1051/00046361/201220809. arXiv:1302.4354 [astro-ph.GA]

Cescutti G, Romano D, Matteucci F, Chiappini C, Hirschi R (2015) The role of neutron star mergers in the chemical evolution of the Galactic halo. A\&A 577:A139. https://doi.org/10.1051/0004-6361/ 201525698. arXiv:1503.02954 [astro-ph.GA]

Cescutti G, Chiappini C, Hirschi R (2018) The oldest stars of the bulge: new information on the ancient Galaxy. In: Chiappini C, Minchev I, Starkenburg E, Valentini M (eds) Rediscovering our galaxy. 
Proceedings IAU, vol 334. Cambridge University Press, Cambridge, pp 94-97. https://doi.org/10. 1017/S1743921317008183. arXiv:1710.11014 [astro-ph.GA]

Chabrier G (2003) Galactic stellar and substellar initial mass function. PASP 115(809):763-795. https:// doi.org/10.1086/376392. arXiv:astro-ph/0304382

Charbonnel C, Lagarde N (2010) Thermohaline instability and rotation-induced mixing. I. Low- and intermediate-mass solar metallicity stars up to the end of the AGB. A\&A 522:A10. https://doi.org/ 10.1051/0004-6361/201014432. arXiv:1006.5359 [astro-ph.SR]

Chiappini C (2009) The chemical evolution of the galactic thick and thin disks. In: Andersen J, Nordström B, Bland-Hawthorn J (eds) The galaxy disk in cosmological context, Proceedings IAU, vol 4 (S254). Cambridge University Press, Cambridge, pp 191-196. https://doi.org/10.1017/ S1743921308027580

Chiappini C, Matteucci F, Gratton R (1997) The chemical evolution of the galaxy: the two-infall model. ApJ 477(2):765-780. https://doi.org/10.1086/303726. arXiv:astro-ph/9609199

Chiappini C, Matteucci F, Romano D (2001) Abundance gradients and the formation of the Milky Way. ApJ 554(2):1044-1058. https://doi.org/10.1086/321427. arXiv:astro-ph/0102134

Chiappini C, Hirschi R, Meynet G et al (2006) A strong case for fast stellar rotation at very low metallicities. A\&A 449(2):L27-L30. https://doi.org/10.1051/0004-6361:20064866. arXiv:astro-ph/ 0602459

Chiappini C, Frischknecht U, Meynet G et al (2011) Imprints of fast-rotating massive stars in the Galactic Bulge. Nature 472(7344):454-457. https://doi.org/10.1038/nature10000

Chiosi C (1980) Chemical evolution of the galactic disk: the inflow problem. A\&A 83:206-216

Clarke JP, Wegg C, Gerhard O et al (2019) The Milky Way bar/bulge in proper motions: a 3D view from VIRAC and Gaia. MNRAS 489(3):3519-3538. https://doi.org/10.1093/mnras/stz2382. arXiv:1903. 02003 [astro-ph.GA]

Clarkson WI, Sahu KC, Anderson J et al (2011) The first detection of blue straggler stars in the Milky Way bulge. ApJ 735(1):37. https://doi.org/10.1088/0004-637X/735/1/37. arXiv:1105.4176 [astroph.GA]

Clegg RES, Lambert DL, Tomkin J (1981) Carbon, nitrogen and oxygen abundances in main-sequence stars. II. 20F and G stars. ApJ 250:262-275. https://doi.org/10.1086/159371

Coc A, Uzan JP, Vangioni E (2014) Standard big bang nucleosynthesis and primordial CNO abundances after Planck. J Cosmol Astropart Phys 10:050. https://doi.org/10.1088/1475-7516/2014/10/050. arXiv:1403.6694 [astro-ph.CO]

Colavitti E, Matteucci F, Murante G (2008) The chemical evolution of a Milky Way-like galaxy: the importance of a cosmologically motivated infall law. A\&A 483(2):401-413. https://doi.org/10.1051/ 0004-6361:200809413. arXiv:0802.1847 [astro-ph]

Colavitti E, Cescutti G, Matteucci F, Murante G (2009) The origin of abundance gradients in the Milky Way: the predictions of different models. A\&A 496(2):429-439. https://doi.org/10.1051/0004-6361: 200810891. arXiv:0811.3505 [astro-ph]

Cole S, Aragon-Salamanca A, Frenk CS, Navarro JF, Zepf SE (1994) A recipe for galaxy formation. MNRAS 271:781-806. https://doi.org/10.1093/mnras/271.4.781. arXiv:astro-ph/9402001

Cooke RJ, Fumagalli M (2018) Measurement of the primordial helium abundance from the intergalactic medium. Nat Astron 2:957-961. https://doi.org/10.1038/s41550-018-0584-z. arXiv:1810.06561 [astro-ph.CO]

Côté B, Fryer CL, Belczynski K et al (2018) The origin of r-process elements in the Milky Way. ApJ 855(2):99. https://doi.org/10.3847/1538-4357/aaad67. arXiv:1710.05875 [astro-ph.GA]

Côté B, Eichler M, Arcones A et al (2019) Neutron star mergers might not be the only source of r-process elements in the Milky Way. ApJ 875(2):106. https://doi.org/10.3847/1538-4357/ab10db. arXiv: 1809.03525 [astro-ph.HE]

Cowan JJ, Thielemann FK, Truran JW (1991) The R-process and nucleochronology. Phys Rep 208(4-5):267-394. https://doi.org/10.1016/0370-1573(91)90070-3

Cristallo S, Straniero O, Gallino R et al (2009) Evolution, nucleosynthesis, and yields of low-mass asymptotic giant branch stars at different metallicities. ApJ 696(1):797-820. https://doi.org/10.1088/ 0004-637X/696/1/797. arXiv:0902.0243 [astro-ph.SR]

Cristallo S, Piersanti L, Straniero O et al (2011) Evolution, nucleosynthesis, and yields of low-mass asymptotic giant branch stars at different metallicities. II. The FRUITY Database. ApJS 197(2):17. https://doi.org/10.1088/0067-0049/197/2/17. arXiv:1109.1176 [astro-ph.SR]

D’Antona F, Matteucci F (1991) Galactic evolution of lithium. A\&A 248:62 
de Laverny P, Recio-Blanco A, Worley CC et al (2013) The AMBRE Project: stellar parameterisation of ESO archived spectra. Messenger 153:18-21

De Lucia G, Helmi A (2008) The Galaxy and its stellar halo: insights on their formation from a hybrid cosmological approach. MNRAS 391(1):14-31. https://doi.org/10.1111/j.1365-2966.2008.13862.x. arXiv:0804.2465 [astro-ph]

De Silva GM, Freeman KC, Bland-Hawthorn J et al (2015) The GALAH survey: scientific motivation. MNRAS 449(3):2604-2617. https://doi.org/10.1093/mnras/stv327. arXiv:1502.04767 [astro-ph.GA]

Dearborn DSP, Steigman G, Tosi M (1996) Galactic evolution of D and 3He including stellar production of 3He. ApJ 465:887. https://doi.org/10.1086/177472. arXiv:astro-ph/9601117

Debattista VP, Ness M, Gonzalez OA et al (2017) Separation of stellar populations by an evolving bar: implications for the bulge of the Milky Way. MNRAS 469(2):1587-1611. https://doi.org/10.1093/ mnras/stx947. arXiv:1611.09023 [astro-ph.GA]

Della Valle M, Izzo L (2020) Observations of galactic and extragalactic novae. A\&A Rev 28(1):3. https:// doi.org/10.1007/s00159-020-0124-6. arXiv:2004.06540 [astro-ph.SR]

Di Matteo P, Gómez A, Haywood M et al (2015) Why the Milky Way's bulge is not only a bar formed from a cold thin disk. A\&A 577:A1. https://doi.org/10.1051/0004-6361/201424457. arXiv:1411. 1416 [astro-ph.GA]

Diaz AI, Tosi M (1986) The origin of nitrogen and the chemical evolution of spiral galaxies. A\&A 158:60-66

Domínguez-Tenreiro R, Obreja A, Brook CB, Martínez-Serrano FJ, Serna A (2017) The radial distribution of mono-metallicity populations in the galactic disk as evidence for two-phase disk formation. ApJ 846(1):72. https://doi.org/10.3847/1538-4357/aa77af. arXiv:1706.05031 [astroph.GA]

Eggen OJ, Lynden-Bell D, Sandage AR (1962) Evidence from the motions of old stars that the Galaxy collapsed. ApJ 136:748. https://doi.org/10.1086/147433

Eichler M, Arcones A, Kelic A et al (2015) The role of fission in neutron star mergers and its impact on the r-process peaks. ApJ 808(1):30. https://doi.org/10.1088/0004-637X/808/1/30. arXiv:1411.0974 [astro-ph.HE]

Evans PA, Cenko SB, Kennea JA et al (2017) Swift and NuSTAR observations of GW170817: detection of a blue kilonova. Science 358(6370):1565-1570. https://doi.org/10.1126/science.aap9580. arXiv: 1710.05437 [astro-ph.HE]

Few CG, Courty S, Gibson BK, Michel-Dansac L, Calura F (2014) Chemodynamics of a simulated disc galaxy: initial mass functions and Type Ia supernova progenitors. MNRAS 444(4):3845-3862. https://doi.org/10.1093/mnras/stu1709. arXiv:1408.5135 [astro-ph.GA]

Font AS, Johnston KV, Bullock JS, Robertson B (2005) Galactic chemical abundance distributions in a $\Lambda$ CDM universe. In: Jerjen H, Binggeli B (eds) IAU Colloq. 198: Near-fields cosmology with dwarf elliptical galaxies. Cambridge University Press, Cambridge, pp 126-129. https://doi.org/10.1017/ S1743921305003613

François P (1986) Chemical evolution of the galaxy: a comparison of the abundances of light metals in disk and halo dwarfs. A\&A 160:264-276

François P, Matteucci F, Cayrel R et al (2004) The evolution of the Milky Way from its earliest phases: constraints on stellar nucleosynthesis. A\&A 421:613-621. https://doi.org/10.1051/0004-6361: 20034140. arXiv:astro-ph/0401499

Francois P, Matteucci F (1993) On the abundance spread in solar neighbourhood stars. A\&A 280(1):136-140

Freeman K, Ness M, Wylie-de-Boer E et al (2013) ARGOS-II. The Galactic bulge survey. MNRAS 428(4):3660-3670. https://doi.org/10.1093/mnras/sts305. arXiv:1212.1541 [astro-ph.GA]

Freiburghaus C, Rosswog S, Thielemann FK (1999) R-process in neutron star mergers. ApJ 525(2):L121L124. https://doi.org/10.1086/312343

Frischknecht U, Hirschi R, Thielemann FK (2012) Non-standard s-process in low metallicity massive rotating stars. A\&A 538:L2. https://doi.org/10.1051/0004-6361/201117794. arXiv:1112.5548 [astroph.SR]

Fu X, Romano D, Bragaglia A et al (2018) The Gaia-ESO survey: lithium enrichment histories of the galactic thick and thin disc. A\&A 610:A38. https://doi.org/10.1051/0004-6361/201731677. arXiv: 1711.04829 [astro-ph.GA]

Fuhrmann K (1998) Nearby stars of the Galactic disk and halo. A\&A 338:161-183

Fuhrmann K (2011) Nearby stars of the Galactic disc and halo - V. MNRAS 414(4):2893-2922. https:// doi.org/10.1111/j.1365-2966.2011.18476.x 
Galli D, Palla F, Ferrini F, Penco U (1995) Galactic evolution of D and ${ }^{3}$ He. ApJ 443:536. https://doi.org/ $10.1086 / 175546$

Gao S, Liu C, Zhang X et al (2014) The binarity of Milky Way F, G, K stars as a function of effective temperature and metallicity. ApJ 788(2):L37. https://doi.org/10.1088/2041-8205/788/2/L37. arXiv: 1405.7105 [astro-ph.GA]

Gao S, Zhao H, Yang H, Gao R (2017) The binarity of Galactic dwarf stars along with effective temperature and metallicity. MNRAS 469(1):L68-L72. https://doi.org/10.1093/mnras1/slx048. arXiv:1703.10305 [astro-ph.SR]

Geisler D, Wallerstein G, Smith VV, Casetti-Dinescu DI (2007) Chemical abundances and kinematics in globular clusters and local group dwarf galaxies and their implications for formation theories of the galactic halo. PASP 119(859):939-961. https://doi.org/10.1086/521990. arXiv:0708.0570 [astro-ph]

Geiss J, Gloeckler G (1998) Abundances of deuterium and helium-3 in the protosolar cloud. Space Sci Rev 84:239-250. https://doi.org/10.1023/A:1005039822524

Ghirlanda G, Salafia OS, Pescalli A et al (2016) Short gamma-ray bursts at the dawn of the gravitational wave era. A\&A 594:A84. https://doi.org/10.1051/0004-6361/201628993. arXiv:1607.07875 [astroph.HE]

Gilmore G, Reid N (1983) New light on faint stars - III. Galactic structure towards the South Pole and the Galactic thick disc. MNRAS 202:1025-1047. https://doi.org/10.1093/mnras/202.4.1025

Gilmore G, Randich S, Asplund M et al (2012) The Gaia-ESO public spectroscopic survey. Messenger 147:25-31

Gilmore G, Norris JE, Monaco L et al (2013) Elemental abundances and their implications for the chemical enrichment of the Boötes I ultrafaint galaxy. ApJ 763(1):61. https://doi.org/10.1088/0004637X/763/1/61. arXiv:1212.0598 [astro-ph.GA]

Goetz M, Koeppen J (1992) On abundance gradients in spiral galaxies. A\&A 262(2):455-467

Grand RJJ, Gómez FA, Marinacci F et al (2017) The Auriga Project: the properties and formation mechanisms of disc galaxies across cosmic time. MNRAS 467(1):179-207. https://doi.org/10.1093/ mnras/stx071. arXiv:1610.01159 [astro-ph.GA]

Grand RJJ, Bustamante S, Gómez FA et al (2018) Origin of chemically distinct discs in the Auriga cosmological simulations. MNRAS 474(3):3629-3639. https://doi.org/10.1093/mnras/stx3025. arXiv:1708.07834 [astro-ph.GA]

Grand RJJ, Kawata D, Belokurov V et al (2020) The dual origin of the Galactic thick disc and halo from the gas-rich Gaia-Enceladus Sausage merger. MNRAS 497:1603

Gratton R, Carretta E, Matteucci F, Sneden C (1996) The [Fe/O] ratio in field stars and the history of star formation of the solar neighbourhood. In: Morrison HL, Sarajedini A (eds) Formation of the galactic halo...inside and out. ASP conference series, vol 92. Astronomical Society of the Pacific, p 307

Gratton RG, Carretta E, Matteucci F, Sneden C (2000) Abundances of light elements in metal-poor stars. IV. $[\mathrm{Fe} / \mathrm{O}]$ and $[\mathrm{Fe} / \mathrm{Mg}]$ ratios and the history of star formation in the solar neighborhood. A\&A 358:671-681. arXiv:astro-ph/0004157

Gratton RG, Carretta E, Desidera S et al (2003) Abundances for metal-poor stars with accurate parallaxes. II. alpha-elements in the halo. A\&A 406:131-140. https://doi.org/10.1051/0004-6361:20030754. arXiv:astro-ph/0305358

Greggio L (2005) The rates of type Ia supernovae I. Analytical formulations. A\&A 441(3):1055-1078. https://doi.org/10.1051/0004-6361:20052926. arXiv:astro-ph/0504376

Greggio L, Renzini A (1983) Iron versus oxygen production-the role of type I supernovae. Mem Soc Astron Ital 54(1):311-319

Grenon M (1989) The chemical evolution of the galactic disc from the kinematics and metallicities of proper-motion stars. Ap\&SS 156(1-2):29-37. https://doi.org/10.1007/BF00646341

Grieco V, Matteucci F, Meynet G et al (2012a) Metallicity effects on cosmic Type Ib/c supernovae and gamma-ray burst rates. MNRAS 423(4):3049-3057. https://doi.org/10.1111/j.1365-2966.2012. 21052.x

Grieco V, Matteucci F, Pipino A, Cescutti G (2012b) Chemical evolution of the Galactic bulge: different stellar populations and possible gradients. A\&A 548:A60. https://doi.org/10.1051/0004-6361/ 201219761. arXiv:1209.4462 [astro-ph.GA]

Grieco V, Matteucci F, Ryde N, Schultheis M, Uttenthaler S (2015) Chemical evolution of the Galactic Centre. MNRAS 450(2):2094-2103. https://doi.org/10.1093/mnras/stv729. arXiv:1503.08949 [astro-ph.GA] 
Grisoni V, Spitoni E, Matteucci F et al (2017) The AMBRE project: chemical evolution models for the Milky Way thick and thin discs. MNRAS 472(3):3637-3647. https://doi.org/10.1093/mnras/ stx2201. arXiv:1706.02614 [astro-ph.GA]

Grisoni V, Spitoni E, Matteucci F (2018) Abundance gradients along the Galactic disc from chemical evolution models. MNRAS 481(2):2570-2580. https://doi.org/10.1093/mnras/sty2444. arXiv:1805. 11415 [astro-ph.GA]

Grisoni V, Matteucci F, Romano D, Fu X (2019) Evolution of lithium in the Milky Way halo, discs, and bulge. MNRAS 489(3):3539-3546. https://doi.org/10.1093/mnras/stz2428. arXiv:1906.09130 [astro-ph.GA]

Guiglion G, de Laverny P, Recio-Blanco A et al (2016) The AMBRE project: constraining the lithium evolution in the Milky Way. A\&A 595:A18. https://doi.org/10.1051/0004-6361/201628919. arXiv: 1608.03411 [astro-ph.GA]

Guiglion G, Chiappini C, Romano D et al (2019) Explaining the decrease in ISM lithium at super-solar metallicities in the solar vicinity. A\&A 623:A99. https://doi.org/10.1051/0004-6361/201834203. arXiv:1902.01660 [astro-ph.GA]

Hachisu I, Kato M, Nomoto K, Umeda H (1999) A new evolutionary path to type Ia supernovae: a helium-rich supersoft X-ray source channel. ApJ 519(1):314-323. https://doi.org/10.1086/307370. arXiv:astro-ph/9902303

Han Z, Podsiadlowski P (2004) The single-degenerate channel for the progenitors of Type Ia supernovae. MNRAS 350(4):1301-1309. https://doi.org/10.1111/j.1365-2966.2004.07713.x. arXiv:astro-ph/ 0309618

Hansen T, Hansen CJ, Christlieb N et al (2014) Exploring the origin of lithium, carbon, strontium, and barium with four new ultra metal-poor stars. ApJ 787(2):162. https://doi.org/10.1088/0004-637X/ 787/2/162. arXiv:1405.5846 [astro-ph.SR]

Hartwick FDA (1976) The chemical evolution of the galactic halo. ApJ 209:418-423. https://doi.org/10. $1086 / 154735$

Hayden MR, Bovy J, Holtzman JA et al (2015) Chemical cartography with APOGEE: metallicity distribution functions and the chemical structure of the Milky Way disk. ApJ 808(2):132. https://doi. org/10.1088/0004-637X/808/2/132. arXiv:1503.02110 [astro-ph.GA]

Haynes CJ, Kobayashi C (2019) Galactic simulations of r-process elemental abundances. MNRAS 483(4):5123-5134. https://doi.org/10.1093/mnras/sty3389. arXiv:1809.10991 [astro-ph.GA]

Haywood M, Di Matteo P, Snaith O, Calamida A (2016) Hiding its age: the case for a younger bulge. A\&A 593:A82. https://doi.org/10.1051/0004-6361/201628816. arXiv:1606.04092 [astro-ph.GA]

Helmi A (2008) The stellar halo of the Galaxy. A\&A Rev 15(3):145-188. https://doi.org/10.1007/s00159008-0009-6. arXiv:0804.0019 [astro-ph]

Helmi A, White SDM (1999) Building up the stellar halo of the Galaxy. MNRAS 307(3):495-517. https:// doi.org/10.1046/j.1365-8711.1999.02616.x. arXiv:astro-ph/9901102

Helmi A, White SDM, de Zeeuw PT, Zhao H (1999) Debris streams in the solar neighbourhood as relicts from the formation of the Milky Way. Nature 402(6757):53-55. https://doi.org/10.1038/46980. arXiv:astro-ph/9911041

Helmi A, Babusiaux C, Koppelman HH et al (2018) The merger that led to the formation of the Milky Way's inner stellar halo and thick disk. Nature 563(7729):85-88. https://doi.org/10.1038/s41586018-0625-x. arXiv:1806.06038 [astro-ph.GA]

Hill V, Lecureur A, Gómez A et al (2011) The metallicity distribution of bulge clump giants in Baade's window. A\&A 534:A80. https://doi.org/10.1051/0004-6361/200913757. arXiv:1107.5199 [astroph.GA]

Hillebrandt W, Kromer M, Röpke FK, Ruiter AJ (2013) Towards an understanding of Type Ia supernovae from a synthesis of theory and observations. Front Phys 8(2):116-143. https://doi.org/10.1007/ s11467-013-0303-2. arXiv:1302.6420 [astro-ph.CO]

Hoopes CG, Sembach KR, Hébrard G, Moos HW, Knauth DC (2003) Deuterium toward two Milky Way disk stars: probing extended sight lines with the far ultraviolet spectroscopic explorer. ApJ 586(2):1094-1110. https://doi.org/10.1086/367890. arXiv:astro-ph/0212303

Iben I Jr, Tutukov AV (1984) Supernovae of type I as end products of the evolution of binaries with components of moderate initial mass. ApJS 54:335-372. https://doi.org/10.1086/190932

Immeli A, Samland M, Gerhard O, Westera P (2004) Gas physics, disk fragmentation, and bulge formation in young galaxies. A\&A 413:547-561. https://doi.org/10.1051/0004-6361:20034282. arXiv:astro-ph/0312139 
Iorio G, Belokurov V (2021) Chemo-kinematics of the Gaia RR Lyrae: the halo and the disc. MNRAS. https://doi.org/10.1093/mnras/stab005. arXiv:2008.02280 [astro-ph.GA]

Iwamoto K, Brachwitz F, Nomoto K et al (1999) Nucleosynthesis in Chandrasekhar mass models for type Ia supernovae and constraints on progenitor systems and burning-front propagation. ApJS 125(2):439-462. https://doi.org/10.1086/313278. arXiv:astro-ph/0002337

Izotov YI, Thuan TX, Guseva NG (2014) A new determination of the primordial He abundance using the He I $\lambda 10830 \AA$ emission line: cosmological implications. MNRAS 445(1):778-793. https://doi.org/ 10.1093/mnras/stu1771. arXiv:1408.6953 [astro-ph.CO]

Izzo L, Della Valle M, Mason E et al (2015) Early optical spectra of Nova V1369 Cen show the presence of lithium. ApJ 808(1):L14. https://doi.org/10.1088/2041-8205/808/1/L14. arXiv:1506.08048 [astroph.SR]

Izzo L, Molaro P, Bonifacio P et al (2018) Beryllium detection in the very fast nova ASASSN-16kt (V407 Lupi). MNRAS 478(2):1601-1610. https://doi.org/10.1093/mnras/sty435. arXiv:1802.05896 [astroph.SR]

Jacq T, Baudry A, Walmsley CM, Caselli P (1999) Deuterium in the Sagittarius B2 and Sagittarius A galactic center regions. A\&A 347:957-966

Jeans JH (1902) The stability of a spherical nebula. Philos Trans R Soc Lond Ser A 199:1-53. https://doi. org/10.1098/rsta.1902.0012

Jenkins EB, Oegerle WR, Sembach KR, et al. (1999) A new insight on the ionization of the local interstellar medium, as revealed by FUSE observations of Ar I toward White Dwarf Stars. In: 195th AAS meeting. American Astronomical Society Meeting Abstracts, vol 195, p 06.05

Jiménez N, Tissera PB, Matteucci F (2015) Type Ia supernova progenitors and chemical enrichment in hydrodynamical simulations. I. The single-degenerate scenario. ApJ 810(2):137. https://doi.org/10. 1088/0004-637X/810/2/137. arXiv:1402.4137 [astro-ph.GA]

Johnson CI, Rich RM, Fulbright JP, Valenti E, McWilliam A (2011) Alpha enhancement and the metallicity distribution function of Plaut's window. ApJ 732(2):108. https://doi.org/10.1088/0004637X/732/2/108. arXiv:1103.2143 [astro-ph.SR]

Johnson CI, Rich RM, Kobayashi C, Fulbright JP (2012) Constraints on the formation of the galactic bulge from $\mathrm{Na}, \mathrm{Al}$, and heavy-element abundances in Plaut's field. ApJ 749(2):175. https://doi.org/ 10.1088/0004-637X/749/2/175. arXiv:1202.4481 [astro-ph.SR]

Johnson CI, Rich RM, Kobayashi C, Kunder A, Koch A (2014) Light, alpha, and Fe-peak element abundances in the galactic bulge. AJ 148(4):67. https://doi.org/10.1088/0004-6256/148/4/67. arXiv: 1407.2282 [astro-ph.SR]

Johnston KV, Hernquist L, Bolte M (1996) Fossil signatures of ancient accretion events in the Halo. ApJ 465:278. https://doi.org/10.1086/177418. arXiv:astro-ph/9602060

José J, Hernanz M (2007) Nucleosynthesis in classical nova explosions. J Phys G 34(12):R431-R458. https://doi.org/10.1088/0954-3899/34/12/R01

José J, Hernanz M, García Berro E (2003) The impact of the chemical stratification of white dwarfs on the nucleosynthesis from classical novae. Nucl Phys A 718:255-258. https://doi.org/10.1016/S03759474(03)00723-1

Kalogera V, Kim C, Lorimer DR et al (2004) The cosmic coalescence rates for double neutron star binaries. ApJ 601(2):L179-L182. https://doi.org/10.1086/382155

Karakas AI (2010) Updated stellar yields from asymptotic giant branch models. MNRAS 403(3):1413-1425. https://doi.org/10.1111/j.1365-2966.2009.16198.x. arXiv:0912.2142 [astroph.SR]

Karakas AI, Lugaro M (2016) Stellar yields from metal-rich asymptotic giant branch models. ApJ 825(1):26. https://doi.org/10.3847/0004-637X/825/1/26. arXiv:1604.02178 [astro-ph.SR]

Katz N (1992) Dissipational galaxy formation. II. Effects of star formation. ApJ 391:502. https://doi.org/ $10.1086 / 171366$

Kauffmann G, White SDM, Guiderdoni B (1993) The formation and evolution of galaxies within merging dark matter haloes. MNRAS 264:201-218. https://doi.org/10.1093/mnras/264.1.201

Kawata D, Gibson BK (2003) GCD+: a new chemodynamical approach to modelling supernovae and chemical enrichment in elliptical galaxies. MNRAS 340(3):908-922. https://doi.org/10.1046/j.13658711.2003.06356.x. arXiv:astro-ph/0212401

Kawata D, Gibson BK (2003) Multiwavelength cosmological simulations of elliptical galaxies. MNRAS 346(1):135-152. https://doi.org/10.1046/j.1365-2966.2003.07080.x. arXiv:astro-ph/0308168

Kennicutt J, Robert C (1989) The star formation law in galactic disks. ApJ 344:685. https://doi.org/10. $1086 / 167834$ 
Kennicutt J, Robert C (1998) The global Schmidt law in star-forming galaxies. ApJ 498(2):541-552. https://doi.org/10.1086/305588. arXiv:astro-ph/9712213

Khoperskov S, Di Matteo P, Haywood M, Gómez A, Snaith ON (2020) Escapees from the bar resonances. Presence of low-eccentricity metal-rich stars at the solar vicinity. A\&A 638:A144. https://doi.org/ 10.1051/0004-6361/201937188. arXiv:1911.12424 [astro-ph.GA]

Kobayashi C (2004) GRAPE-SPH chemodynamical simulation of elliptical galaxies - I. Evolution of metallicity gradients. MNRAS 347(3):740-758. https://doi.org/10.1111/j.1365-2966.2004.07258.x. arXiv:astro-ph/0310160

Kobayashi C, Nakasato N (2011) Chemodynamical simulations of the Milky Way galaxy. ApJ 729(1):16. https://doi.org/10.1088/0004-637X/729/1/16. arXiv:1012.5144 [astro-ph.GA]

Kobayashi C, Nomoto K (2009) The role of type Ia supernovae in chemical evolution I lifetime of type Ia supernovae and metallicity effect. ApJ 707(2):1466-1484. https://doi.org/10.1088/0004-637X/707/ 2/1466. arXiv:0801.0215 [astro-ph]

Kobayashi C, Umeda H, Nomoto K, Tominaga N, Ohkubo T (2006) Galactic chemical evolution: carbon through zinc. ApJ 653(2):1145-1171. https://doi.org/10.1086/508914. arXiv:astro-ph/0608688

Kobayashi C, Springel V, White SDM (2007) Simulations of cosmic chemical enrichment. MNRAS 376(4):1465-1479. https://doi.org/10.1111/j.1365-2966.2007.11555.x. arXiv:astro-ph/0604107

Kobayashi C, Karakas AI, Umeda H (2011) The evolution of isotope ratios in the Milky Way Galaxy. MNRAS 414(4):3231-3250. https://doi.org/10.1111/j.1365-2966.2011.18621.x. arXiv:1102.5312 [astro-ph.GA]

Kobayashi C, Karakas AI, Lugaro M (2020a) The origin of elements from carbon to uranium. ApJ 900(2):179. https://doi.org/10.3847/1538-4357/abae65. arXiv:2008.04660 [astro-ph.GA]

Kobayashi C, Leung SC, Nomoto K (2020b) New type Ia supernova yields and the manganese and nickel problems in the Milky Way and dwarf spheroidal galaxies. ApJ 895(2):138. https://doi.org/10.3847/ 1538-4357/ab8e44. arXiv:1906.09980 [astro-ph.HE]

Komiya Y, Shigeyama T (2016) Contribution of neutron star mergers to the r-process chemical evolution in the hierarchical galaxy formation. ApJ 830(2):76. https://doi.org/10.3847/0004-637X/830/2/76. arXiv:1608.01772 [astro-ph.GA]

Korobkin O, Rosswog S, Arcones A, Winteler C (2012) On the astrophysical robustness of the neutron star merger r-process. MNRAS 426(3):1940-1949. https://doi.org/10.1111/j.1365-2966.2012.21859. x. arXiv:1206.2379 [astro-ph.SR]

Kroupa P (2001) On the variation of the initial mass function. MNRAS 322(2):231-246. https://doi.org/ 10.1046/j.1365-8711.2001.04022.x. arXiv:astro-ph/0009005

Kroupa P, Tout CA, Gilmore G (1993) The distribution of low-mass stars in the galactic disc. MNRAS 262:545-587. https://doi.org/10.1093/mnras/262.3.545

Kubryk M, Prantzos N, Athanassoula E (2015) Evolution of the Milky Way with radial motions of stars and gas I. The solar neighbourhood and the thin and thick disks. A\&A 580:A126. https://doi.org/10. 1051/0004-6361/201424171. arXiv:1412.0585 [astro-ph.GA]

Kuijken K, Gilmore G (1989) The mass distribution in the galactic disc - II. Determination of the surface mass density of the galactic disc near the Sun. MNRAS 239:605-649. https://doi.org/10.1093/ mnras/239.2.605

Kunder A, Koch A, Rich RM, de Propris R, Howard CD, Stubbs SA, Johnson CI, Shen J, Wang Y, Robin AC, Kormendy J, Soto M, Frinchaboy P, Reitzel DB, Zhao H, Origlia L (2012) The bulge radial velocity assay (BRAVA). II. Complete sample and data release. Astron J 143(3):57. https://doi.org/ 10.1088/0004-6256/143/3/57. arXiv:1112.1955 [astro-ph.SR]

Lacey CG, Fall SM (1985) Chemical evolution of the galactic disk with radial gas flows. ApJ 290:154-170. https://doi.org/10.1086/162970

Lagarde N, Romano D, Charbonnel C et al (2012) Effects of thermohaline instability and rotationinduced mixing on the evolution of light elements in the Galaxy: D, ${ }^{3} \mathrm{He}$ and ${ }^{4} \mathrm{He}$. A\&A 542:A62. https://doi.org/10.1051/0004-6361/201219132. arXiv:1204.2266 [astro-ph.SR]

Larson RB (1972) Effect of infalling matter on the heavy element content of a galaxy. Nat Phys Sci 236(62):7-8. https://doi.org/10.1038/physci236007a0

Larson RB (1976) Models for the formation of disc galaxies. MNRAS 176:31-52. https://doi.org/10. 1093/mnras/176.1.31

Lattimer JM, Mackie F, Ravenhall DG, Schramm DN (1977) The decompression of cold neutron star matter. ApJ 213:225-233. https://doi.org/10.1086/155148 
Leung HW, Bovy J (2019) Simultaneous calibration of spectro-photometric distances and the Gaia DR2 parallax zero-point offset with deep learning. MNRAS 489(2):2079-2096. https://doi.org/10.1093/ mnras/stz2245. arXiv:1902.08634 [astro-ph.GA]

Lilly SJ, Carollo CM, Pipino A, Renzini A, Peng Y (2013) Gas regulation of galaxies: the evolution of the cosmic specific star formation rate, the metallicity-mass-star-formation rate relation, and the stellar content of halos. ApJ 772(2):119. https://doi.org/10.1088/0004-637X/772/2/119. arXiv:1303.5059 [astro-ph.CO]

Limongi M, Chieffi A (2018) Presupernova evolution and explosive nucleosynthesis of rotating massive stars in the metallicity range $-3 \leq[\mathrm{Fe} / \mathrm{H}] \leq 0$. ApJS 237(1):13. https://doi.org/10.3847/1538-4365/ aacb24. arXiv:1805.09640 [astro-ph.SR]

Linsky JL (1998) Deuterium abundance in the local ISM and possible spatial variations. Space Sci Rev 84:285-296. https://doi.org/10.1023/A:1005048124341

Loebman SR, Debattista VP, Nidever DL et al (2016) Imprints of radial migration on the Milky Way's metallicity distribution functions. ApJL 818(1):L6. https://doi.org/10.3847/2041-8205/818/1/L6. arXiv:1511.06369 [astro-ph.GA]

Lubowich DA, Pasachoff JM, Balonek TJ et al (2000) Deuterium in the Galactic Centre as a result of recent infall of low-metallicity gas. Nature 405(6790):1025-1027. https://doi.org/10.1038/35016506

Lucey M, Hawkins K, Ness M et al (2019) The COMBS survey-I. Chemical origins of metal-poor stars in the Galactic bulge. MNRAS 488(2):2283-2300. https://doi.org/10.1093/mnras/stz1847. arXiv: 1903.11615 [astro-ph.SR]

Lynden-Bell D (1975) The chemical evolution of galaxies. Vistas Astron 19(3):299-316. https://doi.org/ 10.1016/0083-6656(75)90005-7

Mackereth JT, Bovy J (2020) Weighing the stellar constituents of the galactic halo with APOGEE red giant stars. MNRAS 492(3):3631-3646. https://doi.org/10.1093/mnras/staa047. arXiv:1910.03590 [astro-ph.GA]

Mackereth JT, Crain RA, Schiavon RP et al (2018) The origin of diverse $\alpha$-element abundances in galaxy discs. MNRAS 477(4):5072-5089. https://doi.org/10.1093/mnras/sty972. arXiv:1801.03593 [astroph.GA]

Maiolino R, Mannucci F (2019) De re metallica: the cosmic chemical evolution of galaxies. A\&A Rev 27(1):3. https://doi.org/10.1007/s00159-018-0112-2. arXiv:1811.09642 [astro-ph.GA]

Majewski SR, Schiavon RP, Frinchaboy PM et al (2017) The Apache Point Observatory Galactic Evolution Experiment (APOGEE). AJ 154(3):94. https://doi.org/10.3847/1538-3881/aa784d. arXiv: 1509.05420 [astro-ph.IM]

Mannucci F, Della Valle M, Panagia N (2006) Two populations of progenitors for Type Ia supernovae? MNRAS 370(2):773-783. https://doi.org/10.1111/j.1365-2966.2006.10501.x. arXiv:astro-ph/ 0510315

Maoz D, Mannucci F (2012) Type-Ia supernova rates and the progenitor problem: a review. PASA 29(4):447-465. https://doi.org/10.1071/AS11052. arXiv:1111.4492 [astro-ph.CO]

Martin CL, Kobulnicky HA, Heckman TM (2002) The metal content of dwarf starburst winds: results from Chandra observations of NGC 1569. ApJ 574(2):663-692. https://doi.org/10.1086/341092. arXiv:astro-ph/0203513

Matteucci F (1986) Some considerations on the origin of nitrogen. MNRAS 221:911-921. https://doi.org/ $10.1093 / \mathrm{mnras} / 221.4 .911$

Matteucci F (1996) Galaxy evolution. Fund Cosmic Phys 17:283-396

Matteucci F (2001) The chemical evolution of the galaxy, Astrophysics and Space Science Library, vol 253. Springer, Dordrecht. https://doi.org/10.1007/978-94-010-0967-6

Matteucci F (2012) Chemical evolution of galaxies. Astronomy and Astrophysics Library. Springer, Berlin. https://doi.org/10.1007/978-3-642-22491-1

Matteucci F, Brocato E (1990) Metallicity distribution and abundance ratios in the stars of the galactic bulge. ApJ 365:539. https://doi.org/10.1086/169508

Matteucci F, Chiosi C (1983) Stochastic star formation and chemical evolution of dwarf irregular galaxies. A\&A 123:121-134

Matteucci F, Francois P (1989) Galactic chemical evolution: abundance gradients of individual elements. MNRAS 239:885-904. https://doi.org/10.1093/mnras/239.3.885

Matteucci F, Francois P (1992) Oxygen abundances in halo stars as tests of galaxy formation. A\&A 262(1):L1-L4

Matteucci F, Greggio L (1986) Relative roles of type I and II supernovae in the chemical enrichment of the interstellar gas. A\&A 154(1-2):279-287 
Matteucci F, Recchi S (2001) On the typical timescale for the chemical enrichment from type Ia supernovae in galaxies. ApJ 558(1):351-358. https://doi.org/10.1086/322472. arXiv:astro-ph/ 0105074

Matteucci F, Tornambe A (1985) Carbon deflagrating supernovae and the chemical history of the solar neighbourhood. A\&A 142(1):13-20

Matteucci F, Tosi M (1985) Nitrogen and oxygen evolution in dwarf irregular galaxies. MNRAS 217:391-405. https://doi.org/10.1093/mnras/217.2.391

Matteucci F, Romano D, Molaro P (1999) Light and heavy elements in the galactic bulge. A\&A 341:458-468. arXiv:astro-ph/9810125

Matteucci F, Panagia N, Pipino A et al (2006) A new formulation of the Type Ia supernova rate and its consequences on galactic chemical evolution. MNRAS 372(1):265-275. https://doi.org/10.1111/j. 1365-2966.2006.10848.x. arXiv:astro-ph/0607504

Matteucci F, Spitoni E, Recchi S, Valiante R (2009) The effect of different type Ia supernova progenitors on Galactic chemical evolution. A\&A 501(2):531-538. https://doi.org/10.1051/0004-6361/ 200911869. arXiv:0905.0272 [astro-ph.GA]

Matteucci F, Romano D, Arcones A, Korobkin O, Rosswog S (2014) Europium production: neutron star mergers versus core-collapse supernovae. MNRAS 438(3):2177-2185. https://doi.org/10.1093/ mnras/stt2350. arXiv:1311.6980 [astro-ph.GA]

Matteucci F, Grisoni V, Spitoni E et al (2019) The origin of stellar populations in the Galactic bulge from chemical abundances. MNRAS 487(4):5363-5371. https://doi.org/10.1093/mnras/stz1647. arXiv: 1903.12440 [astro-ph.GA]

Matteucci F, Vasini A, Grisoni V, Schultheis M (2020) Heavy element evolution in the inner regions of the Milky Way. MNRAS 494(4):5534-5541. https://doi.org/10.1093/mnras/staa1118. arXiv:2004. 10133 [astro-ph.GA]

Matteucci F, Molero M, Aguado DS, Romano D (2021) The evolution of lithium: implications of a universal Spite plateau. MNRAS. https://doi.org/10.1093/mnras/stab1234. arXiv:2104.11504 [astroph.GA]

Mayor M, Vigroux L (1981) Effect of the infall of matter on the dynamical structure and chemical evolution of a spiral galaxy. A\&A 98(1):1-8

McKee CF, Parravano A, Hollenbach DJ (2015) Stars, gas, and dark matter in the solar neighborhood. ApJ 814(1):13. https://doi.org/10.1088/0004-637X/814/1/13. arXiv:1509.05334 [astro-ph.GA]

McWilliam A, Rich RM (1994) The first detailed abundance analysis of galactic bulge K giants in Baade's window. ApJS 91:749. https://doi.org/10.1086/191954

Meléndez J, Casagrande L, Ramírez I, Asplund M, Schuster WJ (2010) Observational evidence for a broken Li Spite plateau and mass-dependent Li depletion. A\&A 515:L3. https://doi.org/10.1051/ 0004-6361/200913047. arXiv:1005.2944 [astro-ph.SR]

Meyer BS (1989) Decompression of initially cold neutron star matter: a mechanism for the r-process? ApJ 343:254. https://doi.org/10.1086/167702

Meynet G, Maeder A (2002) Stellar evolution with rotation. VIII. Models at Z $=10^{-5}$ and CNO yields for early galactic evolution. A\&A 390:561-583. https://doi.org/10.1051/0004-6361:20020755. arXiv: astro-ph/0205370

Micali A, Matteucci F, Romano D (2013) The chemical evolution of the Milky Way: the Three Infall Model. MNRAS 436(2):1648-1658. https://doi.org/10.1093/mnras/stt1681. arXiv:1309.1283 [astroph.GA]

Mihos JC, Hernquist L (1994) Star-forming galaxy models: blending star formation into TREESPH. ApJ 437:611. https://doi.org/10.1086/175025

Minchev I, Famaey B, Combes F et al (2011) Radial migration in galactic disks caused by resonance overlap of multiple patterns: self-consistent simulations. A\&A 527:A147. https://doi.org/10.1051/ 0004-6361/201015139. arXiv:1006.0484 [astro-ph.GA]

Minchev I, Chiappini C, Martig M (2013) Chemodynamical evolution of the Milky Way disk. I. The solar vicinity. A\&A 558:A9. https://doi.org/10.1051/0004-6361/201220189. arXiv:1208.1506 [astroph.GA]

Minchev I, Chiappini C, Martig M (2014) Chemodynamical evolution of the Milky Way disk. II. Variations with Galactic radius and height above the disk plane. A\&A 572:A92. https://doi.org/10. 1051/0004-6361/201423487. arXiv:1401.5796 [astro-ph.GA] 
Minniti D, Lucas PW, Emerson JP et al (2010) VISTA Variables in the Via Lactea (VVV): the public ESO near-IR variability survey of the Milky Way. New A 15(5):433-443. https://doi.org/10.1016/j. newast.2009.12.002. arXiv:0912.1056 [astro-ph.GA]

Molaro P, Izzo L, Mason E, Bonifacio P, Della Valle M (2016) Highly enriched ${ }^{7}$ Be in the ejecta of Nova Sagittarii 2015 No. 2 (V5668 Sgr) and the Galactic ${ }^{7}$ Li origin. MNRAS 463(1):L117-L121. https:// doi.org/10.1093/mnrasl/slw169. arXiv:1609.07297 [astro-ph.GA]

Molero M, Simonetti P, Matteucci F, della Valle M (2021) Predicted rates of merging neutron stars in galaxies. MNRAS 500(1):1071-1086. https://doi.org/10.1093/mnras/staa3340. arXiv:2005.10226 [astro-ph.GA]

Moos HW, Sembach KR, Vidal-Madjar A et al (2002) Abundances of deuterium, nitrogen, and oxygen in the local interstellar medium: overview of first results from the FUSE Mission. ApJS 140(1):3-17. https://doi.org/10.1086/339133. arXiv:astro-ph/0112519

Mott A, Spitoni E, Matteucci F (2013) Abundance gradients in spiral discs: is the gradient inversion at high redshift real? MNRAS 435(4):2918-2930. https://doi.org/10.1093/mnras/stt1495. arXiv:1308. 1549 [astro-ph.GA]

Nagashima M, Lacey CG, Baugh CM, Frenk CS, Cole S (2005) The metal enrichment of the intracluster medium in hierarchical galaxy formation models. MNRAS 358(4):1247-1266. https://doi.org/10. 1111/j.1365-2966.2005.08766.x. arXiv:astro-ph/0408529

Nakasato N, Nomoto K (2003) Three-dimensional simulations of the chemical and dynamical evolution of the galactic bulge. ApJ 588(2):842-851. https://doi.org/10.1086/374211. arXiv:astro-ph/0301404

Navarro JF, White SDM (1994) Simulations of dissipative galaxy formation in hierarchically clustering universes-2. Dynamics of the baryonic component in galactic haloes. MNRAS 267(2):401-412. https://doi.org/10.1093/mnras/267.2.401

Ness M, Freeman K (2016) The metallicity distribution of the Milky Way bulge. PASA 33: https://doi. org/10.1017/pasa.2015.51. arXiv:1511.07438 [astro-ph.GA]

Nidever DL, Bovy J, Bird JC et al (2014) Tracing chemical evolution over the extent of the Milky Way's disk with APOGEE Red Clump Stars. ApJ 796(1):38. https://doi.org/10.1088/0004-637X/796/1/38. arXiv:1409.3566 [astro-ph.GA]

Nissen PE, Schuster WJ (1991) uvby- $\beta$ photometry of high-velocity and metal-poor stars. V. Distances, kinematics and ages of halo and disk stars. A\&A 251:457

Nissen PE, Schuster WJ (2010) Two distinct halo populations in the solar neighborhood. Evidence from stellar abundance ratios and kinematics. Astron Astrophys 511:L10. https://doi.org/10.1051/00046361/200913877. arXiv:1002.4514 [astro-ph.GA]

Nissen PE, Christensen-Dalsgaard J, Mosumgaard JR et al (2020) High-precision abundances of elements in solar-type stars. Evidence of two distinct sequences in abundance-age relations. A\&A 640:A81. https://doi.org/10.1051/0004-6361/202038300. arXiv:2006.06013 [astro-ph.SR]

Noguchi M (2018) The formation of solar-neighbourhood stars in two generations separated by 5 billion years. Nature 559(7715):585-588. https://doi.org/10.1038/s41586-018-0329-2. arXiv:1809.02299 [astro-ph.GA]

Nomoto K, Kobayashi C, Tominaga N (2013) Nucleosynthesis in stars and the chemical enrichment of galaxies. ARA\&A 51(1):457-509. https://doi.org/10.1146/annurev-astro-082812-140956

Norris JE, Wyse RFG, Gilmore G et al (2010) Chemical enrichment in the faintest galaxies: the carbon and iron abundance spreads in the Boötes I Dwarf Spheroidal Galaxy and the Segue 1 System. ApJ 723(2):1632-1650. https://doi.org/10.1088/0004-637X/723/2/1632. arXiv:1008.0137 [astro-ph.GA]

O'Meara JM, Burles S, Prochaska JX et al (2006) The deuterium-to-hydrogen abundance ratio toward the QSO SDSS J155810.16-003120.0. ApJ 649(2):L61-L65. https://doi.org/10.1086/508348. arXiv: astro-ph/0608302

Pagel BEJ (1997) Nucleosynthesis and chemical evolution of galaxies. Cambridge University Press, Cambridge

Pagel BEJ, Patchett BE (1975) Metal abundances in nearby stars and the chemical history of the solar neighbourhood. MNRAS 172:13-40. https://doi.org/10.1093/mnras/172.1.13

Palla M, Matteucci F, Spitoni E, Vincenzo F, Grisoni V (2020) Chemical evolution of the Milky Way: constraints on the formation of the thick and thin discs. MNRAS 498(2):1710-1725. https://doi.org/ 10.1093/mnras/staa2437. arXiv:2008.07484 [astro-ph.GA]

Pardi MC, Ferrini F, Matteucci F (1995) Evolution of spiral galaxies. IV. The thick disk in the solar region as an intermediate collapse phase. ApJ 444:207. https://doi.org/10.1086/175596 
Peimbert M, Peimbert A, Luridiana V (2017) A new determination of the primordial helium abundance. Rev Mex Astron Astrofis Conf Ser 49:181

Perryman MAC, de Boer KS, Gilmore G et al (2001) GAIA: composition, formation and evolution of the Galaxy. A\&A 369:339-363. https://doi.org/10.1051/0004-6361:20010085. arXiv:astro-ph/0101235

Pettini M, Zych BJ, Murphy MT, Lewis A, Steidel CC (2008) Deuterium abundance in the most metalpoor damped Lyman alpha system: converging on $\Omega_{b, 0} h^{2}$. MNRAS 391(4):1499-1510. https://doi. org/10.1111/j.1365-2966.2008.13921.x. arXiv:0805.0594 [astro-ph]

Pian E, D’Avanzo P, Benetti S et al (2017) Spectroscopic identification of r-process nucleosynthesis in a double neutron-star merger. Nature 551(7678):67-70. https://doi.org/10.1038/nature24298. arXiv: 1710.05858 [astro-ph.HE]

Pignatari M, Gallino R, Meynet G et al (2008) The s-process in massive stars at low metallicity: the effect of primary ${ }^{14} \mathrm{~N}$ from fast rotating stars. ApJ 687(2):L95. https://doi.org/10.1086/593350. arXiv: 0810.0182 [astro-ph]

Pilkington K, Few CG, Gibson BK et al (2012) Metallicity gradients in disks. Do galaxies form insideout? A\&A 540:A56. https://doi.org/10.1051/0004-6361/201117466. arXiv:1201.6359 [astro-ph.GA]

Pinsonneault MH, Elsworth YP, Tayar J et al (2018) The second APOKASC catalog: the empirical approach. ApJS 239(2):32. https://doi.org/10.3847/1538-4365/aaebfd. arXiv:1804.09983 [astroph.SR]

Polehampton ET, Baluteau JP, Ceccarelli C, Swinyard BM, Caux E (2002) Detection of HD in emission towards Sagittarius B2. A\&A 388:L44-L47. https://doi.org/10.1051/0004-6361:20020630. arXiv: astro-ph/0204432

Portinari L, Chiosi C (2000) On radial gas flows, the galactic bar and chemical evolution in the galactic disc. A\&A 355:929-948. arXiv:astro-ph/0002145

Prantzos N (2003) On the early evolution of the Galactic halo. A\&A 404:211-215. https://doi.org/10. 1051/0004-6361:20030445. arXiv:astro-ph/0303172

Prantzos N (2008a) An introduction to galactic chemical evolution. In: Charbonnel C, Zahn JP (eds) Stellar nucleosynthesis: 50 years after $\mathrm{B}^{2} \mathrm{FH}$. EAS Publications Series, vol 32. EDP Sciences, pp 311-356. https://doi.org/10.1051/eas:0832009. arXiv:0709.0833 [astro-ph]

Prantzos N (2008b) The metallicity distribution of the halo and the satellites of the Milky Way in the hierarchical merging paradigm. A\&A 489(2):525-532. https://doi.org/10.1051/0004-6361: 20079330. arXiv:0807.1502 [astro-ph]

Prantzos N, Boissier S (2000) Chemo-spectrophotometric evolution of spiral galaxies-III. Abundance and colour gradients in discs. MNRAS 313(2):338-346. https://doi.org/10.1046/j.1365-8711.2000. 03228.x. arXiv:astro-ph/9911111

Prantzos N, Casse M, Vangioni-Flam E (1993) Production and evolution of LiBeB isotopes in the galaxy. ApJ 403:630. https://doi.org/10.1086/172233

Prantzos N, de Laverny P, Guiglion G, Recio-Blanco A, Worley CC (2017) The AMBRE project: a study of Li evolution in the Galactic thin and thick discs. A\&A 606:A132. https://doi.org/10.1051/00046361/201731188. arXiv:1709.03998 [astro-ph.GA]

Prantzos N, Abia C, Limongi M, Chieffi A, Cristallo S (2018) Chemical evolution with rotating massive star yields-I. The solar neighbourhood and the s-process elements. MNRAS 476(3):3432-3459. https://doi.org/10.1093/mnras/sty316. arXiv:1802.02824 [astro-ph.GA]

Pritchet CJ, Howell DA, Sullivan M (2008) The progenitors of type Ia supernovae. ApJ 683(1):L25. https://doi.org/10.1086/591314. arXiv:0806.3729 [astro-ph]

Queiroz ABA, Anders F, Chiappini C et al (2020) From the bulge to the outer disc: StarHorse stellar parameters, distances, and extinctions for stars in APOGEE DR16 and other spectroscopic surveys. A\&A 638:A76. https://doi.org/10.1051/0004-6361/201937364. arXiv:1912.09778 [astro-ph.GA]

Rana NC (1991) Chemical evolution of the Galaxy. ARA\&A 29:129-162. https://doi.org/10.1146/ annurev.aa.29.090191.001021

Randich S, Pasquini L, Franciosini E et al (2020) The Gaia-ESO survey: galactic evolution of lithium at high metallicity. A\&A 640:L1. https://doi.org/10.1051/0004-6361/202038688. arXiv:2007.02888 [astro-ph.SR]

Rebolo R, Molaro P, Beckman JE (1988) Lithium abundances inmetal-deficient dwarfs. A\&A 192:192-205

Recchi S, Matteucci F, D'Ercole A (2001) Dynamical and chemical evolution of gas-rich dwarf galaxies. MNRAS 322(4):800-820. https://doi.org/10.1046/j.1365-8711.2001.04189.x. arXiv:astro-ph/ 0002370 
Recchi S, Spitoni E, Matteucci F, Lanfranchi GA (2008) The effect of differential galactic winds on the chemical evolution of galaxies. A\&A 489(2):555-565. https://doi.org/10.1051/0004-6361: 200809879. arXiv:0808.0118 [astro-ph]

Reddy BE, Tomkin J, Lambert DL, Allende Prieto C (2003) The chemical compositions of Galactic disc $\mathrm{F}$ and $\mathrm{G}$ dwarfs. MNRAS 340(1):304-340. https://doi.org/10.1046/j.1365-8711.2003.06305.x. arXiv:astro-ph/0211551

Reddy NA, Steidel CC, Erb DK, Shapley AE, Pettini M (2006) A spectroscopic survey of Redshift $1.4 \lesssim 3.0$ galaxies in the GOODS-North Field: survey description, catalogs, and properties. ApJ 653(2):1004-1026. https://doi.org/10.1086/508851. arXiv:astro-ph/0609296

Renzini A (1994) A hint on the age and helium content of the Galactic Bulge. A\&A 285:L5-L8

Renzini A, Gennaro M, Zoccali M et al (2018) The WFC3 galactic bulge treasury program: relative ages of bulge stars of high and low metallicity. ApJ 863(1):16. https://doi.org/10.3847/1538-4357/ aad09b. arXiv:1806.11556 [astro-ph.GA]

Rich RM (1988) Spectroscopy and abundances of 88 K giants in Baade's window. AJ 95:828. https://doi. org/10.1086/114681

Rich RM, Reitzel DB, Howard CD, Zhao H (2007) The bulge radial velocity assay: techniques and a rotation curve. Astrophys J 658(1):L29-L32. https://doi.org/10.1086/513509. arXiv:astro-ph/ 0611403 [astro-ph]

Rich RM, Origlia L, Valenti E (2012) Detailed abundances for M giants in two inner bulge fields from infrared spectroscopy. ApJ 746(1):59. https://doi.org/10.1088/0004-637X/746/1/59. arXiv:1112. 0306 [astro-ph.GA]

Rizzuti F, Cescutti G, Matteucci F et al (2019) The contribution from rotating massive stars to the enrichment in $\mathrm{Sr}$ and Ba of the Milky Way. MNRAS 489(4):5244-5255. https://doi.org/10.1093/ mnras/stz2505. arXiv:1909.04378 [astro-ph.GA]

Robertson B, Bullock JS, Font AS, Johnston KV, Hernquist L (2005) $\Lambda$ cold dark matter, stellar feedback, and the galactic halo abundance pattern. ApJ 632(2):872-881. https://doi.org/10.1086/452619. arXiv:astro-ph/0501398

Rojas-Arriagada A, Recio-Blanco A, de Laverny P et al (2017) The Gaia-ESO Survey: exploring the complex nature and origins of the Galactic bulge populations. A\&A 601:A140. https://doi.org/10. 1051/0004-6361/201629160. arXiv:1704.03325 [astro-ph.GA]

Romano D, Matteucci F (2003) Nova nucleosynthesis and Galactic evolution of the CNO isotopes. MNRAS 342(1):185-198. https://doi.org/10.1046/j.1365-8711.2003.06526.x. arXiv:astro-ph/ 0302233

Romano D, Tosi M, Matteucci F, Chiappini C (2003) Light element evolution resulting from WMAP data. MNRAS 346(1):295-303. https://doi.org/10.1046/j.1365-2966.2003.07083.x. arXiv:astro-ph/ 0308181

Romano D, Karakas AI, Tosi M, Matteucci F (2010) Quantifying the uncertainties of chemical evolution studies. II. Stellar yields. A\&A 522:A32. https://doi.org/10.1051/0004-6361/201014483. arXiv: 1006.5863 [astro-ph.GA]

Romano D, Franchini M, Grisoni V et al (2020) The variation of carbon abundance in galaxies and its implications. A\&A 639:A37. https://doi.org/10.1051/0004-6361/202037972. arXiv:2005.05717 [astro-ph.GA]

Rosswog S, Liebendörfer M, Thielemann FK et al (1999) Mass ejection in neutron star mergers. A\&A 341:499-526. arXiv:astro-ph/9811367

Roškar R, Debattista VP, Quinn TR, Stinson GS, Wadsley J (2008) Riding the spiral waves: implications of stellar migration for the properties of galactic disks. ApJ 684(2):L79. https://doi.org/10.1086/ 592231. arXiv:0808.0206 [astro-ph]

Ruiter AJ (2020) Type Ia supernova sub-classes and progenitor origin. IAU Symp 357:1-15. https://doi. org/10.1017/S1743921320000587. arXiv:2001.02947 [astro-ph.SR]

Ryan SG, Norris JE (1991) Subdwarf studies. III. The halo metallicity distribution. AJ 101:1865-1878. https://doi.org/10.1086/115812

Ryde N, Schultheis M, Grieco V et al (2016) Chemical evolution of the inner 2 degrees of the Milky Way bulge: $[\alpha / \mathrm{Fe}]$ trends and metallicity gradients. AJ 151(1):1. https://doi.org/10.3847/0004-6256/151/ 1/1. arXiv:1510.02622 [astro-ph.GA]

Salpeter EE (1955) The luminosity function and stellar evolution. ApJ 121:161. https://doi.org/10.1086/ 145971

Samland M, Gerhard OE (2003) The formation of a disk galaxy within a growing dark halo. A\&A 399:961-982. https://doi.org/10.1051/0004-6361:20021842. arXiv:astro-ph/0301499 
Sbordone L, Bonifacio P, Caffau E et al (2010) The metal-poor end of the Spite plateau. I. Stellar parameters, metallicities, and lithium abundances. A\&A 522:A26. https://doi.org/10.1051/00046361/200913282. arXiv:1003.4510 [astro-ph.GA]

Scalo JM (1986) The stellar initial mass function. Fund Cosmic Phys 11:1-278

Schmidt M (1959) The rate of star formation. ApJ 129:243. https://doi.org/10.1086/146614

Schmidt M (1963) The rate of star formation. II. The rate of formation of stars of different mass. ApJ 137:758. https://doi.org/10.1086/147553

Schönrich R, Binney J (2009) Chemical evolution with radial mixing. MNRAS 396(1):203-222. https:// doi.org/10.1111/j.1365-2966.2009.14750.x. arXiv:0809.3006 [astro-ph]

Schönrich RA, Weinberg DH (2019) The chemical evolution of r-process elements from neutron star mergers: the role of a 2-phase interstellar medium. MNRAS 487(1):580-594. https://doi.org/10. 1093/mnras/stz1126. arXiv:1901.09938 [astro-ph.GA]

Schörck T, Christlieb N, Cohen JG et al (2009) The stellar content of the Hamburg/ESO survey. V. The metallicity distribution function of the Galactic halo. A\&A 507(2):817-832. https://doi.org/10.1051/ 0004-6361/200810925. arXiv:0809.1172 [astro-ph]

Schultheis M, Rojas-Arriagada A, García Pérez AE et al (2017) Baade's window and APOGEE. Metallicities, ages, and chemical abundances. A\&A 600:A14. https://doi.org/10.1051/0004-6361/ 201630154. arXiv:1702.01547 [astro-ph.GA]

Searle L, Zinn R (1978) Composition of halo clusters and the formation of the galactic halo. ApJ 225:357-379. https://doi.org/10.1086/156499

Sharma S, Hayden MR, Bland-Hawthorn J (2020) Chemical enrichment and radial migration in the galactic disk-the origin of the $[\alpha / \mathrm{Fe}]$ double sequence. arXiv:2005.03646 [astro-ph.GA]

Shen S, Cooke RJ, Ramirez-Ruiz E et al (2015) The history of r-process enrichment in the Milky Way. ApJ 807(2):115. https://doi.org/10.1088/0004-637X/807/2/115. arXiv:1407.3796 [astro-ph.GA]

Shore SN, De Gennaro AI (2020) NLTE modeling and spectroscopically derived abundances of lithium and beryllium for classical nova ejecta. A\&A 639:L12. https://doi.org/10.1051/0004-6361/ 202038599

Silva Aguirre V, Bojsen-Hansen M, Slumstrup D et al (2018) Confirming chemical clocks: asteroseismic age dissection of the Milky Way disc(s). MNRAS 475(4):5487-5500. https://doi.org/10.1093/mnras/ sty150. arXiv:1710.09847 [astro-ph.GA]

Simion IT, Belokurov V, Koposov SE (2019) Common origin for Hercules-Aquila and Virgo Clouds in Gaia DR2. MNRAS 482(1):921-928. https://doi.org/10.1093/mnras/sty2744. arXiv:1807.01335 [astro-ph.GA]

Simonetti P, Matteucci F, Greggio L, Cescutti G (2019) A new delay time distribution for merging neutron stars tested against Galactic and cosmic data. MNRAS 486(2):2896-2909. https://doi.org/ 10.1093/mnras/stz991. arXiv:1901.02732 [astro-ph.HE]

Snaith ON, Haywood M, Di Matteo P et al (2014) The dominant epoch of star formation in the Milky Way formed the thick disk. ApJ 781(2):L31. https://doi.org/10.1088/2041-8205/781/2/L31. arXiv: 1401.1835 [astro-ph.GA]

Sneden C, Lambert DL, Whitaker RW (1979) The oxygen abundance in metal-poor stars. ApJ 234:964-972. https://doi.org/10.1086/157580

Spergel DN, Verde L, Peiris HV et al (2003) First-year Wilkinson Microwave Anisotropy Probe (WMAP) observations: determination of cosmological parameters. ApJS 148(1):175-194. https://doi.org/10. 1086/377226. arXiv:astro-ph/0302209

Spite F, Spite M (1982) Abundance of lithium in unevolved stars and old disk stars: interpretation and consequences. A\&A 115:357-366

Spite F, Spite M (1986) Lithium abundance in the nitrogen-rich halo dwarfs. A\&A 163:140-144

Spitoni E, Matteucci F (2011) Effects of the radial flows on the chemical evolution of the Milky Way disk. A\&A 531:A72. https://doi.org/10.1051/0004-6361/201015749. arXiv:1104.4881 [astroph.GA]

Spitoni E, Romano D, Matteucci F, Ciotti L (2015) The effect of stellar migration on galactic chemical evolution: a heuristic approach. ApJ 802(2):129. https://doi.org/10.1088/0004-637X/802/2/129. arXiv:1407.5797 [astro-ph.GA]

Spitoni E, Vincenzo F, Matteucci F, Romano D (2016) Are ancient dwarf satellites the building blocks of the Galactic halo? MNRAS 458(3):2541-2552. https://doi.org/10.1093/mnras/stw519. arXiv:1603. 00344 [astro-ph.GA] 
Spitoni E, Vincenzo F, Matteucci F (2017) New analytical solutions for chemical evolution models: characterizing the population of star-forming and passive galaxies. A\&A 599:A6. https://doi.org/10. 1051/0004-6361/201629745. arXiv:1605.05603 [astro-ph.GA]

Spitoni E, Silva Aguirre V, Matteucci F, Calura F, Grisoni V (2019) Galactic archaeology with asteroseismic ages: evidence for delayed gas infall in the formation of the Milky Way disc. A\&A 623:A60. https://doi.org/10.1051/0004-6361/201834188. arXiv:1809.00914 [astro-ph.GA]

Spitoni E, Verma K, Silva Aguirre V, Calura F (2020) Galactic archaeology with asteroseismic ages. II. Confirmation of a delayed gas infall using Bayesian analysis based on MCMC methods. A\&A 635:A58. https://doi.org/10.1051/0004-6361/201937275. arXiv:1912.04312 [astro-ph.GA]

Spitoni E, Verma K, Silva Aguirre V et al (2021) APOGEE DR16: a multi-zone chemical evolution model for the Galactic disc based on MCMC methods. A\&A 647:A73. https://doi.org/10.1051/00046361/202039864. arXiv:2101.08803 [astro-ph.GA]

Springel V (2005) The cosmological simulation code GADGET-2. MNRAS 364(4):1105-1134. https:// doi.org/10.1111/j.1365-2966.2005.09655.x. arXiv:astro-ph/0505010

Springel V, Hernquist L (2003) Cosmological smoothed particle hydrodynamics simulations: a hybrid multiphase model for star formation. MNRAS 339(2):289-311. https://doi.org/10.1046/j.1365-8711. 2003.06206.x. arXiv:astro-ph/0206393

Stahler SW, Palla F (2004) The formation of stars. Wiley-VCH, Weinheim

Starrfield S, Bose M, Iliadis C et al (2020) Carbon-oxygen classical novae are galactic ${ }^{7}$ Li producers as well as potential supernova Ia progenitors. ApJ 895(1):70. https://doi.org/10.3847/1538-4357/ ab8d23. arXiv:1910.00575 [astro-ph.SR]

Steigman G, Tosi M (1992) Galactic evolution of D and 3He. ApJ 401:150. https://doi.org/10.1086/ 172048

Steinmetz M, Mueller E (1994) The formation of disk galaxies in a cosmological context: populations, metallicities and metallicity gradients. A\&A 281(3):L97-L100. arXiv:astro-ph/9312010

Strolger LG, Riess AG, Dahlen T et al (2004) The Hubble Higher $z$ Supernova Search: supernovae to $z \sim 1.6$ and constraints on Type Ia progenitor models. ApJ 613(1):200-223. https://doi.org/10.1086/ 422901. arXiv:astro-ph/0406546

Suzuki TK, Yoshii Y (2001) A new model for the evolution of light elements in an inhomogeneous galactic halo. ApJ 549(1):303-319. https://doi.org/10.1086/319049. arXiv:astro-ph/0010108

Tajitsu A, Sadakane K, Naito H, Arai A, Aoki W (2015) Explosive lithium production in the classical nova V339 Del (Nova Delphini 2013). Nature 518(7539):381-384. https://doi.org/10.1038/ nature14161. arXiv:1502.05598 [astro-ph.SR]

Tajitsu A, Sadakane K, Naito H et al (2016) The ${ }^{7}$ Be II resonance lines in two classical novae V5668 Sgr and V2944 Oph. ApJ 818(2):191. https://doi.org/10.3847/0004-637X/818/2/191. arXiv:1601.05168 [astro-ph.SR]

Tanvir NR, Levan AJ, González-Fernández C et al (2017) The emergence of a lanthanide-rich kilonova following the merger of two neutron stars. ApJ 848(2):L27. https://doi.org/10.3847/2041-8213/ aa90b6. arXiv:1710.05455 [astro-ph.HE]

Thielemann FK, Isern J, Perego A, von Ballmoos P (2018) Nucleosynthesis in supernovae. Space Sci Rev 214(3):62. https://doi.org/10.1007/s11214-018-0494-5

Thomas D (1999) Abundance ratios in hierarchical galaxy formation. MNRAS 306(3):655-661. https:// doi.org/10.1046/j.1365-8711.1999.02552.x. arXiv:astro-ph/9901226

Thomas D, Kauffmann G (1999) Probing star formation timescales in elliptical galaxies. In: Hubeny I, Heap S, Cornett R (eds) Spectrophotometric dating of stars and galaxies. ASP conference series, vol 192. Astronomical Society of the Pacific, p 261. arXiv:astro-ph/9906216

Tinsley BM (1980) Evolution of the stars and gas in galaxies. Fund Cosmic Phys 5:287-388

Tolstoy E, Hill V, Tosi M (2009) Star-formation histories, abundances, and kinematics of dwarf galaxies in the local group. ARA\&A 47(1):371-425. https://doi.org/10.1146/annurev-astro-082708-101650. arXiv:0904.4505 [astro-ph.CO]

Tornatore L, Borgani S, Dolag K, Matteucci F (2007) Chemical enrichment of galaxy clusters from hydrodynamical simulations. MNRAS 382(3):1050-1072. https://doi.org/10.1111/j.1365-2966. 2007.12070.x. arXiv:0705.1921 [astro-ph]

Tosi M (1982) CNO isotopes and galactic chemical evolution. ApJ 254:699-707. https://doi.org/10.1086/ 159782

Tosi M (1988a) Models of galactic chemical evolution-the problem of uniqueness. A\&A 197(1-2):33-46

Tosi M (1988b) The effect of metal-rich infall on galactic chemical evolution. A\&A 197(1-2):47-51 
Totani T, Morokuma T, Oda T, Doi M, Yasuda N (2008) Delay time distribution measurement of type Ia Supernovae by the Subaru/XMM-Newton Deep Survey and implications for the progenitor. PASJ 60:1327. https://doi.org/10.1093/pasj/60.6.1327. arXiv:0804.0909 [astro-ph]

Travaglio C, Galli D, Gallino R et al (1999) Galactic chemical evolution of heavy elements: from Barium to Europium. ApJ 521(2):691-702. https://doi.org/10.1086/307571. arXiv:astro-ph/9903451

Travaglio C, Burkert A, Galli D (2001) Inhomogeneous chemical evolution of the Galactic halo. Nucl Phys A 688(1-2):396-398. https://doi.org/10.1016/S0375-9474(01)00735-7

Troja E, Piro L, van Eerten $\mathrm{H}$ et al (2017) The X-ray counterpart to the gravitational-wave event GW170817. Nature 551(7678):71-74. https://doi.org/10.1038/nature24290. arXiv:1710.05433 [astro-ph.HE]

Vader JP (1986) Metal-enhanced galactic winds. I. ApJ 305:669. https://doi.org/10.1086/164281

Valenti E, Zoccali M, Renzini A et al (2013) Stellar ages through the corners of the boxy bulge. A\&A 559:A98. https://doi.org/10.1051/0004-6361/201321962. arXiv:1309.4570 [astro-ph.GA]

van de Voort F, Quataert E, Hopkins PF, Kereš D, Faucher-Giguère CA (2015) Galactic r-process enrichment by neutron star mergers in cosmological simulations of a Milky Way-mass galaxy. MNRAS 447(1):140-148. https://doi.org/10.1093/mnras/stu2404. arXiv:1407.7039 [astro-ph.GA]

van den Hoek LB, Groenewegen MAT (1997) New theoretical yields of intermediate mass stars. A\&AS 123:305-328. https://doi.org/10.1051/aas:1997162

Vidal-Madjar A, Lemoine M, Ferlet R et al (1998) Detection of spatial variations in the (D/H) ratio in the local interstellar medium. A\&A 338:694-712. arXiv:astro-ph/9807004

Vincenzo F, Kobayashi C (2020) Stellar migrations and metal flows-chemical evolution of the thin disc of a simulated Milky Way analogous galaxy. MNRAS 496(1):80-94. https://doi.org/10.1093/mnras/ staa1451. arXiv:2004.08050 [astro-ph.GA]

Vincenzo F, Matteucci F, Vattakunnel S, Lanfranchi GA (2014) Chemical evolution of classical and ultra-faint dwarf spheroidal galaxies. MNRAS 441(4):2815-2830. https://doi.org/10.1093/mnras/ stu710. arXiv:1404.2476 [astro-ph.GA]

Vincenzo F, Matteucci F, Belfiore F, Maiolino R (2016) Modern yields per stellar generation: the effect of the IMF. MNRAS 455(4):4183-4190. https://doi.org/10.1093/mnras/stv2598. arXiv: 1503.08300 [astro-ph.GA]

Vincenzo F, Spitoni E, Calura F et al (2019) The fall of a giant. Chemical evolution of Enceladus, alias the Gaia Sausage. MNRAS 487(1):L47-L52. https://doi.org/10.1093/mnrasl/slz070. arXiv:1903. 03465 [astro-ph.GA]

Wanajo S, Kajino T, Mathews GJ, Otsuki K (2001) The r-process in neutrino-driven winds from nascent, "Compact" neutron stars of core-collapse supernovae. ApJ 554(1):578-586. https://doi.org/10.1086/ 321339. arXiv:astro-ph/0102261

Wehmeyer B, Fröhlich C, Pignatari M, Thielemann FK (2019) Inhomogeneous chemical evolution of r-process elements in the galactic halo. In: Formicola A, Junker M, Gialanella L, Imbriani G (eds) Nuclei in the cosmos XV. Springer Proceedings in Physics, vol 219. Springer, Cham, pp 91-96. https://doi.org/10.1007/978-3-030-13876-9_15

Whelan J, Icko Iben J (1973) Binaries and Supernovae of Type I. ApJ 186:1007-1014. https://doi.org/10. $1086 / 152565$

White SDM, Frenk CS (1991) Galaxy formation through hierarchical clustering. ApJ 379:52. https://doi. org/10.1086/170483

Winteler C, Käppeli R, Perego A et al (2012) Magnetorotationally driven supernovae as the origin of early galaxy r-process elements? ApJ 750(1):L22. https://doi.org/10.1088/2041-8205/750/1/L22. arXiv:1203.0616 [astro-ph.SR]

Woosley SE, Weaver TA (1995) The evolution and explosion of massive stars. II. Explosive hydrodynamics and nucleosynthesis. ApJS 101:181. https://doi.org/10.1086/192237

Woosley SE, Hartmann DH, Hoffman RD, Haxton WC (1990) The v-process. ApJ 356:272. https://doi. org/10.1086/168839

Woosley SE, Wilson JR, Mathews GJ, Hoffman RD, Meyer BS (1994) The r-process and neutrino-heated Supernova Ejecta. ApJ 433:229. https://doi.org/10.1086/174638

Wyse RFG, Gilmore G (1992) Formation and evolution of the galactic bulge and spheroid: where did the spheroid gas go? AJ 104:144. https://doi.org/10.1086/116226

Yoon SC, Woosley SE, Langer N (2010) Type Ib/c supernovae in binary systems I evolution and properties of the progenitor stars. ApJ 725(1):940-954. https://doi.org/10.1088/0004-637X/725/1/ 940. arXiv:1004.0843 [astro-ph.SR] 
Yoshii Y (1982) Density distribution of faint stars in the direction of the north galactic pole. PASJ 34:365-379

Yuan H, Liu X, Xiang M et al (2015) Stellar loci II. A model-free estimate of the binary fraction for field FGK stars. ApJ 799(2):135. https://doi.org/10.1088/0004-637X/799/2/135. arXiv:1412.1233 [astroph.SR]

Zasowski G, Schultheis M, Hasselquist S et al (2019) APOGEE DR14/DR15 abundances in the inner Milky Way. ApJ 870(2):138. https://doi.org/10.3847/1538-4357/aaeff4. arXiv:1811.01097 [astroph.GA]

Zoccali M, Gonzalez OA, Vasquez S et al (2014) The GIRAFFE Inner Bulge Survey (GIBS). I. Survey description and a kinematical map of the Milky Way bulge. A\&A 562:A66. https://doi.org/10.1051/ 0004-6361/201323120. arXiv:1401.4878 [astro-ph.GA]

Zoccali M, Vasquez S, Gonzalez OA et al (2017) The GIRAFFE Inner Bulge Survey (GIBS). III. Metallicity distributions and kinematics of 26 Galactic bulge fields. A\&A 599:A12. https://oi.org/ $10.1051 / 0004-6361 / 201629805$

Publisher's Note Springer Nature remains neutral with regard to jurisdictional claims in published maps and institutional affiliations. 